\title{
A STUDY OF SEMI-AUTOMATED TRACING
}

\author{
A Thesis \\ Presented to \\ the Faculty of California Polytechnic State University \\ San Luis Obispo
}

\author{
In Partial Fulfillment \\ of the Requirements for the Degree \\ Master of Science in Computer Science
}

by

Jeffrey Holden

June 2011 
(C) 2011

Jeffrey Holden

ALL RIGHTS RESERVED 


\section{COMMITTEE MEMBERSHIP}

TITLE:

AUTHOR:

DATE SUBMITTED:
A Study of Semi-Automated Tracing

Jeffrey Holden

June 2011

COMMITTEE CHAIR: Dr. Alex Dekhtyar

COMmitTeE MEMBER: Dr. Clark Savage Turner Esq.

COMMITTEE MEMBER: Dr. Gene Fisher 


\begin{abstract}
A Study of Semi-Automated Tracing

by

Jeffrey Holden
\end{abstract}

Requirements tracing is crucial for software engineering practices including change analysis, regression testing, and reverse engineering. The requirements tracing process produces a requirements traceability matrix(TM) which links high- and low-level document elements. Manually generating a TM is laborious, time consuming, and error-prone. Due to these challenges TMs are often neglected. Automated information retrieval(IR) techniques are used with some efficiency. However, in mission- or safety-critical systems a human analyst is required to vet the candidate TM. This introduces semi-automated requirements tracing, where IR methods present a candidate TM and a human analyst validates it, producing a final TM. In semi-automated tracing the focus becomes the quality of the final TM. This thesis expands upon the research of Cuddeback et al. by examining how human analysts interact with candidate TMs. We conduct two experiments, one using an automated tracing tool and the other using manual validation. We conduct formal statistical analysis to determine the key factors impacting the analyst's tracing performance. Additionally, we conduct a pilot study investigating how analysts interact with TMs generated by automated IR methods. Our research statistically confirms the finding of Cuddeback et al. that the strongest impact on analyst performance is the initial TM quality. Finally we show evidence that applying local filters to IR results produce the best candidate TMs. 


\section{Acknowledgements}

This work is funded in part by a grant from Lockheed Martin Corp. The author would like to thank John Dalbey for providing the Changestyle dataset and Jane Huang for use of the HIPAA dataset. In addition I would like to thank Clark Turner, Gene Fisher, Phillip Nico, John Dalbey and Jane Hayes for allowing us to run experiments in their classes. Additionally I would like to thank Olga Dekhtyar for her assistance on the statistical analysis of our experiment's results. Finally I would like to thank my advisor, Alex Dekhtyar, for all his guidance and countless hours of support throughout this project. This thesis would not have turned out nearly as well without his insight. 


\section{Contents}

List of Tables $\quad$ X

List of Figures xii

1 Introduction 1

2 Background and Related Work 11

2.1 Automated Requirements Tracing . . . . . . . . . . . . . . . 12

2.2 Information Retrieval Techniques . . . . . . . . . . . . . . . 13

2.2.1 Vector Space Model . . . . . . . . . . . . . . . . . . . . 15

2.3 Measures. . . . . . . . . . . . . . . . . . 17

2.4 The Human Side of Automated Traceability . . . . . . . . . . . . 21

2.5 Traceability tools . . . . . . . . . . . . . . . 27

3 Overview of Experiments $\quad 29$

4 Experimental Design using Artificially Generated TMs 33

4.1 Experimental Tools . . . . . . . . . . . . . . . . 35

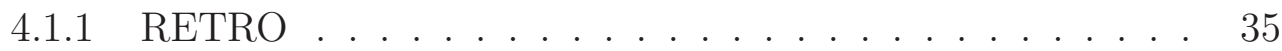

4.1.2 RETRO.net tool .................. 37

4.2 Experimental Design . . . . . . . . . . . . . . . . . 40

4.2.1 Manual study . . . . . . . . . . . . . . . . . . . 42

4.2.2 RETRO.net study . . . . . . . . . . . . . . . 43

4.3 What we Traced . . . . . . . . . . . . . . 45

4.3.1 Training Dataset ............... 45

4.3.2 ChangeStyle Dataset . . . . . . . . . . . . . . . . 45

4.4 Information Collected . . . . . . . . . . . . . . . . 47 
4.4.1 Baseline Independent Variables . . . . . . . . . . . . . . . 49

4.4 .2 Observed Independent Variables . . . . . . . . . . . . . . . 49

4.4 .3 Dependent Variables . . . . . . . . . . . . . . . . . . 51

4.4.4 Research Questions ................ 52

5 Results for Artificially Generated TM experiment 54

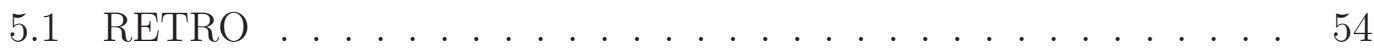

5.2 RETROnet . . . . . . . . . . . . . . . . 55

5.3 Manual study . . . . . . . . . . . . . . . . . . 55

5.4 Observation Analysis . . . . . . . . . . . . . . 56

5.4.1 Movement towards recall $=$ precision . . . . . . . . . . 57

5.4.2 Regional Behaviors . . . . . . . . . . . . . . 59

5.4.3 Who improved Accuracy . . . . . . . . . . . 67

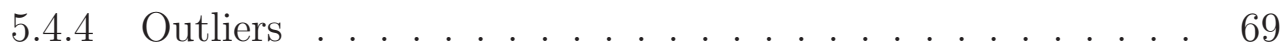

5.5 Statistical Analysis . . . . . . . . . . . . . . . 71

5.5.1 Baseline independent variables . . . . . . . . . . . 71

5.5.2 Observed Independent Variables . . . . . . . . . . . 73

5.6 Summary of Key Findings . . . . . . . . . . . . . . . . 76

6 Naturally Generated TM Experiment Design $\quad 78$

6.1 Software tools used . . . . . . . . . . . . . . . . . . 79

6.1.1 Filtering techniques . . . . . . . . . . . . . 79

6.1 .2 User Interfaces . . . . . . . . . . . . . . . 80

6.2 Pilot Study Experimental design . . . . . . . . . . . . . . 82

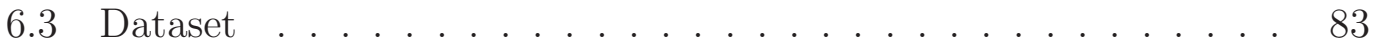

6.3.1 HIPAA-ClearHealth Dataset . . . . . . . . . . . . 83

6.4 Information collected . . . . . . . . . . . . . 84

6.5 Research questions . . . . . . . . . . . . . . 86

7 Naturally Generated TM Experiment Results $\quad 87$

7.1 ChangeStyle ...................... 87

7.2 HIPAA . . . . . . . . . . . . . . . . . . . . . . . 88

7.3 IR method Used . . . . . . . . . . . . . . . . . . . . . . . 89 
7.4 Filtering Method Used . . . . . . . . . . . . . . . . 91

7.5 ChangeStyle vs HIPAA Analyst Behavior . . . . . . . . . . . . . . 98

8 Future Work 103

9 Conclusions $\quad 105$

$\begin{array}{ll}\text { Bibliography } & 108\end{array}$

A Informed Consent Form 113

B Manual Trace Instructions 118

C RETRO.net Tool Instructions 120

D Experiment Instructions 123

$\begin{array}{ll}\text { E Pre-Study Survey } & 126\end{array}$

$\begin{array}{llr}\text { F Post-Study Survey } & 129\end{array}$

G Training Dataset 132

G.1 Functional Requirements . . . . . . . . . . . . . . . . . . . . 132

G.2 System Tests . . . . . . . . . . . . . . . . 135

H Experimental Dataset $\quad 137$

H.1 Requirements . . . . . . . . . . . . . . . . . . . . 137

H.2 System Tests . . . . . . . . . . . . . . . . . . . . . . . . . . . 143

I HIPAA Requirements 152

I.1 Requirements . . . . . . . . . . . . . . . . . . 152 


\section{List of Tables}

2.1 Summary of research in IR techniques for automated traceability $[6] \ldots \ldots \ldots \ldots \ldots \ldots \ldots \ldots$

2.2 Acceptable levels for recall, precision, and lag. . . . . . . . . 21

4.1 Summary of experimental cohorts . . . . . . . . . . . . . . 33

4.2 Baseline Independent Variables . . . . . . . . . . . . . . . 48

4.3 Observed Independent Variables . . . . . . . . . . . . . . . . . 48

4.4 Response (dependent) variables . . . . . . . . . . . . . . . 48

4.5 Response (dependent) variables . . . . . . . . . . . . . . . . . 49

5.1 Influence of initial precision and initial recall on response variables (degrees of freedom: 2, 81) . . . . . . . . . . . . 71

5.2 Influence of initial $f_{2}$-measure on response variables (degrees of freedom: 1,82$) \ldots \ldots \ldots \ldots \ldots \ldots \ldots \ldots \ldots$

5.3 Influence of Starting Quadrant on Response Variables (degrees of freedom: 3,80$) \ldots \ldots \ldots \ldots \ldots \ldots \ldots$

5.4 Analysis for observed independent variables controlling for initial TM precision and recall . . . . . . . . . . . . . . 74

5.5 Influence of ValidEff on response variables $\ldots \ldots \ldots \ldots$

6.1 ChangeStyle Pilot Study Experimental Design . . . . . . . . . . 82

6.2 HIPAA Pilot Study Experimental Design . . . . . . . . . . . . 83

6.3 Baseline Independent Variables . . . . . . . . . . . . . . 85

7.1 Overview of our natural TM generation experiments . . . . . . 87 
7.2 Performance for analysts using naturally generated TMs vetting the ChangeStyle dataset . . . . . . . . . . . . . . . . . . 88

7.3 Performance for analysts using naturally generated TMs vetting the HIPAA dataset . . . . . . . . . . . . . . . . . . . . 89

7.4 Comparing analyst performance when using TF-IDF and Okapi generated TMs while vetting the ChangeStyle dataset . . . . . . 90 


\section{List of Figures}

1.1 The overall process of a) manual tracing b) fully automated tracing c) semi-automated tracing. . . . . . . . . . . . . . . . . 4

1.2 Average precision and recall drift from [6], separated in four quad-

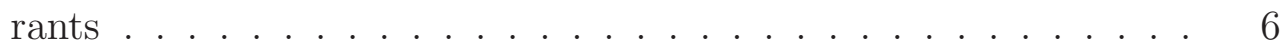

2.1 The tracing process. . . . . . . . . . . . . . . 14

2.2 Results from a pilot study. . . . . . . . . . . . . . . . . 22

2.3 Change in precision and recall for participants from the Cuddeback et al. study in the four regions of the precision-recall space[6]. . . 23

4.1 Overview of our research expanding upon the results of Cuddeback et al. . . . . . . . . . . . . . . . . . . . . . 34

4.2 Overview of the RETRO UI (a) shows the trace tab where (b) shows the free browse mode. . . . . . . . . . . . . . . 36

4.3 RETRO.net's UI (a) shows the recommended links tab where (b) shows the free browse, all links mode. Also see figure 4.4 for the keyword search view. . . . . . . . . . . . . . . . . . . . 38

4.4 RETRO.net's keyword tab, allowing analysts to search low or highlevel elements by keywords. . . . . . . . . . . . . . . . . 39

4.5 Submission instructions for participants using RETRO.net . . . . 44

5.1 Starting TM in precision and recall space for (a) Cuddeback et al., (b) RETRO.net, (c) manual, and (d) all studies . . . . . . . . . 56

5.2 Change in precision and recall from initial to final TMs for the (a) Cuddeback, (b) RETRO.net, (c) manual, and (d) all three studies

5.3 Change in the size of the TM from initial to file for the (a) Cuddeback, (b) manual, (c) RETRO.net, and (d) all three studies . . 60 
5.4 Average analyst performance for each of four regions in the (a) Cuddeback, (b) manual, (c) RETRO.net, and (d) all three studies

5.5 Analyst performance for each of four regions in the RETRO study: (a) Higha-precision, Low-recall; (b) High-precision, High-recall; (c) Low-precision, Low-Recall; and (d) Low-precsion, High-recall . . . 62

5.6 Analyst performance for each of four regions in the manual study: (a) Higha-precision, Low-recall; (b) High-precision, High-recall; (c) Low-precision, Low-Recall; and (d) Low-precsion, High-recall . . .

5.7 Analyst performance for each of four regions in the RETRO.net study: (a) Higha-precision, Low-recall; (b) High-precision, Highrecall; (c) Low-precision, Low-Recall; and (d) Low-precsion, Highrecall . . . . . . . . . . . . . . . . . . . . . 64

5.8 Change in $f_{2}$-measure for the (a) RETRO, (b) manual, (c) RETRO.net, and $(\mathrm{d})$ all studies combined. . . . . . . . . . . . . 68

5.9 Inital $f_{2}$-measure vs. Final $f_{2}$-measure for the (a) RETRO, (b) manual, (c) RETRO.net, and (d) all studies combined. . . . . . . 69

5.10 Analyst performance for (a) outliers, (b) analysts vetting the largest TMs, (c) Positive region in high precision, low recall area, and (d) border region between low and high precision with high recall. . . 70

5.11 Participants with ValidEff values of 4 or 5 . . . . . . . . . 75

6.1 Initial TM accuracy of (a) ChangeStyle, (b) HIPAA, and (c) both datasets . . . . . . . . . . . . . . 84

7.1 Analyst performance when using naturally generated TMs and the ChangeStyle dataset . . . . . . . . . . . . . . . . . . . . . . . 89

7.2 Analyst performance when using naturally generated TMs and the

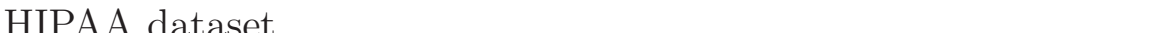

7.3 Comparing analyst performance based on IR method differences. (a) shows analysts using TF-IDF and (b) shows analysts using Okapi.

7.4 Comparing analyst performance based on filtering technique. (a) and (c) show analyst performance using no filtering, and (b) and 7.4(d) show analyst performance using value filtering. . . . . . . .

7.5 Comparing analyst performance based on filtering technique. (a) and (c) show analyst performance using local top one filtering, and (b) and 7.5(d) show analyst performance using local top three filtering. . . . . . . . . . . . . . . . . . . . . . . 94 
7.6 Comparing analyst performance based on filtering technique. (a) and (c) show analyst performance using global top 23 filtering, and (b) and 7.6(d) show analyst performance using global top 96 filtering. . . . . . . . . . . . . . . . . . 97

7.7 Comparing analyst performance when vetting HIPAA based on filtering technique. (a) and (c) show analyst performance using no filtering, and (b) and 7.7(d) show analyst performance using value filtering. . . . . . . . . . . . . . . . . . . . . 99

7.8 Comparing analyst performance when vetting HIPAA based on filtering technique. (a) and (c) show analyst performance using local top one filtering, and (b) and 7.8(d) show analyst performance using local top three filtering. . . . . . . . . . . . . . . 102 


\section{Chapter 1}

\section{Introduction}

Many common software engineering practices including change analysis, regression testing, and reverse engineering have a common ground: a traceability matrix (TM) is required to preform these tasks efficiently. A TM is generated through the process of requirements tracing which, according to Gotel et al., is the process of following "the life of a requirement, in both a forwards and backwards direction (i.e., from its origins, through its development and specification, to its subsequent deployment and use, and through all periods of on-going refinement and iteration in any of these phases)" [14]. The traceability matrix specifies the links between artifacts of the software process, for example determining which requirements are addressed by which test cases for a software system.

There is little doubt that most, if not all, complex software projects could benefit greatly from the creation and maintenance of TMs. Unfortunately, due to project priorities as well as the laborious, time consuming, and error-prone nature of the tracing task, TMs are often neglected. When TMs are created and maintained, the process tends to be manual, requiring a human analyst to evaluate all potential links between the documents. In many mission- and/or 
safety-critical systems, these TMs are vital and are frequently mandated by government regulations. However, even though TMs are desirable or required, they frequently receive a lower priority.

Due to the vast number of software engineering tasks where a TM is beneficial, and the fact that generating TMs can be an arduous and error-prone task, researchers have looked for ways to automate the TM creation process. Current research has focused on applying techniques from the field of information retrieval. Information retrieval techniques are concerned with which documents from a collection are relevant to particular queries. In automated requirements traceability the requirements act as queries and the elements of the low-level artifact serve as the document collection being searched [17].

To date, most research has shown that information retrieval techniques can be applied to successfully generate candidate TMs in an automated fashion [2, 25, 16]. In this document "candidate TM" refers to a TM that has not been vetted by a human analyst. Current automated methods can recover a large portion of the valid links, however they also produce many false positives. The majority of current research has focused on this area, attempting to get the automated methods to eliminate false positives while still returning all valid links, getting closer to the so called "golden standard" [7]. In traceability research, the quality of an automated method has been equated to the overall accuracy of the result produced: a method producing more accurate candidate TMs is considered preferable to a method producing less accurate candidate TMs.

With the advent of automated techniques, two different tracing processes are possible: manual and fully automated tracing. The tracing process can be broken down into two parts: (1) collection of potential links and (2) validation of the links. In manual tracing, both parts 1 and 2 are accomplished by a human 
analyst. In a fully automated tracing process, parts 1 and 2 are both done by special purpose tracing software.

However, in mission- and safety-critical systems, TMs are often required to be vetted by a human analyst[16]. In this vetting process an analyst must go through and validate the candidate TM. During this vetting process the analyst must validate given links along with adding links the automated process did not produce. This entire process must be fast, accurate, and certifiable. The former cannot be achieved without automated methods, however the latter requires a human to vet the TM.

This produces a process which we call assisted tracing or semi-automated tracing. Semi-automated tracing combines manual and fully automated tracing such that both the collection of links and link validation may be from either humans or software. When examining assisted tracing, the overall quality of the result is determined by the human-validated TM, not the TM produced by automated tool. Due to this, it is vital that the assisted tracing be done in such a way that the analyst consistently makes good decisions. If the automation produces a perfect quality TM, but the analyst can not recognize this while vetting, the process will fail. Figure 1.1 gives a visual representation contrasting manual, fully automated, and semi-automated tracing.

When evaluating the quality of various tracing methods, a common approach is to look at the accuracy of the final TM produced. To examine the accuracy of a TM, a common method is to compare the TM to the true or ideal TM (called the golden standard $)^{1}$. The accuracy measures used are precision, which is the percent of retrieved links that are true, recall, which is the percent of true links

\footnotetext{
${ }^{1}$ This "golden standard" is normally generated by the research team through manual tracing. We provide more information about the process we used to create this standard in Section 4.3.2
} 

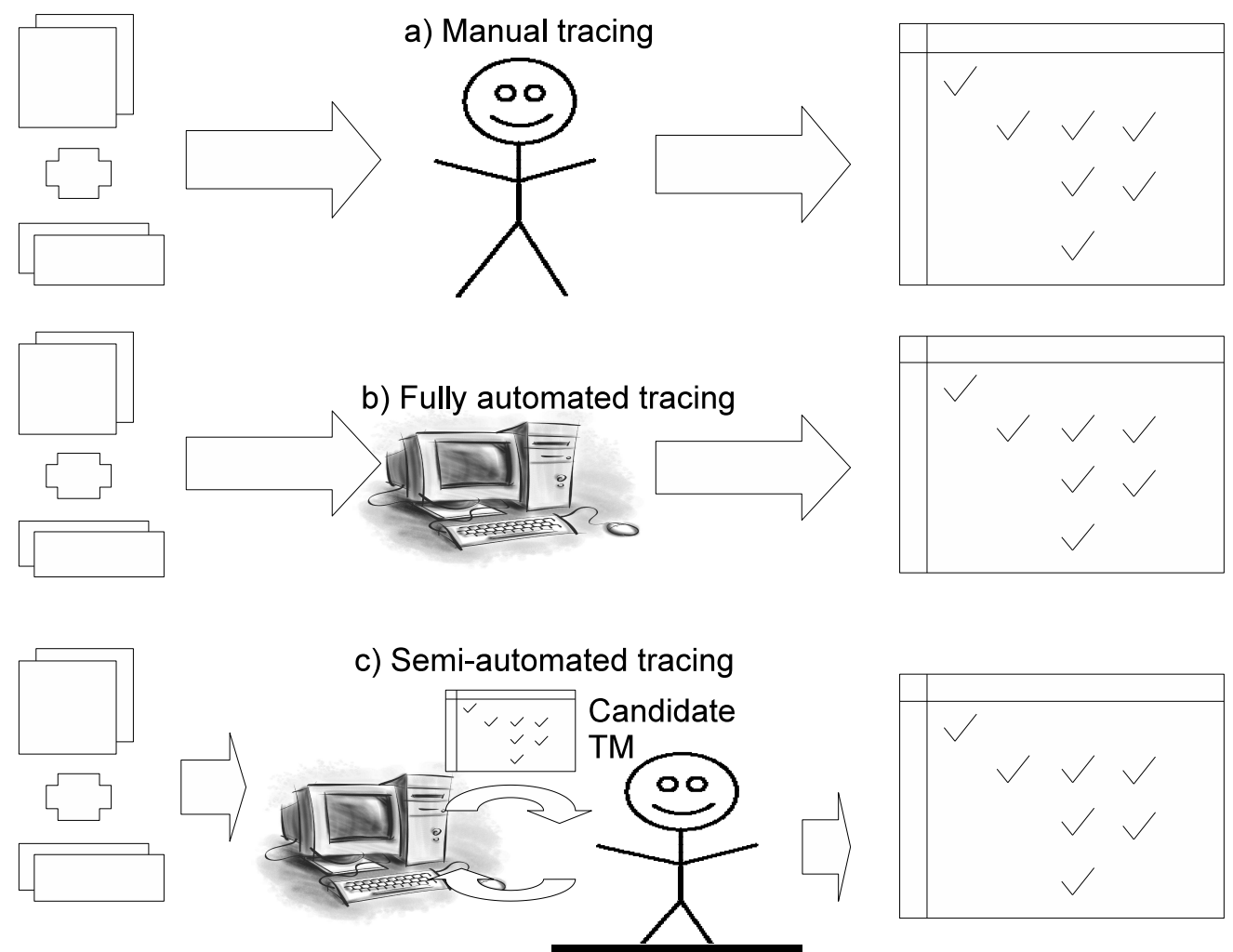

Figure 1.1: The overall process of a) manual tracing b) fully automated tracing c) semi-automated tracing.

that are retrieved, and $f$-measure, which is the harmonic mean between precision and recall. These measures are described more formally in section 2.3.

In 2005, Hayes and Dekhtyar began to examine the role of the analyst in the assisted tracing process. Specifically they asked whether higher-quality candidate TMs reliably lead to higher quality final TMs. The initial thoughts were:

1. The assisted tracing process would be "garbage-in - garbage-out": if an analyst was given a low accuracy candidate TM they would have a more difficult time than if given a high accuracy candidate TM.

2. Analysts would tend to improve the quality of the candidate TM. 
A small experiment was conducted to test these expectations and while the sample size was insufficient to draw solid conclusions, it did provide anecdotal evidence that neither assumption held.

The study of assisted tracing was next extended in 2009 - 2010 by Cuddeback et al. $[7,6]$. Their research gave additional support that the "good-in - goodout" scenario may not be the case. In fact, in $[7,6]$ analysts given some of the lowest accuracy candidate-TM improved them, while analysts vetting the highest accuracy candidate TMs decreased the overall accuracy.

The experiment described in $[7,6]$ was conducted using a BlueJ plug-in called ChangeStyle designed to format Java source code. This dataset contains 32 requirements, 17 test cases, and 24 true-validated links. This dataset is described in detail in section 4.3. The full dataset can be viewed in Appendix H.

Cuddeback et al.[6] collected and presented information about the change in accuracy between the initial TM and the final TM. The main way the analyst behavior was evaluated was by mapping the drift from initial TM to the final TMs accuracy as measured by recall and precision. This was illustrated by drawing vectors from the initial TM (represented by the tail) to the final TM (represented by the head of the vector) in precision-recall space.

In $[7,6]$ Cuddeback et al. noticed four different analyst behaviors based on starting precision and recall values. The behavior was based on the quadrant of the initial TM(see Figure 1.2): (1) low-precision, low-recall; (2) low-precision, high-recall; (3) high-precision, low-recall; and (4) high-precision, high-recall. Due to the importance of these observations to this thesis we provide an explanation of each of the four regions, how they are identified, and an overview of the behavior observed by participants in this region. Figure 1.2 summarizes the main results 


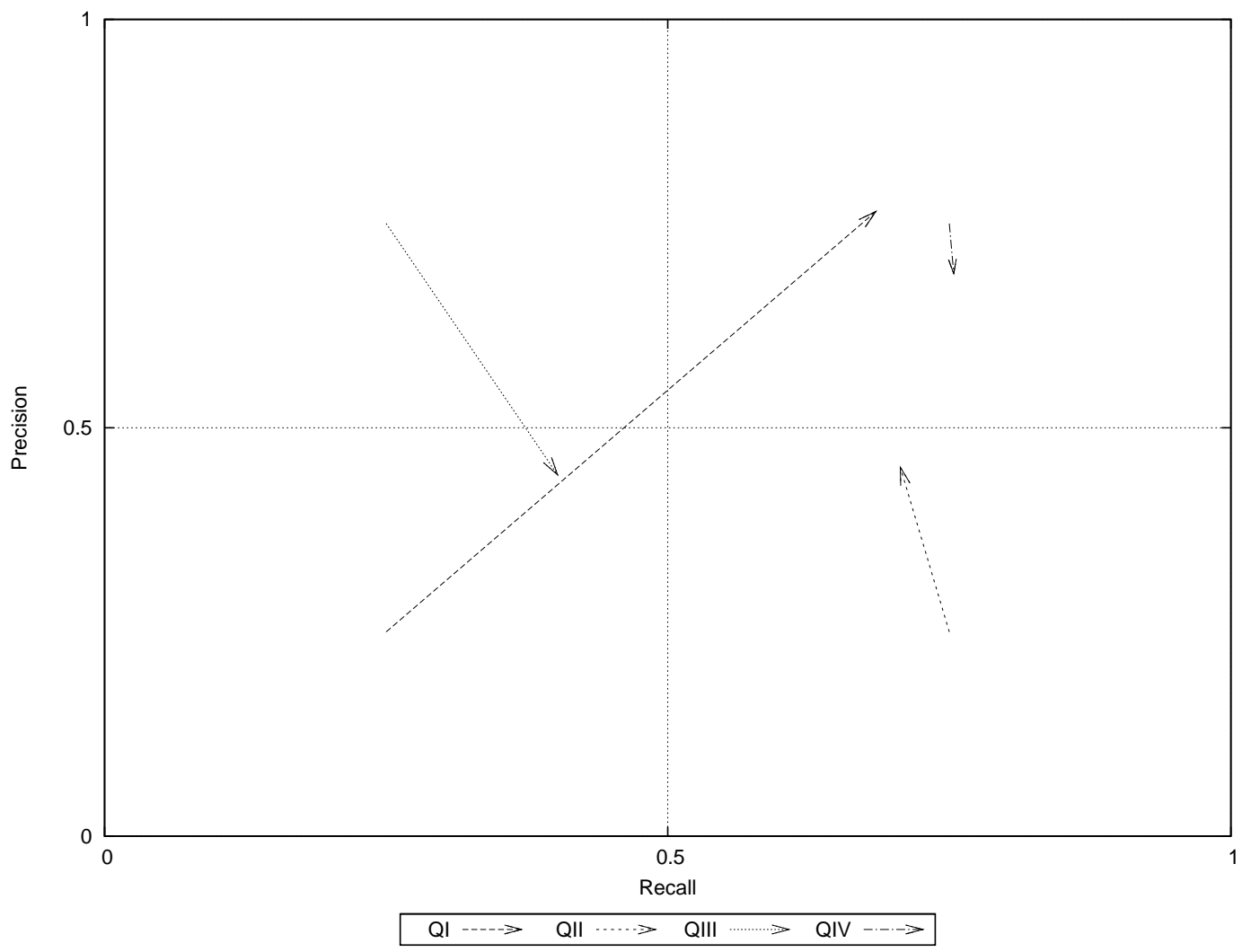

Figure 1.2: Average precision and recall drift from [6], separated in four quadrants

of $[6]$.

Low precision, Low recall. Initial TMs in this region, lower left corner of Figure 1.2, contained a very low percentage of the total correct links with many of the presented links being false positives i.e. incorrect links. The initial TMs in this region were 20-30 links in size. These where the lowest quality TMs presented to any analysts as measured by $f_{2}$-measure. The analysts performed quite well and were able to substantially improve the quality of their TM. They showed the ability to recognize valid links in their initial TM as well as retrieve valid links that were missing. While these analysts did not reach $100 \%$ accuracy, they showed the desirable trajectory and vetting behavior. 
Low precision, High recall. Initial TMs in this region, lower right corner of Figure 1.2, were the largest in the experiment with many valid links, but also with the false-positive links outnumbering the valid links. These TMs ranged from 34 to 110 links in size.

The analysts working with these TMs successfully identified and removed many of the false positives, however in the process, also removed some correct links. These analysts displayed a "weeding out" behavior where they rarely went looking for missing links but focused on candidate link vetting, often with correctness being a casualty. This is shown by the vector which increased in precision, however at the cost of recall. The final TMs submitted by analysts contained fewer false positives but did not completely remove them.

High precision, Low recall. Initial TMs in this region, upper left corner of Figure 1.2, were very small in size. The majority of links that were displayed were correct, however most of the correct links were still missing. These TMs were 6-14 links in size.

The analysts presented such TMs tended to understand that their candidate TMs did not contain enough links and went searching for additional links to add. When adding links the analysts did correctly recognize and add several valid links, however they also added many false positives. This is shown by the vector which increased in recall, however at the cost of precision.

High precision, High recall. The TMs in this region, upper right corner of Figure 1.2, were the highest quality matrices presented to the analysts. These TMs contained between 14 and 31 links, with the majority of the links being valid. 
The analysts given such TMs showed interesting behavior. Despite being presented with the highest quality TMs, they frequently produced TMs of decreased accuracy. In fact, out of the 11 participants with TMs in this region only one analyst increased the accuracy of their TM significantly, with three more maintaining their accuracy. The remaining seven participants decreased their TM accuracy by an average of almost $9 \%$ when comparing the $f$-measure. In this region analyst behavior was diverse; some analysts improved precision but decreased recall while others improved recall at the expense of decreasing precision.

The observations made by Cuddeback et al.[6] revealed interesting analyst behaviors. Considering these results, with semi-automated tracing, we can no longer rely solely on the accuracy of the automated methods as the criteria for the quality of the tracing process. In contrast, we must now acknowledge that the human analyst is a vital component in the traceability process and therefore design semi-automated systems which can account for and mitigate fallible behavior of humans.

The research in this thesis looks to continue where the study of Cuddeback et al. left off. First, we undertook a larger study to expand upon Cuddeback[6] and validate the results found in his experiment. We did this by running similar experiments with two additional tracing processes: one using a second traceability tool and the other asking participants to vet a candidate TM manually. Additionally we looked to gather more detailed information concerning what an analyst did while tracing. To accomplish this we integrated logging into our traceability tool which recorded user actions while vetting. We expanded Cuddeback's study of 33 analysts by adding 38 manual process participants and 13 participants who used a different software tool. 
By combining these new participants with the participants from Cuddeback, we were able to form a large enough sample to perform formal statistical analysis. Our experiments confirmed the findings of Cuddeback et al., both informally and formally with statistics.

After validating the results of Cuddeback et al. we expanded upon these findings. In the experiments Cuddeback et al. performed, the candidate TMs were constructed based on specified $\langle$ precision, recall $\rangle$ numbers using an ad-hoc generation process. We asked the question: how analysts interact with naturally generated TMs, so called as they are generated using real information retrieval and filtering techniques. We looked to answer this question by converting our software tool and experimental design to utilize real automated information retrieval(IR) methods and filters to produce naturally generated TMs. In this manner we changed the independent variables from initial precision and recall, used by Cuddeback, to the IR and filter methods that were applied to generate the candidate TM.

Additionally we modified the traceability tool to allow three different IR methods, six different filtering methods, and user feedback processing for a total of 36 potential combinations. We ran a pilot experiment using these variations to determine what the ideal IR and filter combination allowed analysts to consistently improve the quality of the candidate TM presented to them.

The contributions of this thesis are:

- Extended the Cuddeback et al. study by running their experiment using two additional set-ups.

- Conducted statistical analysis of the results of the experiments. 
- Built a new, extensible experimental tool and framework.

- Designed an experiment to examine the use of IR methods for semi-automated traceability tools.

- Conducted a pilot experiment using this new experimental setup.

The rest of this thesis is organized as follows: Chapter 2 covers background information on automated tracing, assisted tracing, and related research. Chapter 3 describes an overview and motivation behind all our experiments. Chapter 4 describes our experimental setup and tool design from our extended Cuddeback et al. study. Chapter 5 describes our results and analysis from these experiments. Chapter 6 describes our new experimental setup and tool design using naturally generated TMs. Chapter 7 shows the results and analysis from this experiment Finally Chapter 8 gives comments on future work and Chapter 9 concludes. 


\section{Chapter 2}

\section{Background and Related Work}

Gotel and Finkelstein define requirements traceability as "the ability to describe and follow the life of a requirement, in both a forwards and backwards direction (i.e., from its origins, through its development and specification, to its subsequent deployment and use, and through all periods of on-going refinement and iteration in any of these phases)" [14].

Requirements tracing is defined as the process of establishing requirements traceability. In practice this involves linking high and low-level elements from different artifacts. An artifact can be any by-product of a software life cycle. Some examples of artifacts can be a requirements document, design document, or code. An element is a distinct part of an artifact that can be traced. Examples of elements are a requirement from a requirements document; a test case from an acceptance test document; a class in source code or design documents; and a section of user documentation.

When analysts preform requirements tracing they normally produce a requirements traceability matrix (RTM) often called a traceability matrix (TM). A 
TM visually shows the relationship between high and low elements in different artifacts.

A connection between high and low elements in a TM is called a link. A candidate link is any possible link between two artifacts. Thus, for two artifacts with 10 and 5 elements respectively, there are $10 \times 5=50$ candidate links. To measure the accuracy of a TM, there must be a notion of correctness for the TM links. A link that is correct is referred to as a true link and a link that is incorrect as a false link.

Hayes et al. [16] break the tracing process into a seven steps as outlined in Figure 2.1. Steps 3-6 are the research focus of automated traceability.

\subsection{Automated Requirements Tracing}

Traditionally, tracing is done manually. An analyst examines each candidate link and renders a decision on wheter it is a valid link.

In practice, manual requirements tracing can be a slow, laborious and error-

prone task. To alleviate these issues research has looked at ways that can speed up the process for the analyst. Much of the existing research utilizes information retrieval techniques to automate the process.

When requirements tracing is preformed utilizing the assistance of automated methods, a common process is to provide the analysts with a TM for the analyst to validate. The TMs provided to the analysts are candidate TMs. Once an analyst validates the TM the TM then becomes a final TM, or the TM that the analyst validates as correct.

The problem of tracing textual requirements can be viewed as an information 


\begin{tabular}{llcc}
\hline Technique & Dataset & Recall & Precision \\
\hline \hline TF-IDF [2] & LEDA, Albergate & $86-100 \%$ & $6-18 \%$ \\
\hline Probabilistic Method [2] & LEDA, Albergate & $94-100 \%$ & $6-19 \%$ \\
\hline Latent Semantic Indexing [25] & LEDA, Albergate & $91-100 \%$ & $16-25 \%$ \\
\hline Probabilistic Method [5] & IBS & $87 \%$ & $51 \%$ \\
\hline TF-IDF [15] & MODIS [27, 23] & $63 \%$ & $39 \%$ \\
\hline TF-IDF with Thesaurus [15] & MODIS [27, 23] & $85 \%$ & $40 \%$ \\
\hline TF-IDF with feedback [16] & MODIS [27, 23], CM-1 [26] & $90 \%$ & $80 \%$ \\
\hline Probabilistic tracing model [4] & HIPAA [4] & $52-100 \%$ & $2-14 \%$ \\
\hline Machine learning tracing [4] & HIPAA [4] & $57-100 \%$ & $6-58 \%$ \\
\hline
\end{tabular}

Table 2.1: Summary of research in IR techniques for automated traceability [6].

retrieval problem. Information retrieval is focused on solving the problem of finding documents from a collection that are relevant to a textual query [15]. In requirements tracing, the high-level elements can act as queries and the low-level elements are the collection that the query is ran on [17]. Several research groups have shown that information retrieval techniques are successful at generating candidate links. These research groups include Antoniol et al.[2], Marcus and Maletic[25], Huang et al.[5], Hayes and Dekhtyar[16], Zou et al.[32], and Oliveto et al.[28] Table 2.1 summarizes the current research in methods for candidate link generation when comparing based on their recall and precision measures. These research methods vary between vector models, machine learning models, among others.

For a more detailed look at information retrieval techniques see section 2.2.

\subsection{Information Retrieval Techniques}

Information retrieval is the study of mapping from one set of text documents to a second set of documents. In general terms, information retrieval studies the 
1. Identify the elements of both artifacts to be traced.

2. Assign unique identifiers for each traceable element.

3. For each high-level element, locate all matching low-level elements.

4. For each element in the child document, locate a parent element.

5. Determine if each high-level element has been satisfied.

6. Prepare a traceability matrix.

7. Prepare a summary of the traceability between the two documents.

Figure 2.1: The tracing process[16].

problem of finding relevant documents in a collection given specified queries[15]. Information retrieval attempts to determine how relevant a document is when compared to these queries. When one applies general information retrieval methods to a specific requirements tracing task, one set of documents is used as the collection to search and the second set is used as the list of queries. In this manner the IR methods create a relevance mapping from the two sets of documents (such as requirements to test cases)[17].

One of the most popular ways to create this relevance mapping in information retrieval is using keyword-based methods. In these methods, documents are represented by a collection of keywords they contain. When computing the relevance score of documents, several techniques including Vector-Space model(VSM)[30], latent semantic indexing(LSI) $[21,9]$, and probabilistic IR $[12,2]$ are used for tracing. Of these options, one of the most commonly used techniques is vector-space model. This is the model we use for our research. 


\subsubsection{Vector Space Model}

A vector-space model is a model that represents text documents as vectors of term weights such that each element of the vector represents a term and the weight of that term is the term's relative importance in that document.

The most simple way to weigh terms is to use term frequency. Several other methods have been proposed and are commonly used to represent the terms in a document. Common methods include Term Frequency-Inverse Document Frequency(TF-IDF) and Okapi.

\section{TF-IDF}

TD-IDF stands for "term frequency - Inverse document frequency". In TFIDF, each term is given a weight computed as the term frequency (the number of occurrences of the term in the document), possibly normalized, multiplied by the inverse document frequency of this term. Inverse document frequency is the size of the document collection divided by the number of documents a given term is in. Using this formula the more frequent a term is in a document, the more important that term becomes. However, the more frequently a term is found in a given collection, the less important it becomes for telling documents apart.

This is shown mathematically as $w_{i}=t f_{i}(d) * i d f_{i}$. Here $t f_{i}(d)$ is the term frequency and $i d f i$ is the inverse document frequency. These terms are computed as $t f_{i}(d)=f_{i j} / \max \left(f_{1 j}, f_{2 j}, \ldots, f_{M j}\right)$ and $i d f_{i}=\log _{2}\left(n / d f_{i}\right)$. In these equations $t f_{i}(d)$ is the normalized term frequency, $d f_{i}$ is the total number of documents containing the $i$ th term in the collection, and $n$ is the total number of documents in the collection. This method weighs the terms that are more specific to a document higher than terms that are more common in the entire document 
collection[29]. In tracing applications, document and query weights are normally computed the same way using the TF-IDF weighting scheme shown above.

TF-IDF uses cosine similarity to compute relevance between a document and a query (2nd document). This similarity is formally defined mathematically as

$$
\operatorname{sim}\left(d_{j}, q\right)=\cos \left(d_{j}, q\right)=\frac{\left(d_{j} \cdot q\right)}{\left\|d_{j}\right\| \cdot\|q\|}=\frac{\sum_{i=1}^{M} w_{i j} \cdot w_{i q}}{\sqrt{\sum_{i=1}^{M} w_{i j}^{2} \cdot \sum_{i=1}^{M} w_{i q}^{2}}}
$$

\section{Okapi}

Another common information retrieval technique used is Okapi. Okapi looks to compensate for the disparity in the size of the query and the size of the document being queried. It does this by modifying the term weights to account for the length of the document searched and the average length of the document in the collection[31].

Okapi can be viewed as either modifying term weights or modifying the similarity computation. In practice the latter view is more convenient as it uses the TF-IDF term weights, and thus, does not require special processing. Okapi replaces the simple cosine similarity between document and query with the formula shown below.

$$
\operatorname{okapi}\left(d_{j}, q\right)=\sum_{t \in d_{j, q}} \ln \frac{n-d f_{i}+.5}{d f_{i}+.5} \times \frac{\left(k_{1}+1\right) \cdot f_{i j}}{k_{1} \cdot\left(1-b+b \cdot \frac{d l j}{a v d l}\right)+f_{i j}} \times \frac{\left(k_{2}+1\right) \cdot f_{i q}}{k_{2}+f_{i q}}
$$

In this formula the first part is computing the inverse document frequency. The part takes the number of documents that the term is not in $\left(n-d f_{i}\right)$ and divides by the number of documents the term is in $\left(d f_{i}\right)$. In practice, both the numerator and denominator are increased by 0.5 to ensure that the term does not go to 0 (e.g. if the specified term is in all documents so $n-d f_{i}$ would be 0 ).

The second part of the computation is calculating the term frequency in the 
document, adjusted by the document length. The parameters are defined as: $k_{1}$ is a normalization parameter (normally between 1.0 and 2.0) to normalize term frequency for document $d_{j} . f_{i j}$ is the frequency of the term, $b$ is the normalization parameter for document length to account for the trend that documents tend to be longer than queries, this value is usually 0.75 . Finally $d l_{j}$ is the length of the document and $a v d l$ is the average length of a document in the collection. The document length comes into play: when the value of $k_{1}$ is larger, the difference of weights becomes a larger factor in the similarity calculation.

Finally, the third part of the computation is calculating the term frequency in the query with out adjusting for the document length. The parameter $k_{2}$ is a normalization parameter to normalize for query $q$. This parameter is normally between 1 and 1000, a larger value weights the occurrence of a term in query $q$ with a higher weight.

In our implementation we utilize the following values for each of the parameters:

- $k_{1}=1$

- $b=0.5$

- $k_{2}=1$

\section{$2.3 \quad$ Measures}

In order to determine the quality of a TM we define several mathematical metrics. The standard metrics used are precision and recall, and $f$-measure, which is the harmonic mean of precision and recall. Additional metrics, selectivity 
and lag, are used to assist in objectively measuring the effort required by a human analyst when they vet a TM.

To assist with the mathematical definitions we introduce a few variables. Let $\mathcal{H}$ be the set of high-level elements of size $M$ and let $\mathcal{D}$ be a set of low-level elements of size $N$. For a particular element $q \in \mathcal{H}$, let $n_{q}$ be the number of candidate links between $q$ and the low-level elements in $\mathcal{D}$ that exist in the TM. Let $r_{q}$ be the number of those links which are correct and $R_{q}$ be the actual number of correct links between $q$ and the elements in $\mathcal{D}[16]$.

Recall. Recall is the percentage of correct links that were retrieved [16]. Given a requirement $q$, the recall for the individual requirement is $\frac{r_{q}}{R_{q}}$. The overall recall for the entire document is defined formally in (2.1). Intuitively, recall is the percentage of correct links that were discovered during the tracing process. It is the sum of the correct links found for the TM divided by the sum of the actual number of correct links for the true TM [16].

$$
\text { recall }=\frac{\sum_{q \in \mathcal{H}} r_{q}}{\sum_{q \in \mathcal{H}} R_{q}}
$$

Precision. Precision is the percentage of retrieved candidate links that are correct [16]. Given a requirement $q$, the precision for the individual requirement is $\frac{r_{q}}{n_{q}}$. The overall precision for the entire TM is defined formally in (2.2). Overall precision is the sum of the correct links found divided by the total number of links found for the TM [16]. 


$$
\text { precision }=\frac{\sum_{q \in \mathcal{H}} r_{q}}{\sum_{q \in \mathcal{H}} n_{q}}
$$

$f$-measure. $f$-measure is the harmonic mean of recall and precision. Defined formally in (2.3), it represents a balance between recall and precision and can be weighted to emphasize one metric or the other. $b=1$ weights recall and precision equally, $b<1$ favors precision, and $b>1$ favors recall.

$$
f_{b}=\frac{1+b^{2}}{\frac{b^{2}}{\text { recall }}+\frac{1}{\text { precision }}}
$$

In this thesis, following [16], we use the $f_{2}$-measure, i.e., the $f$-measure for $b=2[16] . f_{2}$ is used because better recall means fewer errors of omission while better precision means fewer errors of commission, and [16] observed that fixing errors of omission is harder than fixing errors of commission.

Selectivity. When an analyst performs manual requirements tracing the analyst essentially has to consider all the candidate links. In order for an analyst to trace an artifact with $M$ elements to a second artifact with $N$ elements, the analyst would have to consider $M \times N$ candidate links. Selectivity is the fraction of these links that are returned by the automated method during the tracing process. Unlike other measures where bigger is better, a lower selectivity value is preferable as the analyst has fewer candidate links to validate.

Selectivity is the number of the candidate links returned divided by the total number of candidate links [16]. This is defined formally in (2.4). 


$$
\text { selectivity }=\frac{\sum_{q \in \mathcal{H}} n_{q}}{M \cdot N}
$$

Precision, recall, and selectivity need to be considered in combination. As an example, it is easy to achieve $100 \%$ recall if we do not regard precision; we can return a TM with all candidate links. Similarly, to reach $100 \%$ precision we could return a candidate TM with only one valid link. Both of these examples are not ideal and show the necessity of considering these measures in combination.

Lag. Lag is a measure of the separation between true and false links. For a requirement $q,(q, d)$ represents a true link in the candidate links. $\operatorname{lag}(q, d)$ is the number of false links that have higher relevance scores than $(q, d)$. The overall lag for an artifact is the average of the lags for all true links in the list of candidate links. This can be formally defined in Equation (2.5).

Let $\mathcal{T}$ be the set of true links in the candidate links.

$$
\operatorname{lag}=\frac{\sum_{(q, d) \in \mathcal{T}} \operatorname{lag}(q, d)}{|\mathcal{T}|}
$$

Table 2.2 display the acceptable recall, precision, and lag as defined by Hayes et al. in [16]. These levels have not been empirically determined however are the best acceptable measures currently available and are based on industry experience. 


\begin{tabular}{lccc}
\hline Metric & Acceptable & Good & Excellent \\
\hline \hline Recall & $60-69 \%$ & $70-79 \%$ & $80-100 \%$ \\
\hline Precision & $20-29 \%$ & $30-49 \%$ & $50-100 \%$ \\
\hline Lag & $3-4$ & $2-3$ & $0-2$ \\
\hline
\end{tabular}

Table 2.2: Acceptable levels for recall, precision, and lag [16].

\subsection{The Human Side of Automated Traceability}

In 2005 Hayes and Dekhtyar asked whether it is, in fact, true that more accurate initial candidate TMs will lead to more accurate final TMs [19, 18]. This was, to our knowledge, the first study of the analyst's role in the TM validation process. While their study only contained four analysts, it provided some anecdotal evidence that this may not be the case.

In the experiment, four analysts were given candidate TMs of varying accuracy and asked to validate them. The results of this experiment are shown in Figure 2.2. In this figure, each vector represents the change in accuracy from the initial to the final TM. The tail of each vector represents the initial precision and recall. The head of the vector represents the precision and recall of the final analyst-validated TM. The key observations from this study are that regardless of starting TM accuracy, analysts make errors of omission and commission. Another interesting observation is that analysts could not agree what the "true" TM was [19].

This study was expanded upon in $[7,6]$ which conducted a larger study to test the theory. In this study analysts where asked to validate a dataset ChangeStyle, a Java code formatter plugin for the IDE BlueJ. The full dataset is shown in Appendix H.

This dataset was chosen because: (a) the domain was easily understood by 


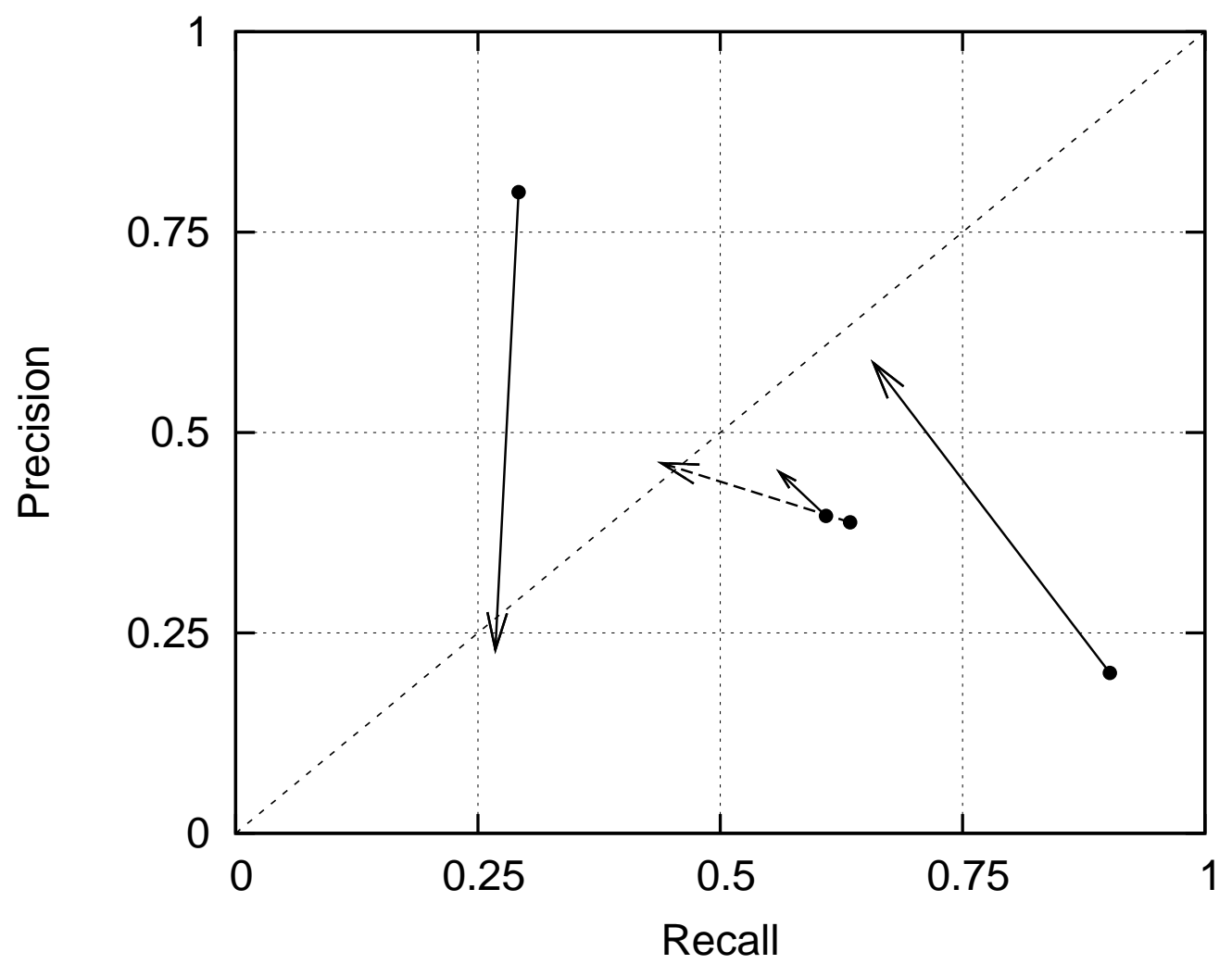

Figure 2.2: Results from a pilot study [19]. Arrows indicate change in accuracy after analyst corrections.

study participants, and (b) its size makes tracing validation tasks achievable in about one hour. The dataset contained 32 high- and 17 low-level elements. The golden standard TM was validated by the research team and contains 24 links ${ }^{1}$ $[6]$.

Similar to the study conducted in [19], participants were assigned an initial TM of varying precision and recall. These analysts were then asked to validate the candidate TM using a software tool and produce a final TM. The participant performance was than mapped using vectors were the tail of the vector represents

\footnotetext{
${ }^{1}$ Cuddeback et al. conducted their experiments and analysis using a golden standard with only 23 links. Upon further analysis a missing link was found, in this thesis all data is reported using the true 24 link golden standard. While specific numbers changed slightly, the overall conclusions of $[7,6]$ are not affected by this change.
} 


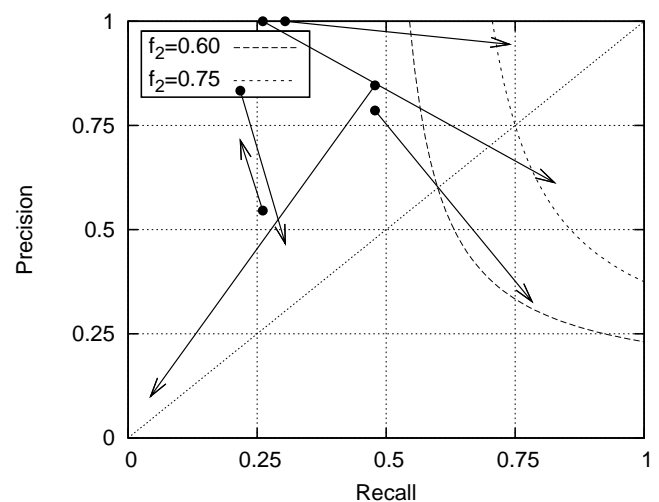

(a)

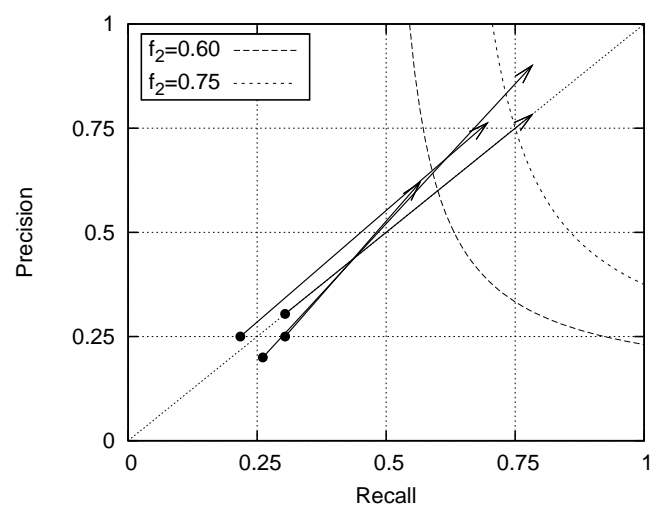

(c)

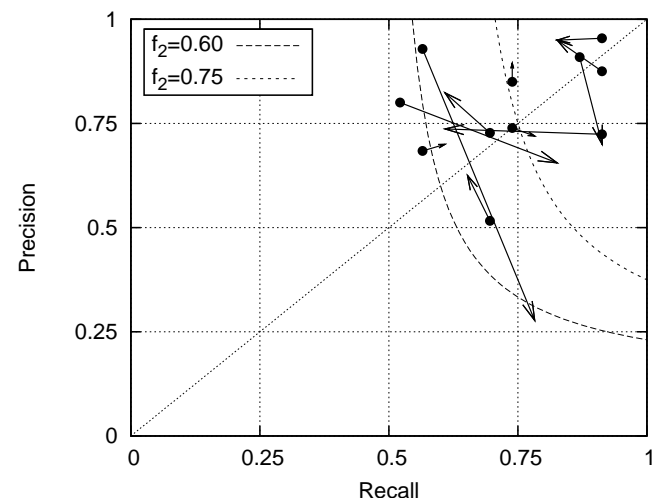

(b)

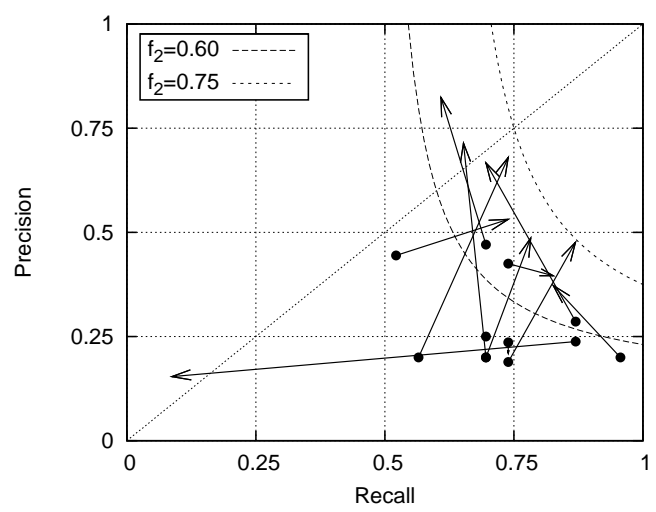

(d)

Figure 2.3: Change in precision and recall for participants from the Cuddeback et al. study in the four regions of the precision-recall space[6]. 
the initial precision-recall and the head represents the final TM precision-recall. To improve visibility, analyst performance was divided into four regions shown in Figure 1.2. The full results for the experiments, separated by the quadrant in which the accuracy of the initial candidate TM fell, is displayed in Figure 2.3.

Cuddeback et al. [7, 6] did not provide statistical analysis of the results. However, he made a number of important observations about the possible trends and their causes $[6]$.

1. Participants were unable to recover the true TM or even reach a consensus of what that TM should be.

2. Participants given the highest quality candidate TMs almost uniformly degraded their TMs accuracy while performing validation.

3. Participants who received candidate TMs with low precision and recall showed a pattern of some of the largest improvements in quality.

Cuddeback et al. also observed that analyst behavior was tied to the analysts initial candidate TM accuracy. Specifically they noticed four distinct regions in the precision-recall with different analyst behavior. These regions were low-precision, low-recall; low-precision, high-recall; high-precision, low-recall; and high-precision, high-recall. Each of these regional behaviors is described bellow and described in more detail when comparing with our results in Section 5.4.2. These regions are shown in Figure 2.3.

Low-precision, Low-recall. Analysts with initial TMs from this region dramatically improved both precision and recall. These participants demonstrated some of the highest improvements in accuracy as observed by $f_{2}$-measure. 
Low-precision, High-recall. Analysts with initial TMs from this region improved precision, however this was often at the cost of decreasing the recall. Cuddeback et al. conjectured that analysts with initial TMs from this region concentrated on determining errors of commission (false positives) without concentrating on errors of omission. This led to removing many of the false positives, however errors of judgement also led to removing correct links.

High-precision, Low-recall. Analysts with initial TMs from this region tended to improve recall while decreasing precision. Cuddeback et al. conjectured that participants concentrated on solving errors of omission, however during this process additional false positives were also added due to errors in judgement.

High-precision, High-recall. Analyst behavior in this region was diverse; some improved precision but at the cost of decreasing recall while others did the opposite. The one unifying factor was that nearly every analyst decreased the overall TM accuracy. In fact, despite the analysts being presented with the highest accuracy candidate TMs, only one analyst increased the accuracy of their candidate TM.

In 2009 Egyed et al. conducted a study that examined the effect of analyst effort on the number and quality of recovered links when tracing a larger software system[13]. In this study Egyed et al. asked 100 analysts to perform trace link recovery using a software tracing tool. The participants were asked to record a link/no-link decision on potential links from requirements to $\mathrm{C}++$ classes or methods. There were two significant differences in their study when compared to the Cuddeback study:

1. The software tool was designed for data collection, it did not attempt to 
automate any portion of the tracing process.

2. The analysts were asked to trace from scratch rather than being provided an initial candidate TM.

In addition, Egyed et al. mainly focused on the analyst's effort required to make decision as compared to Cuddeback who focused on the final accuracy of the TM. While the primary focus of Egyed's study was the analyst effort, this study also supported the findings of Cuddeback et al. that humans are fallible in their work with candidate TMs.

In particular, Egyed looked at link verification and what percentage of links were accepted and rejected. His findings were that analysts are good at correctly identifying what he called no-link votes, or incorrect links with a $95 \%$ correct decision rate overall. However, when examining link votes, or adding a link into the final TM, analyst performance is significantly lower, only $50 \%$ correct decision rate. He also saw that as analyst experience increased (from less than 2 years to more than 4 years experience) the analyst would make fewer decisions, however their overall decision accuracy would increase. However, Egyed did state this increase in performance was only weakly correlated to analyst experience[13].

Finally, Egyed et al. also explored trace effort and quality of different phases. From their data they suggest trace validation should be done incrementally in short sessions. In particular they saw the task of validation has a short learning phase $(<20$ minutes), reaches optimum $(<60$ minutes $)$, but leads to fatigue shortly after ( $<90$ minutes). They found that the additional effort does not imply better trace quality. In fact they found two categories of validation: fast + accurate and slow + inaccurate[13]. This is similar to the findings of Cuddeback et al. which showed there was not a correlation between analyst effort and the 
final link quality[6].

\subsection{Traceability tools}

Several software tools have been created by industry and research groups to attempt to assist with the process of creating a TM. Poirot is one such tool which looks to generate traces between requirements, design elements, code, and other artifacts[24]. Poirot is a web-based tool which uses information retrieval techniques to preform either artifact-to-artifact queries or free-text queries. Poirot was designed to be a enterprise tool focusing on obtaining high-recall. This tool focuses on believability, specifically accuracy and scalability. They do not focus on discernibility or endurability.

ADAMS Re-Trace is another tool to aid in the traceability process[8]. This tool was implemented as an Eclipse plug-in which allowed developers access to traceability information. This is targeted to assist with change-analysis and change-management during the development process[8]. The main focus of this tool was to provide immediate access to traceability data to the developer and not necessary examine how individuals would use the tool or what makes the tool easy to use (utility and discernibility).

RETRO is a research tool developed by Hayes et al. for the purpose of assisting in automated trace recovery [20]. This tool was originally designed as a framework to allow researchers to test their IR methods. The researcher's view of RETRO evolved and became focused on creating a special-purpose requirements tracing tool to allow for simple independent verification and validation (IV\&V)

tasks[20]. While the initial tool was designed to support IV\&V, the tool was further modified to support human-centric research. Cuddeback et al. modified 
RETRO to allow delivery of pre-generated TMs to human analysts[6]. Additionally RETRO has also evolved into a more user-friendly version, RETRO.net[11]. More detailed information on RETRO and RETRO.net is provided in section 4.1 .

TraceLab is another tool designed to be an extensible framework for traceability research[3]. This tool was developed by the Center of Excellence for Software Traceability (CoEST). TraceLab was designed with the knowledge that it can take new researchers a long period of time to develop a research environment for traceability-centric research. In addition, it can be painful for researchers to perform rigorous evaluations of their current research. TraceLab is designed with the following target users: researchers executing experiments, $\mathrm{PhD}$ students developing new experiments, and industry adaptors who will use TraceLab for trace tasks such as IV\&V.

To make this tool accessible to all these groups TraceLab was designed as a visual precedence graph such that one task in the experiment must be completed prior to the next one beginning. In addition, TraceLab is designed with the option to share research components. This allows cross research group collaboration as well as quick plug-in-play startup. Finally TraceLab is designed with a player mode such that experiment participants (or industry) can easily use the tool without requiring full developer features[3]. 


\section{Chapter 3}

\section{Overview of Experiments}

Our study looks to continue where the study of Cuddeback et al. left off[6]. Like Cuddeback, we concentrated on what analysts do when vetting candidate TMs. Our goal is to determine how human analysts vet the candidate TMs and use this knowledge to improve the semi-automate tracing process. Specifically, we want to study the following questions:

- How do human analysts transform the requirements traceability information from candidate TMs into final TMs?

- What are the main factors that influence the analyst's vetting performance?

To meet these goals we looked to expand upon Cuddeback et al. and solve some of the limitations presented in this study[6]. Specifically the limitations we looked to address are:

1. Cuddeback et al. had only 33 data points in their study[6].

2. Cuddeback et al. experimented and collected data on only one tracing process $[6]$. 
3. Cuddeback et al. did not present any formal data analysis, only informal observations $[6]$.

4. Cuddeback et al. utilized only one dataset for their experiments [6].

5. Cuddeback et al. utilized ad-hoc generation of candidate TMs[6].

To address these limitations we conducted two sets of experiments. Our initial experiment addressed limitations 1-3. Specifically we expanded the experimental framework presented by Cuddeback et al.[6] by adding two additional tracing procedures; manual tracing as well as utilizing a second tracing tool. By adding these additional processes we were able to partially address the internal validity threat presented in [6] and the limitation \#2 presented above.

Using these additional tracing procedures we were able to conduct experiments in three courses at two universities. Through these experiments we were able to increase our total sample size by over 2.5 times, from 33 presented in Cuddeback et al. to a total of 84 participants. This larger data sample allowed us to address limitations \#1 and \#3 from above.

With this larger data set we are able to observe trends on a larger scale. In addition to the informal observations, presented in Cuddeback et al.[6] and confirmed in our work, we are able to also examine the results more formally. In order to answer question \#2 from our goals we utilized statistical analysis to observe the impacts each independent variable had on our dependent variables; final precision, recall, and $f_{2}$-measure. This formal analysis directly addressed limitation \#3 from above.

While our first experiment confirmed many of the findings of Cuddeback et al. both informally and formally, we looked to examine more and see how human 
analysts would interact with naturally generated TMs. To accomplish this we created a new experimental framework to address limitations \#4 and \#5.

To address limitation \#4, we moved beyond using a single dataset and introduced a second dataset into our experiments. This second dataset allowed us to partially address the external validity threat presented by Cuddeback et al. where results can not be generalized. Using this second dataset we can now examine more generally how analysts interact when vetting candidate TMs as compared to examining how they vet only for a single dataset.

In addition our second experimental framework allowed us to directly address limitation \#5. Cuddeback et al. used ad-hoc or artificially generated TMs where they manually controlled how the TM was created and deployed to the user. In contrast our experimental framework employed what we are calling naturally generated TMs, or TMs generated by real IR methods and filters. In this way, where Cuddeback et al. directly controlled the precision and recall of the candidate TM, we now indirectly control these attributes.

We do this by employing real IR methods to produce a candidate TM. After candidate TM generation we can apply various filtering functions to this candidate TM to control how many links are recommended by the system to the analyst and in what manner. Additionally, our experimental framework allows us to control the user interface with which the analyst interacts with the system. By controlling this we are able to control what links the analyst sees, in what order, and in what manner. Finally, we also have the ability to control if the user decisions on candidate links in the past should have influence in what candidate links will be shown in the future. This process is called user feedback processing. 
This experimental framework allows for a wide variety of combinations to be tested on analysts to determine the ideal setup for a semi-automated tracing tool. What we describe in brief above and in detail in Chapter 6 is our full experimental framework. We conducted a pilot study utilizing this new framework and targeted a subset of the potential features, specifically IR method, filter used, and filter value.

What follows is expanding upon these experiments and our findings. In Section 4 we describe the experimental design and setup for our initial experiment extending the study of Cuddeback et al. Section 5 summarizes our results, both informal observations and the formal statistical analysis from all three studies. Section 6 describes our new experimental framework utilizing naturally generated TMs. Section 7 describes our results and analysis from analysts utilizing naturally generated TMs. 


\section{Chapter 4}

\section{Experimental Design using \\ Artificially Generated TMs}

Our experiments were conducted in multiple cohorts summarized in table 4.1. To give a broader overview of the full state of research table 4.1 includes the Cuddeback et. al study as cohort $1^{1}$. In addition, two additional cohorts have been added, cohort 2, an experiment where the participants were asked to trace manually and cohort 3, an experiment where the participants used a new traceability tool RETRO.net. A more detailed explanation of this tool vs. original RETRO is included in the next section 4.1.2. A summary of the research is shown in figure 4.1. In this figure we show how the Cuddeback et al. findings

\footnotetext{
${ }^{1}$ We include the results of Cuddeback et al. even though we did not conduct the experiments as we utilize their results when performing in-depth analysis.
}

\begin{tabular}{cllc}
\hline Cohort & Date & Tool used & Participants \\
\hline \hline 1 & May 09-Apr 10 & RETRO & 33 \\
\hline 2 & Nov 10 & Manual & 38 \\
\hline 3 & Dec 10 & RETRO.net & 13 \\
\hline
\end{tabular}

Table 4.1: Summary of experimental cohorts 
are combined with the results of additional studies we conducted to give us data which we can run statistical analysis on leading to statistically significant results. These results are presented in brief in section 5.5 and in detail in [10].

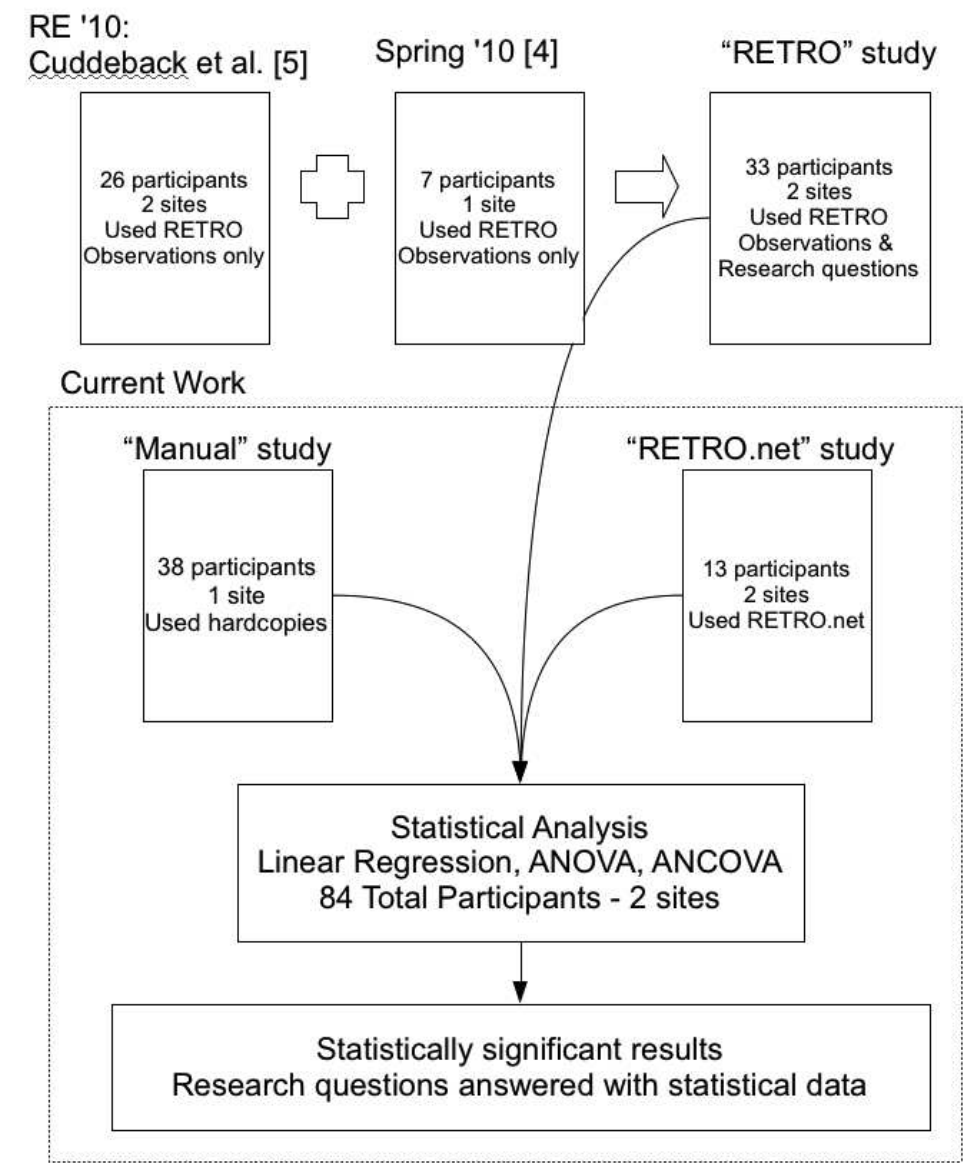

Figure 4.1: Overview of our research expanding upon the results of Cuddeback et al. 


\subsection{Experimental Tools}

Two of the three cohorts utilized software to deliver candidate TMs to the participants. The remaining cohort utilized hardcopy artifacts.

Two different tools were used. Cuddeback et al. used a traceability tool, REquirements TRacing On-target (RETRO)[20]. Our experiments utilized a streamlined version of this tool, RETRO.net[11].

\subsubsection{RETRO}

REquirements TRacing On-target (RETRO) is an automated requirements tracing tool which uses vector space IR methods to suggest candidate TMs to an analyst[20]. RETRO consists of three components: Information Retrieval toolkit, user feedback processing, and a GUI front end. The IR methods and feedback processing is written in $\mathrm{C}++$ and the GUI is written in Java. The components interact by writing and reading XML files to the hard drive. The RETRO UI accepts input from the user, modifies the XML files, and passes the files to the feedback processor.

RETRO's UI has two main displays, TRACE and BROWSE, accessible via tabs. The TRACE UI, shown in figure 4.2(a), displays the results from the IR methods allowing the analyst to confirm or reject candidate links. In contrast, the BROWSE UI, shown in figure 4.2(b), allows the user to view all potential links, not just the links obtained by IR methods. In this way the tool allows the analyst to correct for errors of omission.

In the Cuddeback et al. experiment[6] the original RETRO was modified for experiment use. A log-in prompt and a back end was added which replaced the IR 


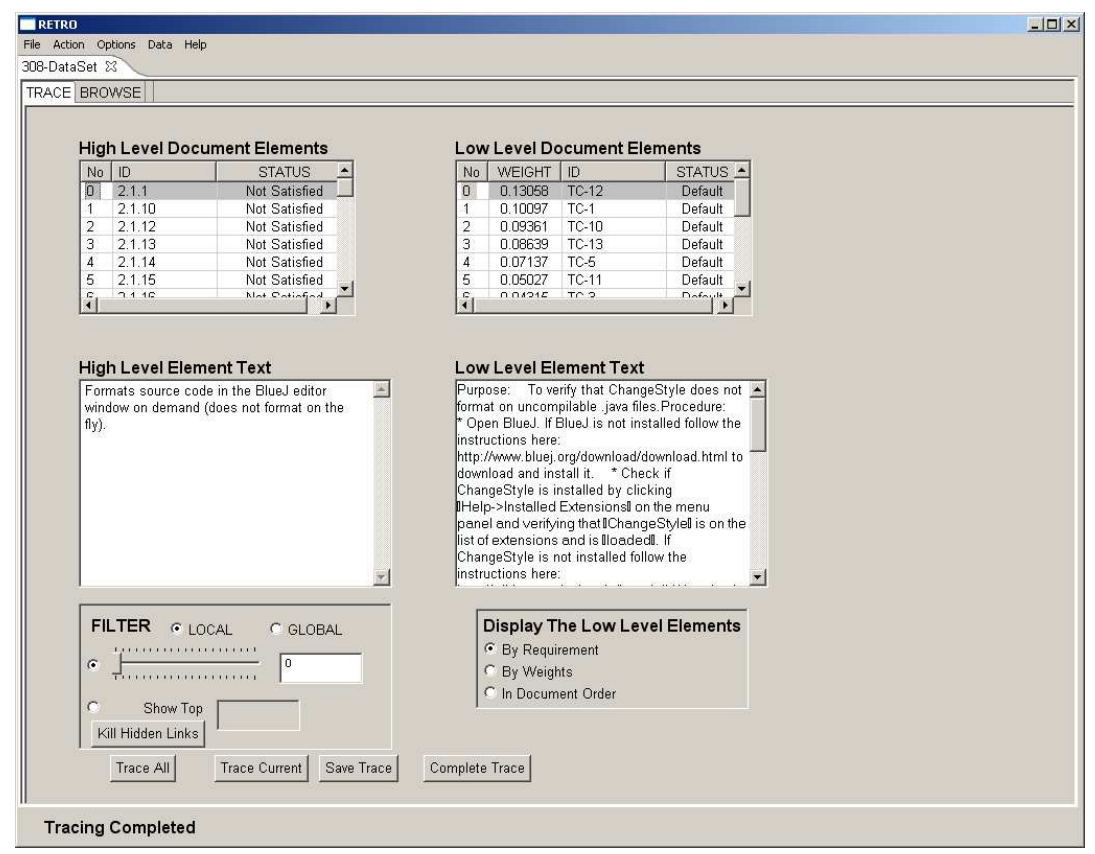

(a) RETRO's "trace" tab displays recommended links for the analysts to vet.

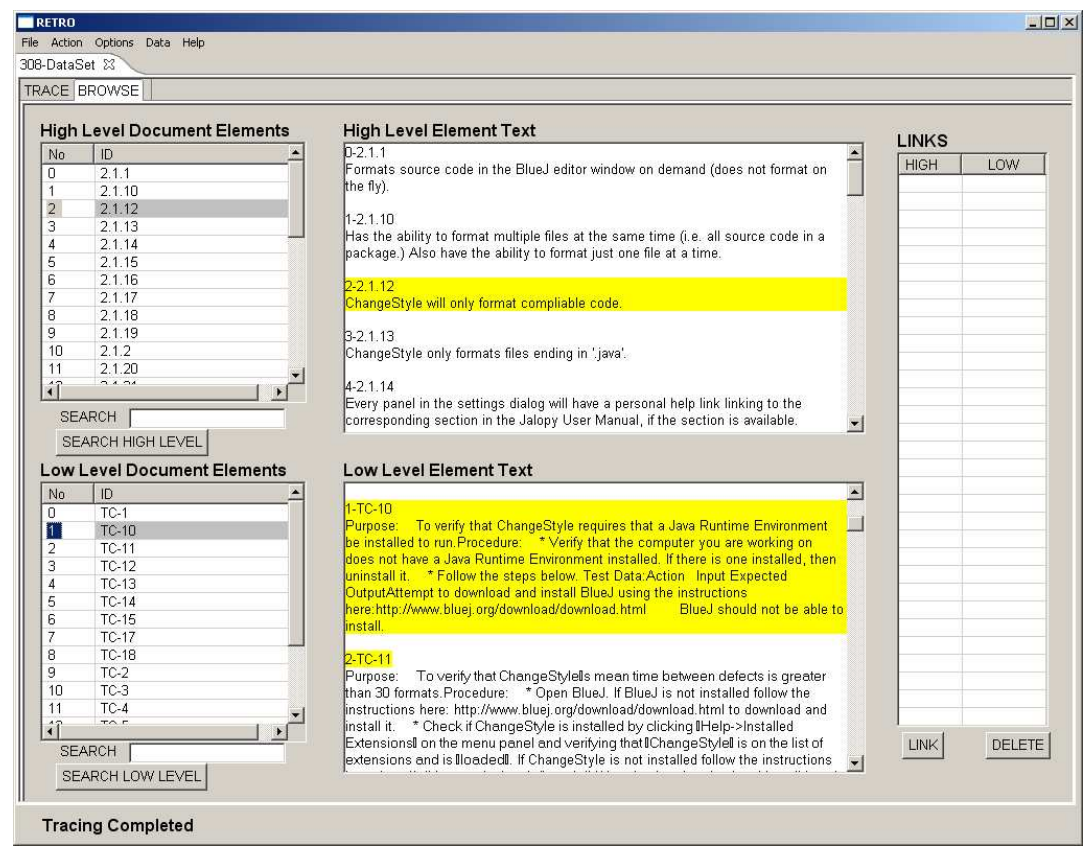

(b) RETRO's "browse" tab allows for analysts to view all links and add links IR methods may have missed.

Figure 4.2: Overview of the RETRO UI (a) shows the trace tab where (b) shows the free browse mode. 
methods in order to deliver pre-generated candidate TMs of specified precision and recall. In addition the IR toolkit and user feedback processing were excluded.

\subsubsection{RETRO.net tool}

RETRO.net was designed by Jody Larsen as a simplified and improved version of RETRO[11]. The UI for RETRO.net is displayed in figures 4.3(a), 4.4, and 4.3(b).

As seen in Figure 4.3 the UI provides similar capabilities as RETRO, only in a more consistent manner. The recommended links and all links tabs are very similar and give the analyst a uniform feel as contrasted to Figure 4.2. This tool was made to simplify the developer's job and make it easier for them to quickly learn the tool and understand how best to use it. In addition, RETRO.net was enhanced to allow keyword searches for both the high and low level elements. This keyword searching (for low level) is shown in Figure 4.4.

RETRO.net, similarly to RETRO, was designed to use vector space retrieval methods to suggest candidate TMs to an analyst. Unlike RETRO, which is written in both $\mathrm{C}++$ and Java, RETRO.net is written using $\mathrm{C} \#$ and the Microsoft .net framework. This makes the code much easier to understand and to maintain the entire system. In addition, as the entire RETRO.net system is written in one language, the IR methods and the UI can communicate directly without going through XML files on the hard drive. Similarly to RETRO, RETRO.netproduced final TM can be saved to the hard drive as an XML file indicating the links of the TM.

In addition, to allow us to better understand what the analysts did during their validation process an event logger was integrated in to RETRO.net. The 


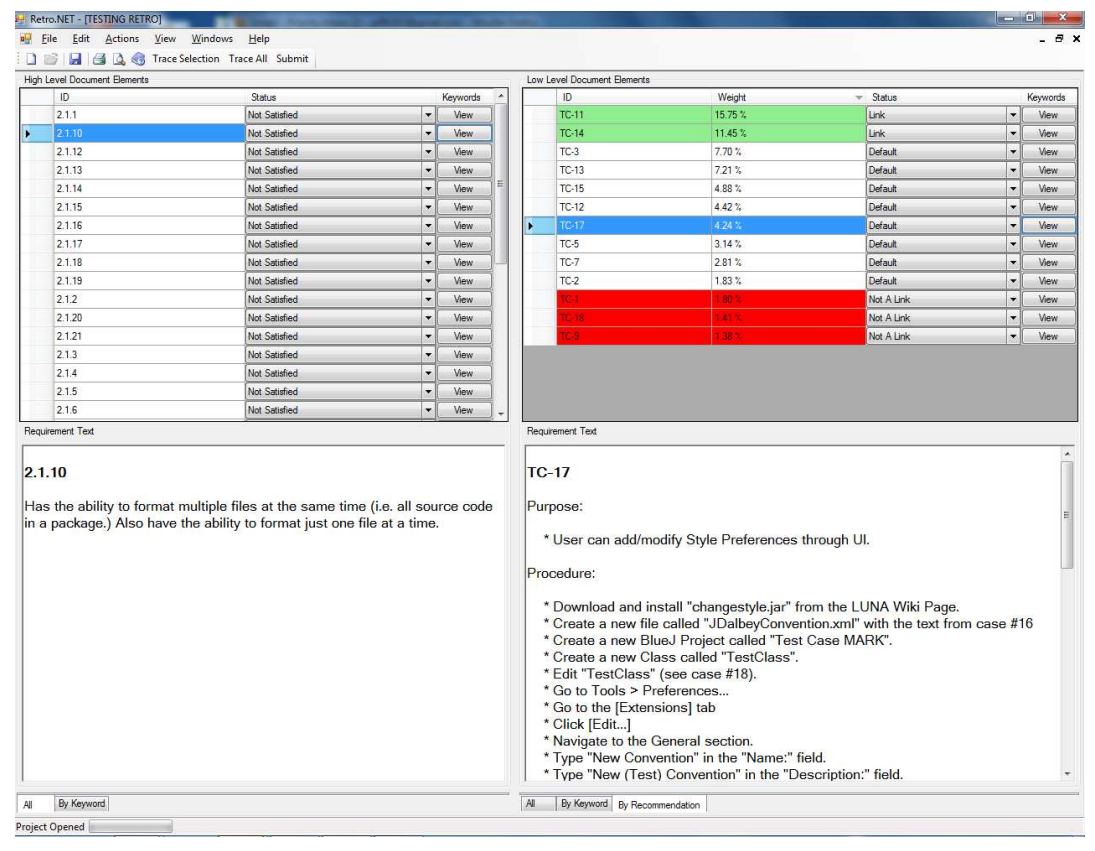

(a) RETRO.net's recommended links tab, displaying links the tool recommends.

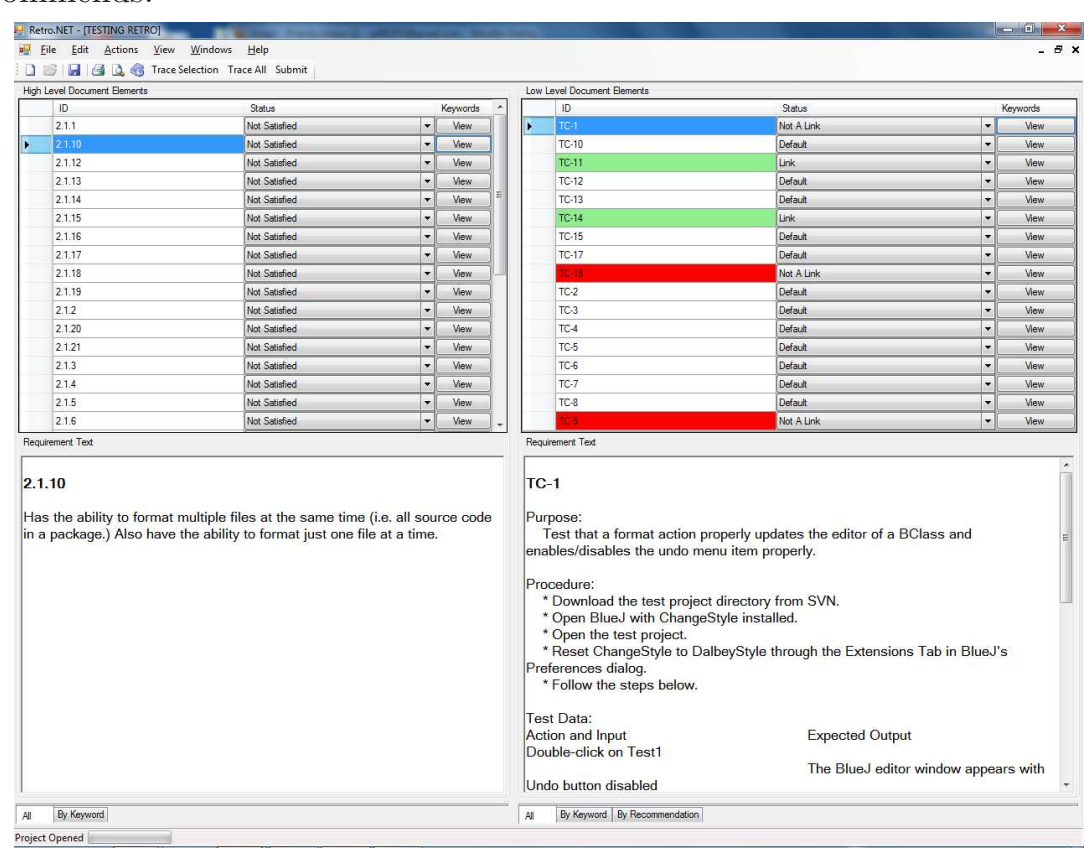

(b) RETRO.net's free browse tab, allowing analysts to view all potential links and add in links the tool may have missed.

Figure 4.3: RETRO.net's UI (a) shows the recommended links tab where (b) shows the free browse, all links mode. Also see figure 4.4 for the keyword search view. 


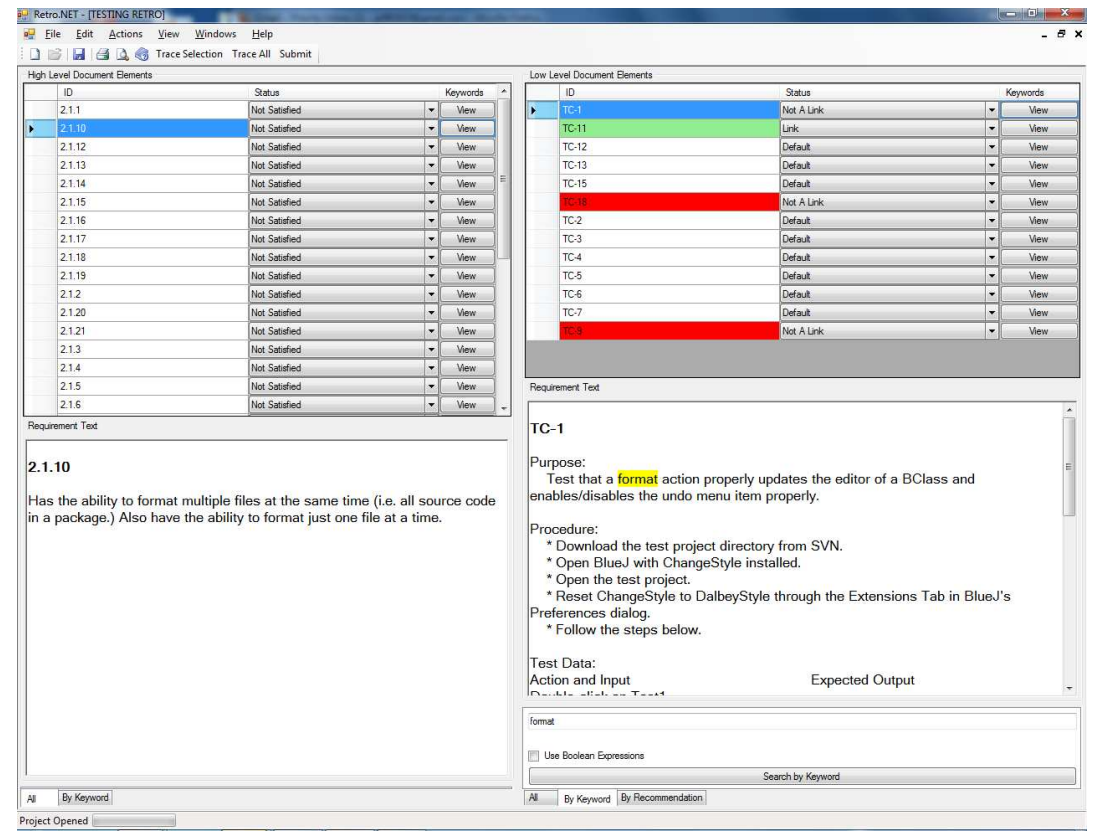

Figure 4.4: RETRO.net's keyword tab, allowing analysts to search low or high-level elements by keywords.

logger was created by Kong et al.[22] This logger tracked the following actions:

- User selects a source/target element in the TM.

- User views recommended links, views all links, or performs a keyword search (using the tabs at the bottom of the RETRO.NET UI window).

- User marks the observed source/target element pair as a (true) link or not a link.

- User marks a source element as satisfied, partially satisfied, or not satisfied by target elements.

This logging allowed us to simplify the participant tasks by allowing us to replace the manual log sheets with the auto-generated system log files.

As with Cuddeback et al.[6], we removed the IR back-end and added in a back-end that allowed the research team to have control over the precision and 
recall of the TM we provided to different participants. The new candidate TM delivery was tied to a userID. The TM is generated by starting with the TM generated by the RETRO.net IR methods and than adds or subtracts candidate links to achieve the desired precision/recall values. This is the same process used by Cuddeback et al. We were able to directly compare what happened to TMs with the same starting accuracy using different tools.

Further modifications, made to this tool to accommodate our second experiment, are described in Section 6.1.

\subsection{Experimental Design}

All the experiments were conducted with a similar protocol but with minor modifications to accommodate the specifics of the process used. What follows is an explanation of the overall protocol followed by sub-sections highlighting the key differences in the experimental design and setup.

The experiments were conducted at two different universities: Cal Poly and University of Kentucky. The studies were conducted during upper division and graduate-level software engineering and computer science courses. Participation was voluntary and students were offered, at the professors' discretion, extra credit for participating. The participants ranged from sophomores to graduate students.

All participants signed an informed consent form ${ }^{2}$, after which they were given a pre-study survey to obtain some demographic information as well as gauge their experience and comfort level with requirements tracing. After accepting these forms a researcher gave a short lecture on requirements tracing, its importance,

\footnotetext{
${ }^{2}$ Participants were required to accept this to participate.
} 
and what tools the participants would be using ${ }^{3}$.

For experiments utilizing a software tracing tool the researcher gave a quick walk through of the key functionality of the software tool. Following this, the participants were asked to download the software tool and practice tracing on the smaller practice dataset described in Section 4.3. During this practice session, participants were also given a hard copy sheet explaining the key functionality of the tool and how to use it to vet links. Participants were encouraged to complete the practice dataset vetting, however nothing was collected to ensure this was done.

After the pre-study surveys were collected, the research team examined them to determine the level of tracing experience for each participant. We made tracing experience a binary decision variable: either participants had prior tracing experience or not. This experience benchmark was used to ensure that the sampling was not impacted by assigning all experienced participants the starting TMs with similar accuracy (e.g high-precision and high-recall).

After this, each participant was assigned a unique userID which was tied to the starting TM accuracy expressed as precision and recall. Each userID was unique, tied to a specific TM and participant. However, TMs could be duplicated by assigning them to multiple userIDs.

Approximately one week following the training, the research team returned to administer the experiment. Participants were given a paper handout with the experiment directions (including the participants userID) (shown in Appendix D), a link to download the tool for software-based experiments, or hardcopies of the artifacts for the manual experiment (shown in Appendix H), a second copy

\footnotetext{
${ }^{3}$ This lecture was abbreviated in the manual study due to time constraints.
} 
of the software tool instructions, and instructions on how to submit their final TM via email for software experiments. Tracing occurred during a one hour class period, however participants were allowed to finish after class if they were not done by the end of the period. After the participants completed the tracing task they were instructed to email their final TM XML file and their log file (where applicable) to our research team email account. In the manual tracing experiment, participants submitted hard copy TM forms to the research group. A sample of one such completed form is shown in Appendix B. Upon receiving this, an auto-response to their email was generated directing them to also complete a post-study survey. The survey included questions on how much they used the software tool, how much they used specific features in the tool, how prepared they felt about tracing, their comfort level with tracing, and if they feel they would like to trace by hand or using the software in the future. This post-study survey is shown in Appendix F.

\subsubsection{Manual study}

Our manual study was set as a control to the other cohorts. We wanted to see the impact of asking an individual to trace a TM from hard copies rather than using a software tool. When giving the participants the TM we provided a document of all the requirements and a document of the test cases. In addition we provided the participants with a candidate TM by providing a list of requirement IDs and test IDs we mapped to them. To generate these lists we used the method described in Section 4.1.2.

During the tracing process we asked the participants to note all the links they considered as well as the links they confirmed on a trace form (this form is 
shown in Appendix B). While the trace form could have included the candidate links and had the analyst render decisions on these links, we choose to have this form be empty. This choice was in line with our other experiments where all analyst decisions must be explicit, i.e. the analyst must write the link decision versus merely crossing out incorrect links displayed. This trace form gave us a coarse-grained understanding of the tracing actions of participants in a minimally obtrusive way.

Upon completing the experiment participants were asked to hand in their manual trace form and were given a post-study survey to complete. Participants were informed the task was considered complete only after they had handed in their manual trace form and their post-study survey.

In this experiment no pre-study survey was included, however both pre- and post-study questions were included on the post-study survey.

\subsubsection{RETRO.net study}

The RETRO.net study followed the general procedure described in Section 4.2. Prior to the study, the participants were shown a demo of RETRO.net conducted by a member of the research staff. This was followed by all participants receiving the informed consent form, pre-study survey, and an overview of the RETRO.net tool. These forms are shown in Appendix A, E, and C. In addition, participants were given a download of the RETRO.net and the training dataset and asked to practice tracing using the tool. The research staff was available to answer any questions and assist the participants during this process.

After the pre-experiment surveys were collected the research team followed the process described in Section 4.2 to assign participants a userID tied to a 


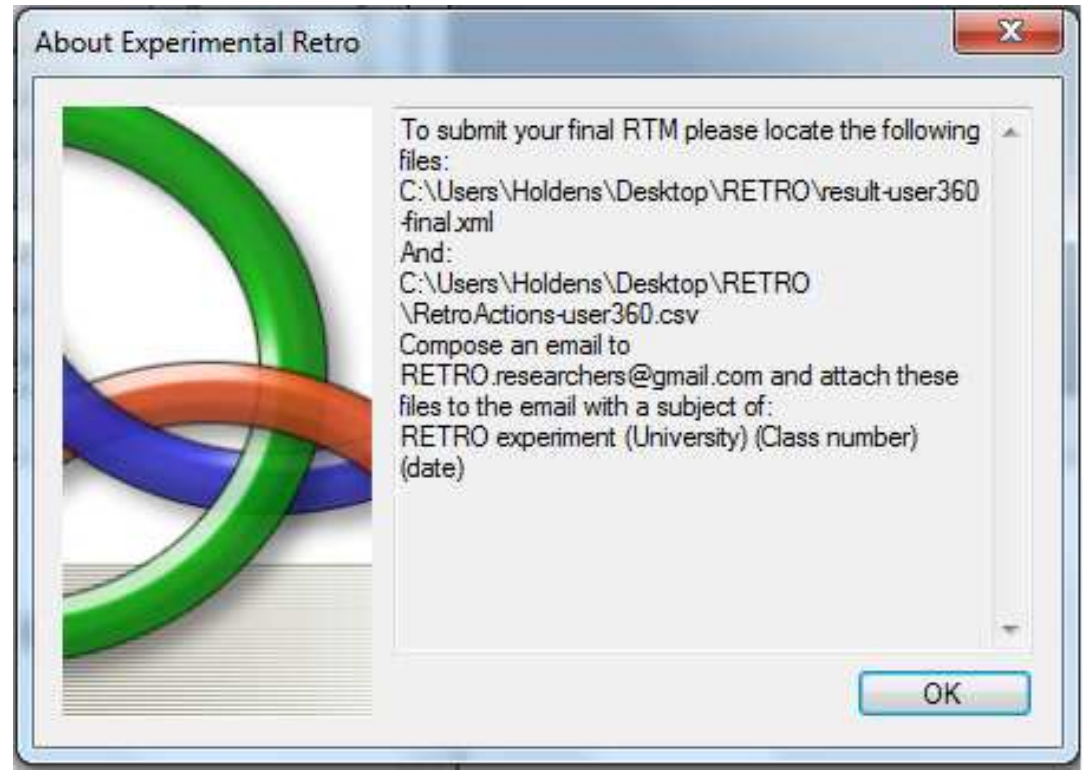

Figure 4.5: Submission instructions for participants using RETRO.net

precision-recall value pair.

The following week the research team returned to conduct the main tracing task. During the experiment, participants were given the experiment instructions (shown in Appendix D) as well as the RETRO.net tool documentation. Upon completing the task, RETRO.net instructed participants how to submit their results to the research team. The submission instructions are shown in Figure 4.5. This process involved participants locating their final TM XML file and their action log file (a csv file described in 4.1.2) and attach these files to an email to the research team mailbox. This mailbox was set up to return an auto-response to the participants requesting they complete a short post-study survey. This survey was an online survey with questions on how much they used the software tool between the training and experiment, how much they used specific tool features, how prepared they felt about tracing, their comfort level with tracing, and if they would prefer tracing by hand or using a software tool in the future. The full post-study survey is shown in Appendix F. 


\subsection{What we Traced}

The Cuddeback et al.[6] and our RETRO.net study utilized two datasets, a smaller training dataset and a larger dataset, ChangeStyle. For the manual experiment we only utilized the larger dataset as their was no formal training period. These datasets are described below:

\subsubsection{Training Dataset}

The training dataset, which is used in software-based experiments to familiarize the participants with the use of RETRO or RETRO.net. This dataset describes the requirements and test cases for a very small program for a moonlander game. The dataset comes from a programming assignment used in introductory computer science courses to familiarize the students with programing. The training dataset is small, consisting of 10 requirements and 5 test cases. The full dataset is shown in Appendix G.

\subsubsection{ChangeStyle Dataset}

For the actual experiment we utilized ChangeStyle, the dataset utilized by Cuddeback et al. This dataset is a pair of artifacts, requirements and test cases, for a BlueJ plugin which formats Java code. This dataset contains 32 requirements, 17 test cases, and 24 true-validated links.

This dataset originated from a two-quarter project from a junior-level software engineering course sequence. We selected this project because we wanted to ensure all participants would easily understand the material. This was a crucial point as we wanted to determine how analysts who would be familar with doc- 
uments and subject matter would interact with the tracing system, rather than test comprehension of the documents themselves.

This dataset consists of student-generated artifacts, making it feasible for the participant to read all requirements and gain an understanding of the system. In addition, the content of the dataset dealt with Java code style-formatting. This is a topic that is covered in many introductory software engineering courses. This aided the overall participant familiarity with the subject.

An additional important consideration when choosing this dataset was that the tracing task must be achievable in a small time frame. We needed the participants to be able to complete the validation task in a period of about one hour (the length of a course lab period). Additionally, as reported in Egyed et al. analysts reach optimum performance in validation tasks in less than 60 minutes[13]. We wanted to focus on optimal analyst tracing behavior and not introduce analyst fatigue. This dataset was small enough to allow the task to be accomplished within this optimal time frame without being so small it would be trivial.

Finally, this dataset was utilized by Cuddeback et al.[6]. By utilizing the same dataset in our follow up experiments we could directly compare and analyze our results to the those presented by Cuddeback et al.

The true "golden standard" TM, which we use to measure the precision and recall of TMs, originated from the students who built the dataset. The TM was then vetted by Cuddeback et al. who created a final TM[6]. When extending the RETRO study we conducted an additional validation of the golden standard. We located several potential changes of the golden standard. In order to obtain an accurate golden standard we discussed these changes with several members of our research group and reached a consensus for any changes. The links we 
considered, our discussion, and ultimate decisions were all documented. With several research members verifying the golden standard we feel confident that we have the correct TM. While the golden standard is not included due to its use in on-going experiments, we do make it available upon request from other research groups for use or verification.

\subsection{Information Collected}

In the experiments we collected a rich set of meta-information from surveys as well as information concerning initial and final TMs for each analyst. This information collection started with Cuddeback et al.[6], however Cuddebeback did not perform analysis on most of this information. We separate these variables into three categories:

- Baseline independent variables(Table 4.2): these variables specify the accuracy of the initial TMs. These are the variables the research team directly controlled.

- Observed independent variables(Table 4.3): these variables contain information about the experiment participants and their work on the tracing task. This information was either part of the experimental design (location, software used) or collected from the pre- and post-study surveys. Of the 11 variables collected, one (Time) is continuous; the remaining 10 are either nominal or ordinal.

- dependent variables(4.4): these variables measure the accuracy of the final TMs submitted by the participants. These variables fall into two groups: measure of the absolute accuracy of the final TM and "Delta" 
Table 4.2: Baseline Independent Variables

\begin{tabular}{|l|c|r|}
\hline Variable & Abbreviation & Scale \\
\hline Initial Precision & SPrec & {$[0,1]$} \\
\hline Initial Recall & SRec & {$[0,1]$} \\
\hline Initial $f_{2}$-measure & SF2 & {$[0,1]$} \\
\hline Initial Quadrant & SQuadrant & $\{Q 1, Q 2, Q 3, Q 4\}$ \\
\hline
\end{tabular}

Table 4.3: Observed Independent Variables

\begin{tabular}{|l|c|c|c|}
\hline Variable & Abbreviation & Scale & Type \\
\hline Procedure used & Procedure & $\begin{array}{c}\{\text { Retro, Manual, } \\
\text { RETRO.net }\}\end{array}$ & Nominal \\
\hline Location & Location & $\{\mathrm{CP}, \mathrm{UK}\}$ & Nominal \\
\hline Software Engineering Experience & SEExp & $\{0,1,2\}$ & Ordinal \\
\hline Tracing Experience & TRExp & $\{0,1\}$ & Nominal \\
\hline Time to preform tracing task & Time & $\#$ minutes & Cont. \\
\hline Grade Level & Grade & $\{\mathrm{F}$, Soph, J, S, G $\}$ & Nominal \\
\hline Confidence with tracing & TrConf & $1-5$ & Ordinal \\
\hline Opinion on Tool vs. Manual & Opinion & $\{$ Man, SW $\}$ & Nominal \\
\hline Effort on searching for omitted links & MissingEff & $0-5$ & Ordinal \\
\hline Effort on validating offered links & ValidEff & $0-5$ & Ordinal \\
\hline How prepared the analyst felt & Prepared & $1-5$ & Ordinal \\
\hline
\end{tabular}

variables that measure the change between the initial and final TMs.

What follows is a description of each variable and where we gathered this data from.

Table 4.4: Response (dependent) variables

\begin{tabular}{|l|c|c|}
\hline Variable & Abbreviation & Scale \\
\hline Final Precision & FinPrec & {$[0,1]$} \\
\hline Final Recall & FinRec & {$[0,1]$} \\
\hline Final $f_{2}$-measure & FinF2 & {$[0,1]$} \\
\hline Change in Precision & $\Delta P r e c$ & {$[-1,1]$} \\
\hline Change in Recall & $\Delta R e c$ & {$[-1,1]$} \\
\hline Change in $f_{2}$-measure & $\Delta F 2$ & {$[-1,1]$} \\
\hline
\end{tabular}


Table 4.5: Response (dependent) variables

\begin{tabular}{|l|c|c|}
\hline Quadrant & Precision & Recall \\
\hline QI & $<50 \%$ & $<50 \%$ \\
\hline QII & $<50 \%$ & $\geq 50 \%$ \\
\hline QIII & $>50 \%$ & $\geq 50 \%$ \\
\hline QIV & $\geq 50 \%$ & $\geq 50 \%$ \\
\hline
\end{tabular}

\subsubsection{Baseline Independent Variables}

The baseline variables are initial precision, initial recall, initial $f_{2}$-measure, and initial quadrant. The precision and recall were both assigned by the research team to each analyst. The initial $f_{2}$-measure is computed using the precision and recall values.

The quadrant is a variable we created to separate the precision-recall space into four regions. The quadrant breakdown is shown in Table 4.5.

In addition, we recorded the number of hits (or correct links), number of misses (or false positives), and the sum of these two numbers which is the size of the initial candidate TM. Given the size of the true TM, the number of hits and misses are uniquely determined by the initial recall and precision.

\subsubsection{Observed Independent Variables}

The following is a description of each observed independent variable and an explanation of where this data was obtained.

1. Procedure used: Specifies which experiment the participant was in. The possible values are RETRO (for Cuddeback et al.), manual, or RETRO.net. 
2. Location: Which location the participant was located at, either Cal Poly or University of Kentucky.

3. Software Engineering experience: A measure of a participant's familiarity with software engineering on a 3 point scale $(0,1,2)$. This was determined by looking at question 3 (software courses taken) and question 5 (industry experience) on the pre-study survey. The value was calculated as follows: 1 point for $2+$ software courses and 1 point for industry experience.

4. Tracing experience: A measure of the participant's familiarity with requirements tracing. This variable was set to "yes" or "no" using the following input: Question 4(TM generation in courses), question 6 (industry tracing experience), and question 10 (how many time performing tracing). The analyst was given experience if they marked 2 or more courses or had industry tracing experience and had preformed tracing more than 2 times. In total we recorded 18 participants with tracing experience.

5. Time to preform tracing task: A measure of how long (in minutes) the participant took to complete their tracing task. We obtained this value as a self-reported time, in RETRO and the manual experiment, and using the generated logs for the RETRO.net experiment.

6. Grade level: The participant's self-reported grade level in school (e.g. junior, senior, etc.). This was recorded as question 2 on the pre-study survey.

7. Confidence in tracing: Self-reported by participants. While we collected both their confidence in tracing prior to the experiment (question 7 prestudy) and post (question 21 post-study), we chose to analyze the poststudy survey response as we felt this would be the best indicator in overall 
comfort $^{4}$. In addition, we chose this to maintain consistency as the manual experiment did not take a pre-study survey, only post-study survey.

8. Opinion on Tool vs. Manual: This was a new question added to the survey for the manual and RETRO.net experiments. This was asked postexperiment to gauge comfort levels and individual preferences. This data was gathered in post-experiment survey question 22 .

9. Effort on searching for omitted links: This was self-reported and was a gauge on how often the participant went searching for omitted links. This data was obtained in the post-experiment survey question $6^{5}$.

10. Effort on validating offered links: This was self-reported and was a gauge on how often the participant attempted to validate links in their given candidate TM. This data was obtained in the post-experiment survey question $7^{5}$.

11. How prepared the analyst felt: This was the self-reported analyst feeling of preparedness. This data was obtained in the post-experiment survey question 20 .

\subsubsection{Dependent Variables}

The dependent variables are those that measure the final TM accuracy. This includes both absolute accuracy and change in accuracy. The absolute accuracy variables are:

\footnotetext{
${ }^{4}$ This assumption was confirmed as only 4 participants in all experiments reported a lower comfort level post-experiment as compared to pre-experiment

${ }^{5}$ Questions were formulated differently for different experiments, however in each case they referred to the same set of activities.
} 


\section{Final Precision}

\section{Final Recall}

\section{Final $f_{2}$-measure}

The change in accuracy variables which measure the change from initial to final TM accuracy are:

1. Delta Precision $=$ Final Precision - Initial Precision

2. Delta Recall $=$ Final Recall - Initial Precision

3. Delta $f_{2}$-measure $=$ Final $f_{2}$-measure - Initial $f_{2}$-measure

In the study conducted by Cuddeback et al. the main focus was on how the baseline variables impact the dependant variables (although no statistical analysis was presented)[6]. We present formal statistic results for both baseline and observed variables and their impact on the dependent variables. These results are presented in Section 5.5.

\subsubsection{Research Questions}

To assist in answering our overall goals as discussed in Section 3 we broke down each goal. Specifically we desired to answer the following questions:

- Is the effect of the accuracy of the initial TM on the accuracy of the final TM statistically significant? 
- Are the effects of any observed independent variables on the accuracy of the final TM statistically significant (when controlled by the initial TM accuracy)?

- Which group of independent variables has a higher effect on the accuracy of the final TM: the baseline independent variables or the observed independent variables?

While Cuddeback et al. provided observations for how the baseline independent variables impacted the dependent variables, they did not support any of their findings with statistical analysis. By answering these questions we look to fill in some of the gaps left by Cuddeback et al. and gain a more in depth understanding about how analysts interact with semi-automated tracing systems. 


\section{Chapter 5}

\section{Results for Artificially Generated TM experiment}

In total we conducted and collected data for two experiments and preformed data analysis on the three cohorts. These included the RETRO.net study (2 locations) and the manual study (1 location). In addition we included data from the Cuddeback et al. study[6] in our data analysis. Table 4.1 gives an overview of all experiments. This table also includes the Cuddeback et al. experiment (shown as the RETRO experiment).

\subsection{RETRO}

We include a summary of the experiments presented by Cuddeback et al.[7, 6] In these experiments 33 participants responses were collected. The starting points for all participants is shown in Figure 5.1(a). Each point in this figure represents the precision and recall of the participants initial candidate TM. 
Additionally Figure 5.2(a) shows how each participant performed. In this figure each participant is represented by a vector where the tail of the vector is the participant's initial TM accuracy and the head is their final submitted TM's accuracy.

\subsection{RETRO.net}

This experiment was conducted at Cal Poly and University of Kentucky. We collected eight responses from one university and five from the other giving a total of 13 participants. As discussed in section 4.2, we split the cohort into two groups, experienced and non-experienced. We determined four participants were experienced and nine were un-experienced. The starting TMs assigned to participants are shown in Figure 5.1(b). Each point in this figure represents the precision and recall of the participants initial TM. This figure shows an uneven distribution of candidate TMs. The study initially involved more participants at each site. The distribution was caused by non-response from several participants.

Figure 5.2(b) shows how participants performed. Each participant is represented by a vector where the tail is the vector is the participants initial TM and the head is their final TM submitted.

\subsection{Manual study}

The manual study was only conducted at Cal Poly in two sections of a juniorlevel software engineering course. In total we had 38 participants who were all junior or senior standing. The starting TMs assigned to participants are shown in Figure 5.1(c). As discussed in 4.2.1 the participants were asked to trace manually 


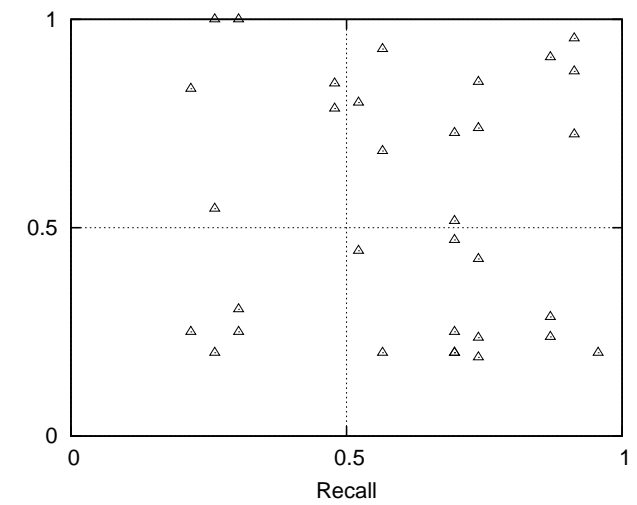

(a) initial TMs for Cuddeback et al. study

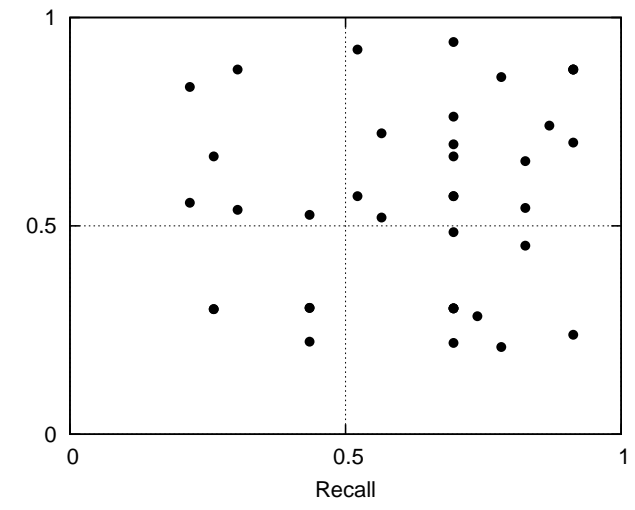

(c) initial TMs for the manual study

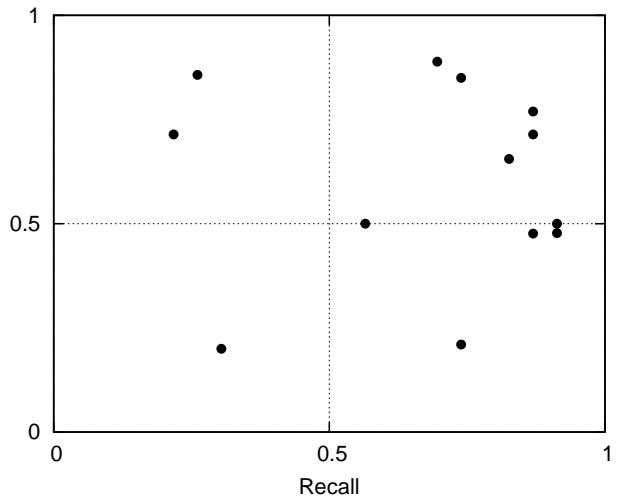

(b) initial TMs for RETRO.net study

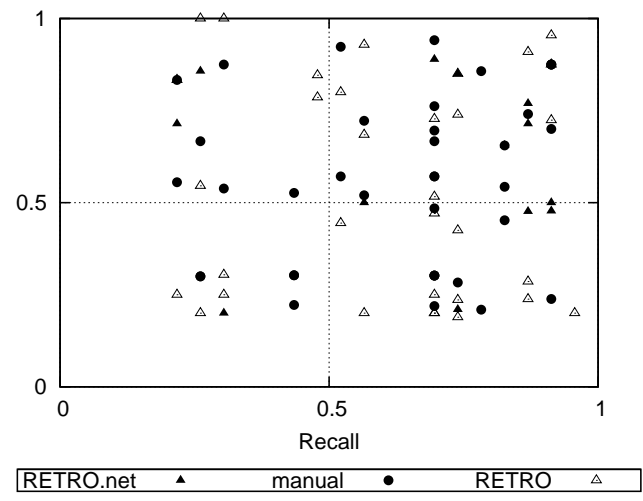

(d) initial TMs for all three studies

Figure 5.1: Starting TM in precision and recall space for (a) Cuddeback et al., (b) RETRO.net, (c) manual, and (d) all studies

without software assistance.

Figure 5.2(c) shows how participants performed when vetting manually.

\subsection{Observation Analysis}

In the study conducted by Cuddeback et al. made several important observations. Three of the key observations were the tendecy for analysts to drift towards the precision $=$ recall line, different analyst behaviors for different initial TM regions, and the lower initial TM $f_{2}$-measure, the larger the change in $f_{2^{-}}$ 

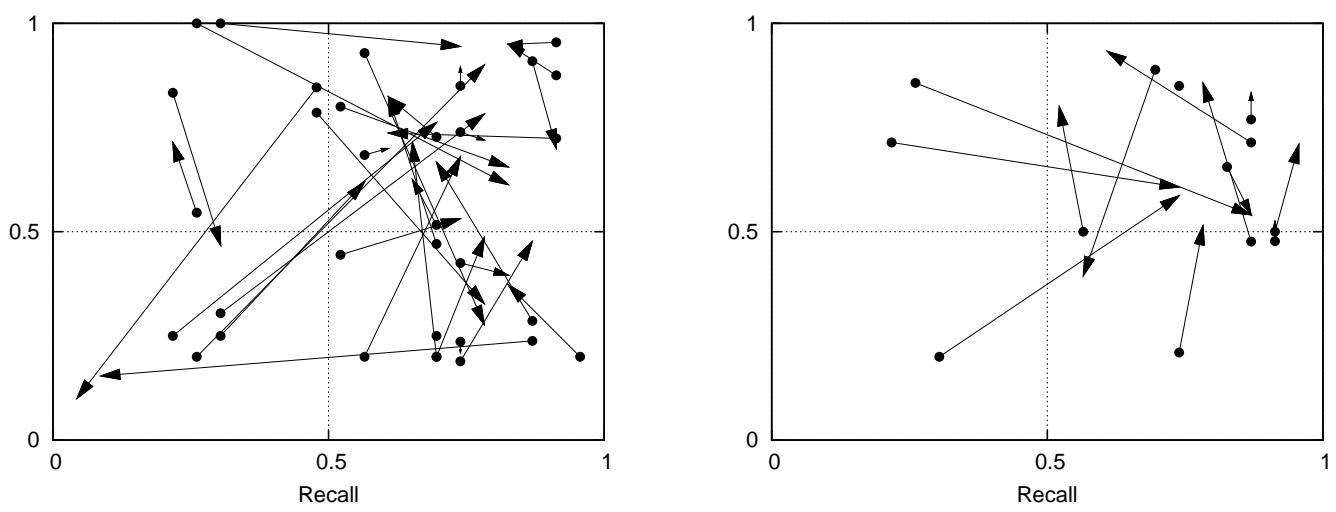

(a) Drift in precision-recall from initial to final (b) Drift in precision-recall from initial to final TM for the Cuddeback et al. study

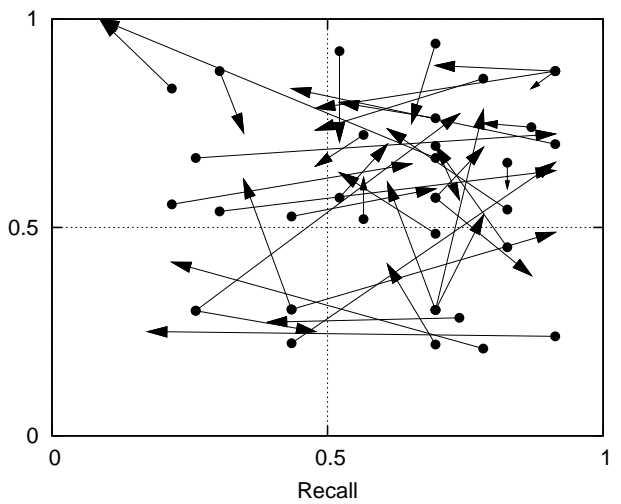
TM for the RETRO.net study

(c) Drift in precision-recall from initial to final (d) Drift in precision-recall from initial to final TM for the manual study

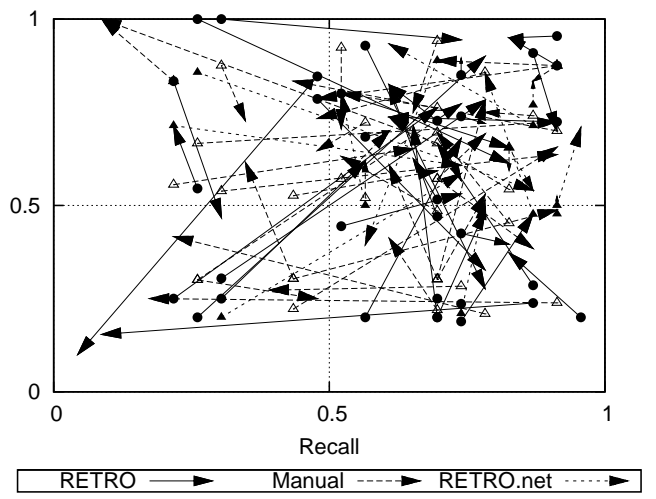

Figure 5.2: Change in precision and recall from initial to final TMs for the (a) Cuddeback, (b) RETRO.net, (c) manual, and (d) all three studies

measure for the final submission. Each of these observations is described below and we compare and contrast our extended dataset to their results.

\subsubsection{Movement towards recall $=$ precision}

In Cuddeback et al. one observation that was made was that analysts tended to move their TMs accuracy towards the recall = precision line. Cuddeback noted that this line represents TMs that have the same number of links as the true TM. It was conjectured that analysts have an intuition about the expected 
size of the TM based on the sizes of the artifacts they are tracing[6]. It was further suggested that the anticipated TM size would be between 17 to 32 links, the former being the number of requirements and latter being the number of test cases.

In Cuddeback et al. the observation was supported by the analyst performance. In the RETRO study, $62 \%$ of analysts produced final TMs in between this anticipated size range. This is visualized in Figure 5.3(a) where we plot the size of the final TM vs. the size of the initial candidate TM.

For both our manual and RETRO.net experiments we examined the final TMs for similar results. This shift from initial to final TM size is shown in Figure 5.3(b) for manual and Figure 5.3(c) for RETRO.net. As can be seen in these figures analysts in the manual and RETRO.net experiment did not fall in this range at as high of a rate as Cuddeback et al. Still 53\% (27 of 51) of the analysts fell in this range. Additionally, if we slightly expand this range (by 10\%, from 16 - 35 links), we increase this percentage to 67\% (34 of 51) of analysts. This range increase can easily be justified in that some requirements can easily trace to multiple test cases, resulting in slightly larger TMs. In addition, while not all analysts in these studies fell within this range, it should be noted that only three participants in either the RETRO.net or manual study submitted final TMs with more than 40 links.

In the manual study we did see two participants exhibit outlier behavior, submitting final TMs with very few final links. One participant received a TM with six links and reduced it to two links. This analyst focused only only eliminating false positives from the candidate links presented and did not search for missing links. The second analyst was more severe, starting with 24 candidate links and rejecting all but two. Similarly to the previous analyst, they only rejected can- 
didate links and did not search for any errors of omission. We feel, based on this behavior, that these participants are indeed outliers and did not truly understand the full task.

These results show that using this dataset, analysts have a concept of how large the TM should be and make their decisions based on this "ideal" size. This is reinforced when combining all the experiments together as shown in Figure 5.3(d). In this figure there is a clear clustering of the majority of submitted TMs in the region containing between 17 and 32 links with several just outside this region.

\subsubsection{Regional Behaviors}

Cuddeback et al. observed distinctly different behavior for participants with candidate TMs in different regions. These regions were low precision-low recall, low precision-high recall, high precision-low recall, and high precisionhigh recall. Each of these regions were separated by the $50 \%$ precision-recall lines.

Overall through both the manual and RETRO.net experiment we noticed similar behaviors from analysts for each of these regions. Figure 5.4(a) shows the average performance of analysts in each of the regions for Cuddeback's RETRO experiment. We show an average quadrant performance for the manual and RETRO.net experiments in Figures 5.4(b) and 5.4(c) respectively. Also, we combined all three studies together and show the average for each region in Figure 5.4(d). As can be seen in these figures, similar analyst trends were observed from each region.

Each region and the behavior observed, both for Cuddeback et al.[6], the 


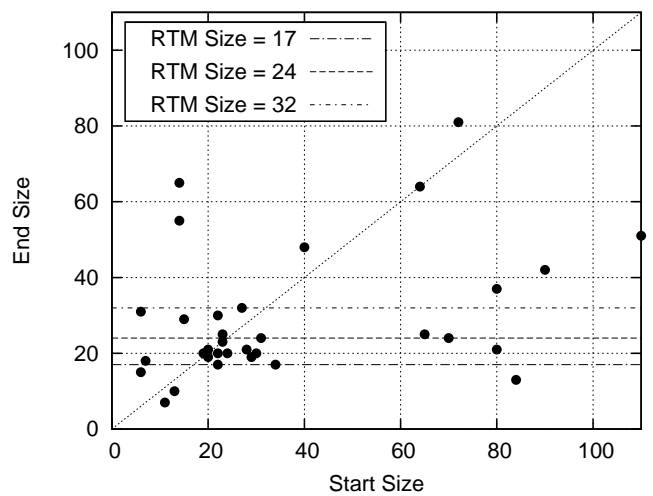

(a) Change in TM size for the RETRO experiment conducted by Cuddeback et al.[6]

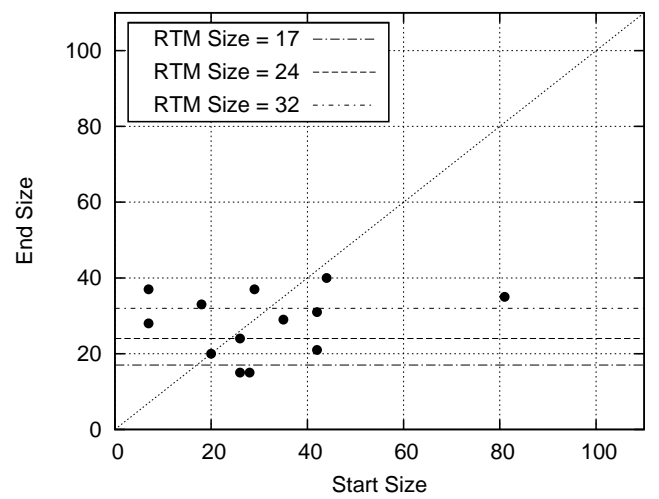

(c) Start-End TM size for the RETRO.net study

Figure 5.3: Change in the size of the TM from initial to file for the (a) Cuddeback, (b) manual, (c) RETRO.net, and (d) all three studies

manual experiment, and the RETRO.net experiment are examined in further detail below:

Low Precision, Low Recall: Cuddeback et al. observed participants improving both precision and recall substantially. These participants demonstrated the highest improvement in accuracy, as observed by delta $f_{2}$-measure. The analyst performance for Cuddeback et al. is shown in Figure 5.5(c).

When we examine how analysts performed in this region in both the manual experiment and RETRO.net similar trends are apparent. In fact, when looking at 


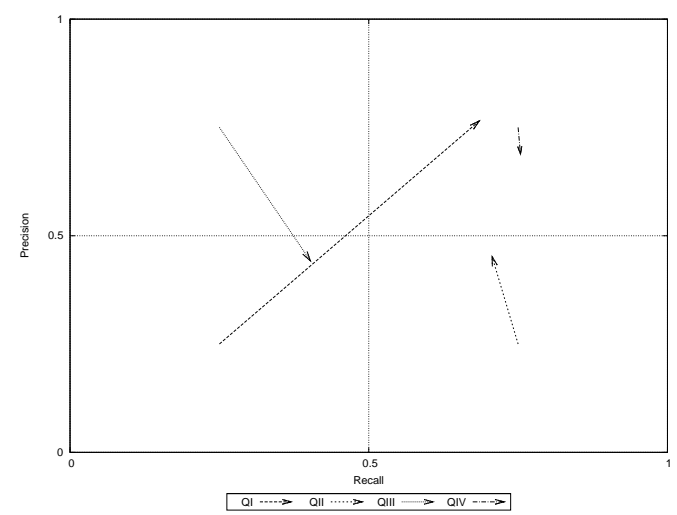

(a) Average $\Delta$ precision-recall by regions, Cuddeback et al. study[6]

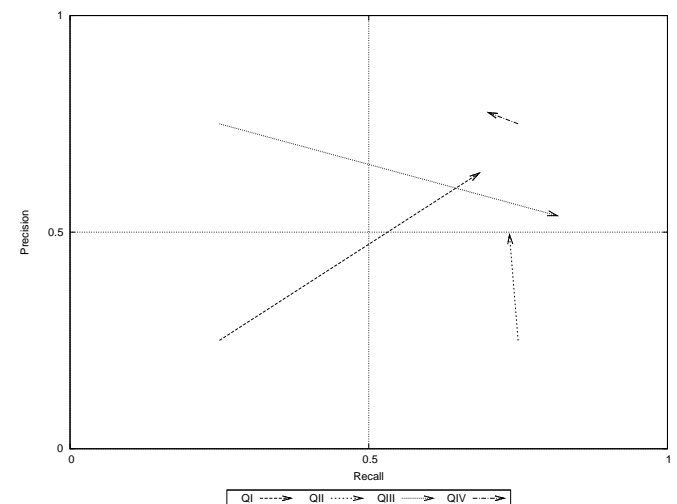

(c) Average $\Delta$ precision-recall by regions, (d) Average $\Delta$ precision-recall by regions, all RETRO.net study

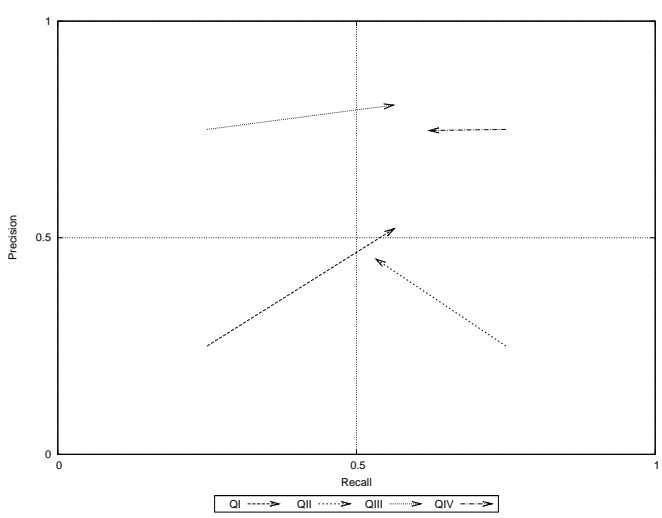
ual study

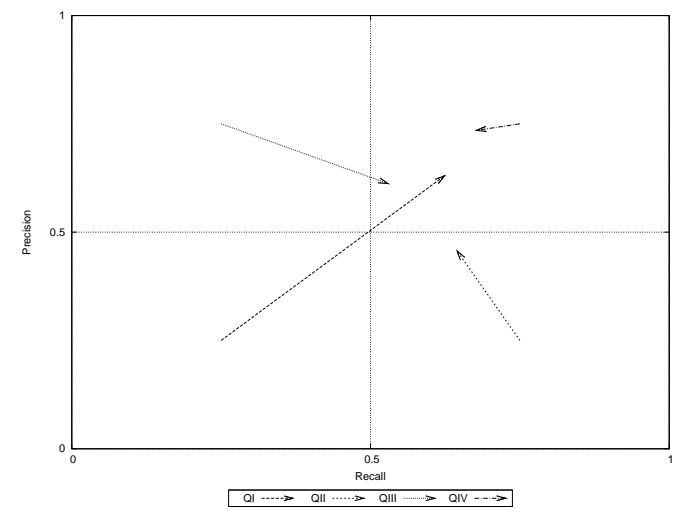

studies combined (b) Average $\Delta$ precision-recall by regions, man-

Figure 5.4: Average analyst performance for each of four regions in the (a) Cuddeback, (b) manual, (c) RETRO.net, and (d) all three studies

both experiments only 2 participants in the manual experiment did not improve both precision and recall. In addition, when examining the overall change in accuracy (as measured by $f_{2}$-measure) only one analyst did not improve and even then they only decreased $f_{2}$-measure by $2 \%$. In fact, when observing the delta $f_{2}$-measure from this region the participants improved their accuracy by 31\%. The performance of these participants is shown in Figure 5.6(c) for manual and 5.7(c) for RETRO.net. 

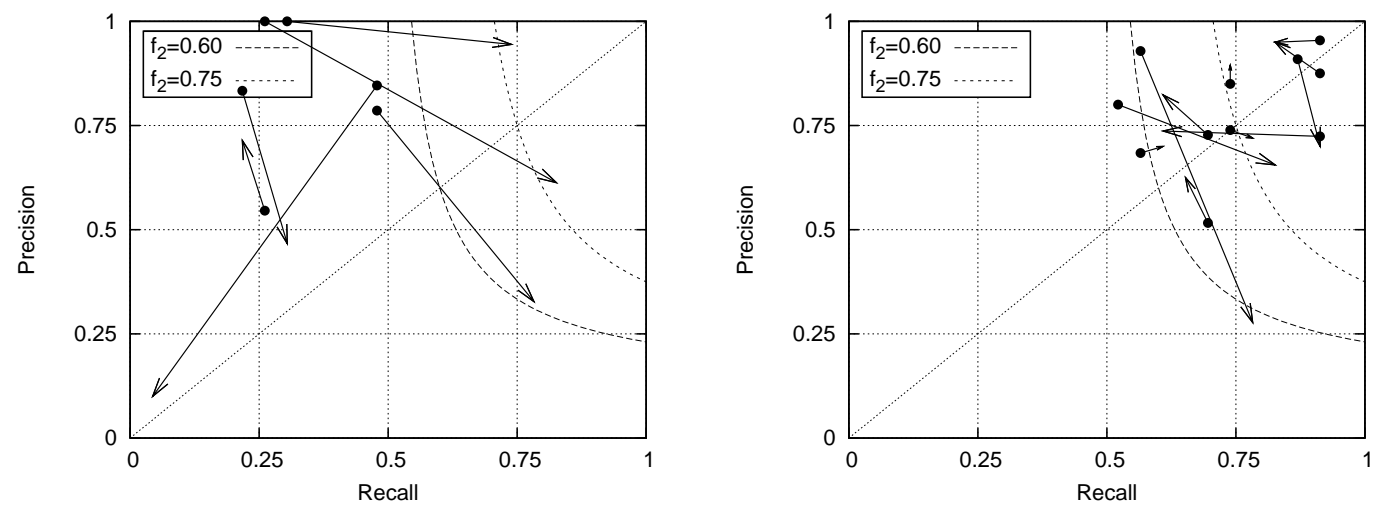

(a) Analyst behavior from Cuddeback's study, (b) Analyst behavior from Cuddeback's study, high-precision, low-recall area high-precision, high-recall area
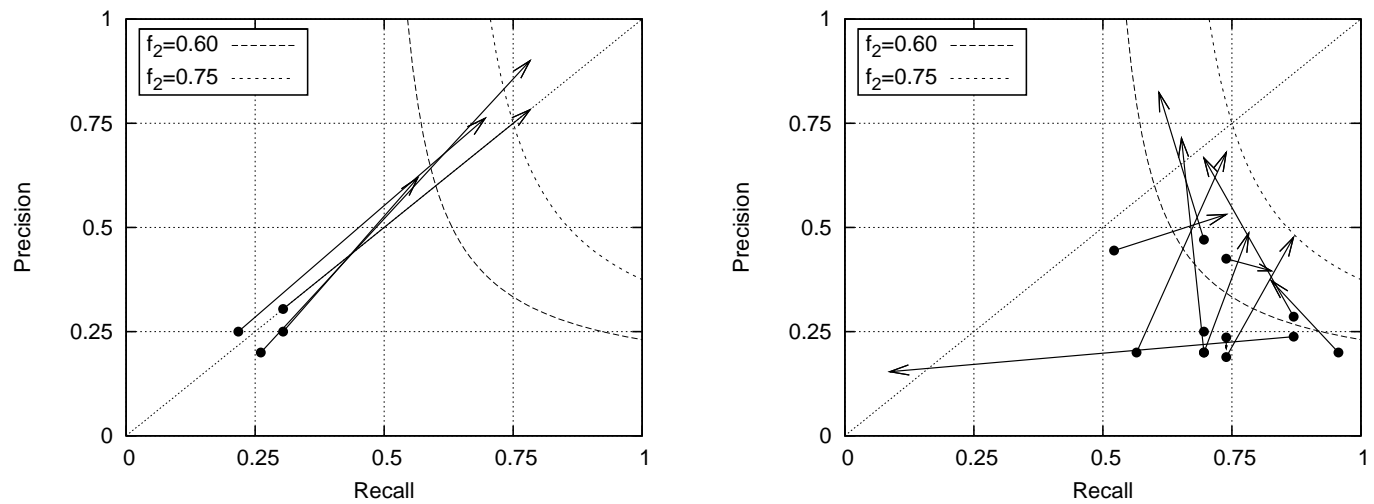

(c) Analyst behavior from Cuddeback's study, (d) Analyst behavior from Cuddeback's study, low-precision, low-recall area

low-precision, low-recall area

Figure 5.5: Analyst performance for each of four regions in the RETRO study: (a) Higha-precision, Low-recall; (b) High-precision, High-recall; (c) Low-precision, Low-Recall; and (d) Low-precsion, High-recall

Low Precision, High Recall: Cuddeback et al. observed that participants improving precision of the submitted TM, but generally decreasing recall. Cuddeback et al. conjectured that as TMs in this region have significantly more candidate links with a large percentage of false positives, analysts concentrate on determining errors of commission (false positives) without concentrating on locating errors of omission (links not in the candidate TM)[6]. This region's results for Cuddeback is shown in Figure 5.5(d).

In the manual and RETRO.net experiments this trend continued. The major- 

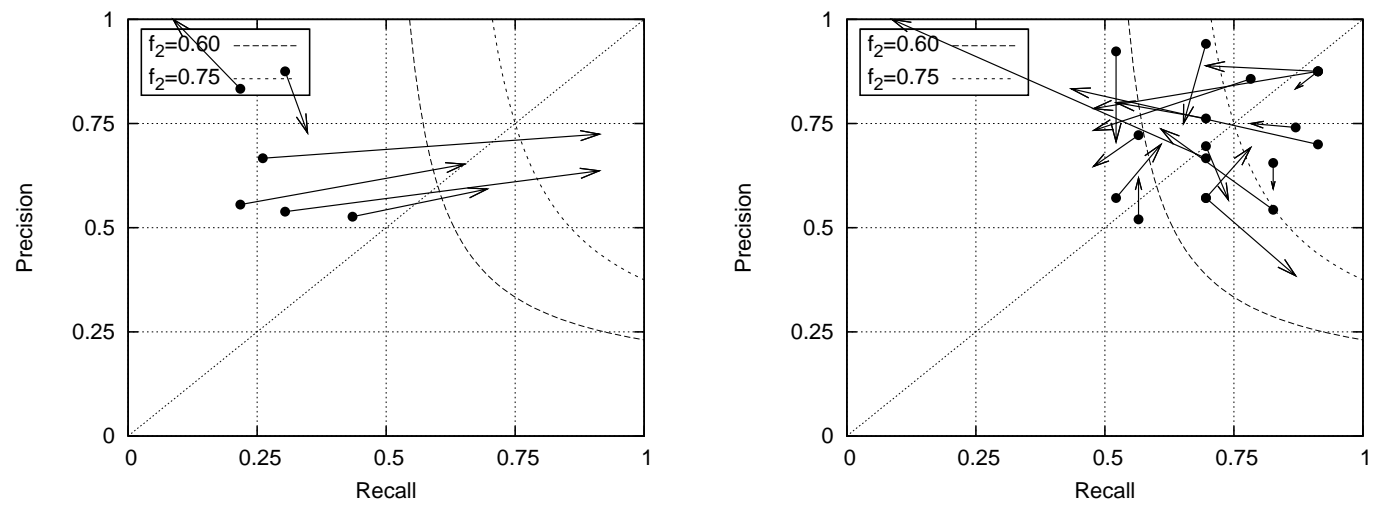

(a) Analyst behavior from the manual study, (b) Analyst behavior from the manual study, high-precision, low-recall area high-precision, high-recall area
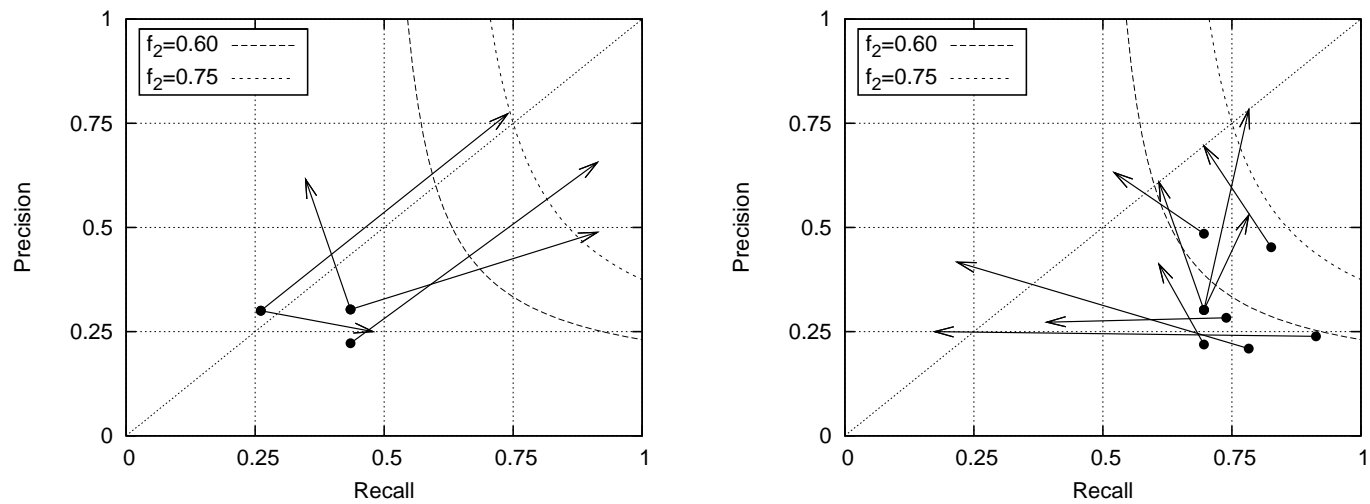

(c) Analyst behavior from the manual study, (d) Analyst behavior from the manual study, low-precision, low-recall area

low-precision, low-recall area

Figure 5.6: Analyst performance for each of four regions in the manual study: (a) Higha-precision, Low-recall; (b) High-precision, High-recall; (c) Low-precision, Low-Recall; and (d) Low-precsion, High-recall

ity of participants $(66 \%)$ improved precision but at the cost of decreasing recall. $25 \%$ of the analysts from this region improved both precision and recall, however their increase in recall was small between 4 and $8 \%$. There were also several participants who greatly decreased their recall with out increasing the precision. In the region were initial TM precision was less than $25 \%$ and the recall was greater than $75 \%, 3$ out of 4 participants exhibited this behavior. These participants were assigned the largest TMs in all experiments with greater than 80 initial links. When Cuddeback et al. originally observed this behavior it appeared 


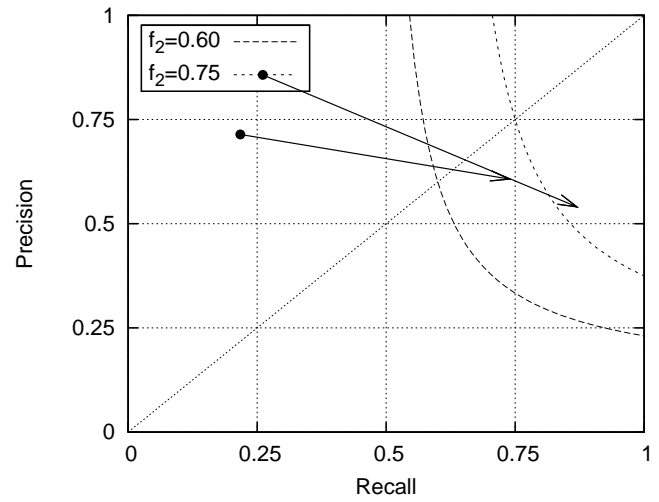

(a) Analyst behavior from the RETRO.net study, high-precision, low-recall area

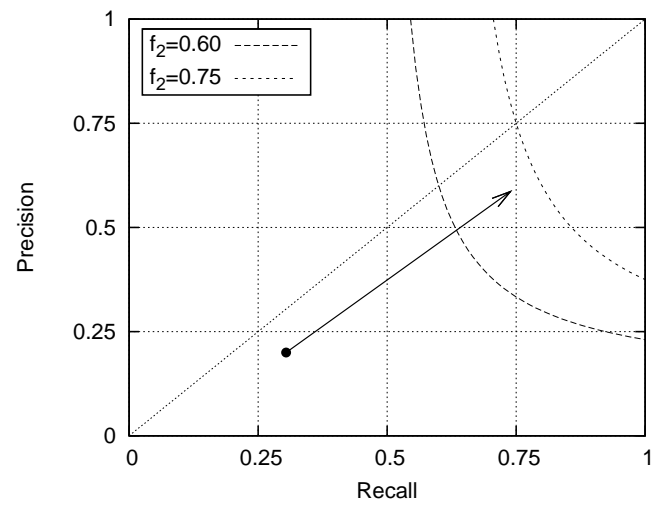

(c) Analyst behavior from the RETRO.net study, low-precision, low-recall area

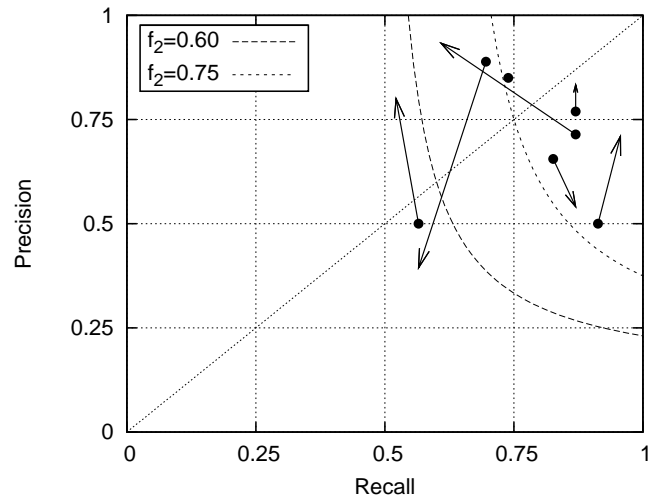

(b) Analyst behavior from the RETRO.net study, high-precision, high-recall area

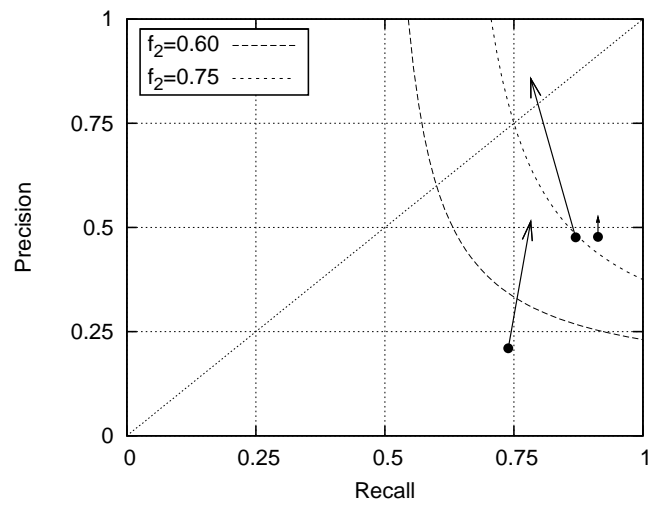

(d) Analyst behavior from the RETRO.net study, low-precision, low-recall area

Figure 5.7: Analyst performance for each of four regions in the RETRO.net study: (a) Higha-precision, Low-recall; (b) Highprecision, High-recall; (c) Low-precision, Low-Recall; and (d) Lowprecsion, High-recall

likely to be an outlier. However, with multiple participants from this region reproducing this behavior it should be asked why this is occurring. This region's behavior is shown in Figure 5.10(b).

These participants still appear to exhibit the behavior of this region (focusing on errors of commission), however they were not successful in identifying the true links. We feel this is due to the analysts focusing so much on fixing errors of commission, they cut links without attempting to determine their validity. In 
addition, these participants focus solely on these errors of commission and did not add any additional links to account for errors of omission.

The performance for the participants in this region are showed in Figure 5.6(d) and 5.7(d) for manual and RETRO.net respectively.

High Precision, Low Recall: Cuddeback et al. observed participants from this region tended to improve recall while decreasing the precision as compared to their candidate TMs accuracy. TMs in this region had very few links, as such Cuddeback et al. conjectured that participants spent the majority of their time searching for errors of omission, introducing many true as well as false positive links into their final TM[6]. The performance of participants in this region from the study is shown in Figure 2.3(d).

In the manual and RETRO.net experiments the trend of searching for errors of omission continued. Out of the 8 participants in this region from the RETRO.net and manual studies, all but one improved their recall, often substantially. One major difference seen between Cuddeback's RETRO study and the follow up studies is that $50 \%$ of the participants in this region also increased their precision. In particular this occurred in a region in our manual study were all four analysts greatly improved recall while improving precision by $5 \%$ to $10 \%$. This behavior occurred where precision was between 52 and $67 \%$ and recall was between 21 and 43\%. The behavior of these analysts is shown in Figure 5.10(c).

While this difference is present, we feel the overall trend still holds true that analysts spend their time searching for errors of omission rather than searching the existing candidate links for false positives. These participants were very good at locating correct omitted links and not introduce false positives in the process. When doing this, both the precision and recall increase. 
The results for this region are shown in Figure 5.6(a) for manual and Figure 5.7(a) for RETRO.net.

High Precision, High Recall: Cuddeback et al. observed analysts in this region almost uniformly decrease the accuracy of their candidate TM $(64 \%$ of the participants from this region). When examining the participants who did improve their candidate TM only 1 participant improved substantially. This participant had an initial TM lie on the border of the region ( $80 \%$ precision, $52 \%$ recall) and exhibited similar behavior to participants from the high-precision, low-recall quadrant. For the remainder of those who improved accuracy $\left(f_{2^{-}}\right.$ measure) of their TM, they showed only a $2.5 \%$ increase. The majority of the participants who decreased their overall accuracy did so in a minor way: by less than $8 \%$. Overall, the changes made were rather minor with only $27 \%$ of participants changing their $f_{2}$-measure by more than $10 \%$. The performance for these participants is shown in Figure 2.3(b)[6].

In the manual and RETRO.net experiments, similar observations were made. $72 \%$ and $57 \%$ of participants reduced the accuracy of the TM in the manual and RETRO.net experiments respectively. We also notice similar behavior that as analysts make changes in their TM, these changes tend to be fairly minor. More participants (40\%) made larger changes, greater than $10 \% \Delta f_{2}$. Unfortunately, out of the $40 \%$ of participants who changed their TM accuracy by more than $10 \%$, only one participant improved their accuracy. This participant received a TM on the border (50\% precision, $91 \%$ recall) and showed behavior similar to the low-precision, high-recall region. The analysts' performance from this boundary region is shown in Figure 5.10(d).

The full results for this region are shown in Figure 5.6(b) for manual and 
Figure 5.7(b) for RETRO.net.

\subsubsection{Who improved Accuracy}

Cuddeback et al. observed that 21 out of 33 participants improved their accuracy with the majority of those submitting TMs with an $f_{2}$-measure measure between .6 to $.75(62 \%)$. In addition, a correlation between the initial $f_{2}$-measure and delta $f_{2}$-measure was observed: the lower the initial $f_{2}$-measure the higher the change in $f_{2}$-measure. This is visualized in Figure 5.8(a) and Figure 5.9(a).

Our observations were similar to these findings. One difference seen was that the participants did not as clearly move towards the $f_{2}$-measure between .6 and .75. In contrast, in the manual experiment several participants submitted final TMs lower than .6 (44\%), while in the RETRO.net experiment the majority of participants submitted TMs with an $f_{2}$-measure above $.75(54 \%)$.

Despite these differences there was still a clear correlation between initial $f_{2^{-}}$ measure and change in $f_{2}$-measure. In all three experiments the trend was: the lower the initial $f_{2}$-measure the higher the change in $f_{2}$-measure. See Section 5.5 for a formal analysis on the correlation between initial $f_{2}$-measure and change in $f_{2}$-measure.

In the RETRO.net experiment we saw for the first time an improvement in $f_{2^{-}}$measure from participants receiving high accuracy TMs (over .75 initial $f_{2^{-}}$ measure). The largest improvement from this group was $11 \%$ and an average improvement of $5 \%$. While none of these improvements were large, this trend was new and unique. These participants all received initial TMs with medium precision, 47-76\%, and high recall, 86-91\%. These initial TMs fell where around the boundary between the low-high precision and high recall quadrants. These 


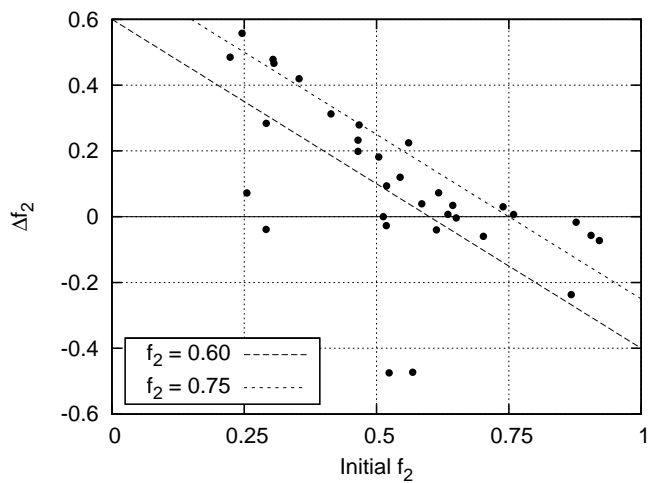

(a) $\Delta f_{2}$ for the RETRO study

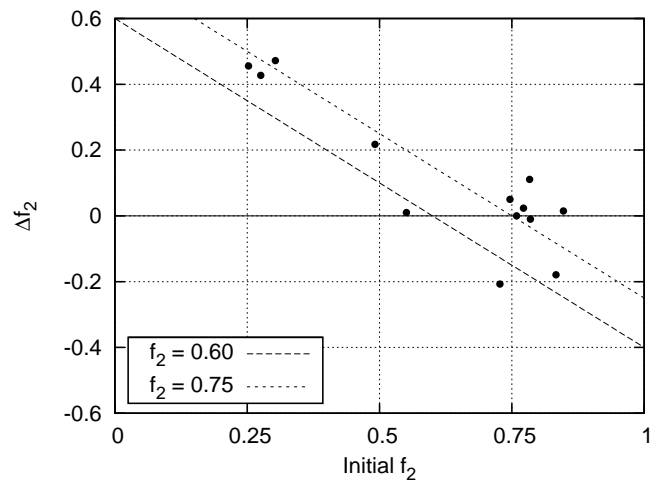

(c) $\Delta f_{2}$ for RETRO.net study

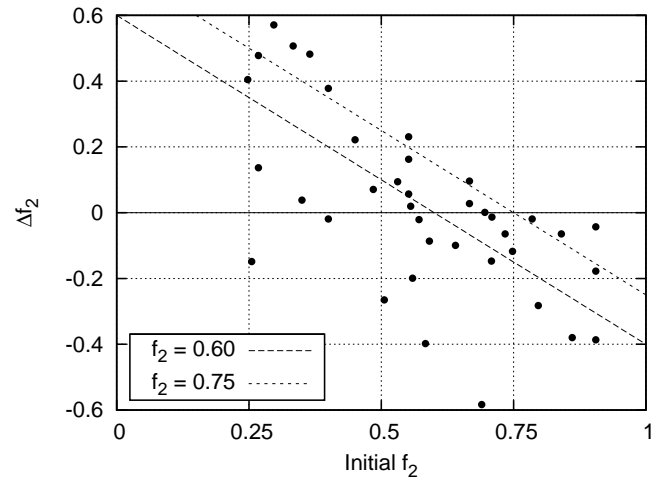

(b) $\Delta f_{2}$ for the manual study

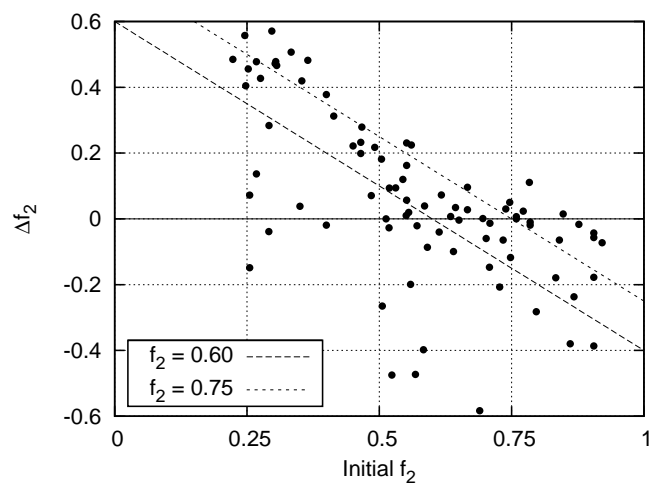

(d) $\Delta f_{2}$ for all studies

Figure 5.8: Change in $f_{2}$-measure for the (a) RETRO, (b) manual, (c) RETRO.net, and (d) all studies combined.

participants recognized they had the majority of correct links and focused on errors of commission. This is the same behavior we noticed in the low precision, high recall region. The difference in behavior was these analysts recognized true links with high accuracy and only removed false positives.

These findings are visualized in Figures 5.8(b) and 5.9(b) for the manual experiment and Figures 5.8(c) and 5.9(c) for RETRO.net. In addition we show Figures 5.8(d) and 5.9(d) with all three data combined to show the overall trends. 


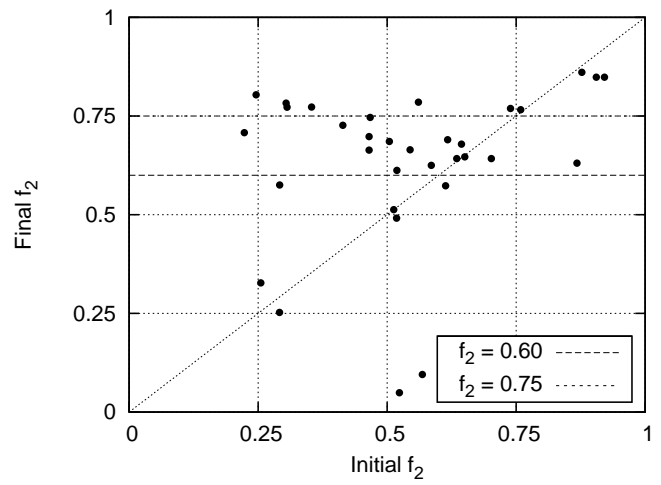

(a) Final $f_{2}$-measure for RETRO study

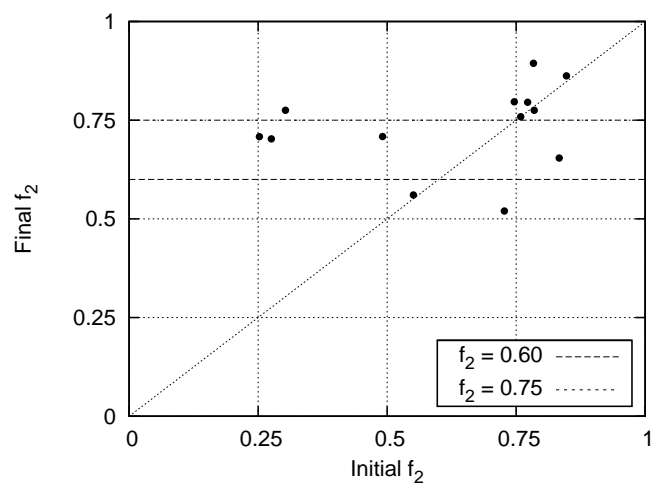

(c) Final $f_{2}$-measure for RETRO.net study

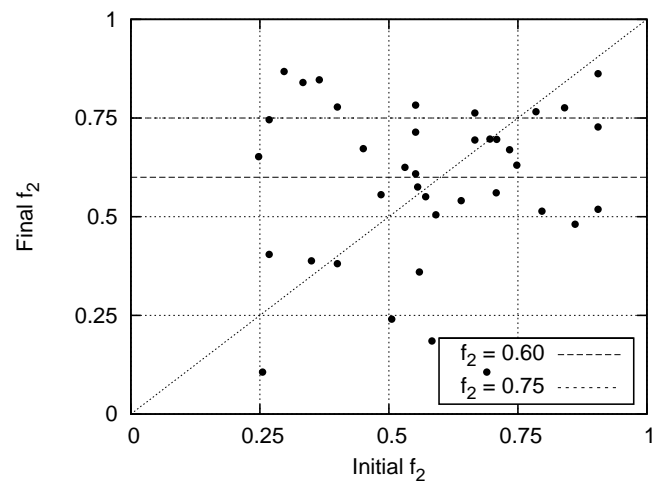

(b) Final $f_{2}$-measure for manual study

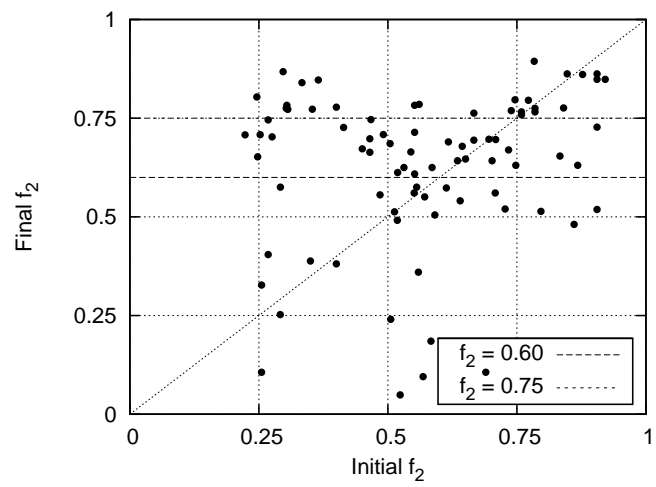

(d) Final $f_{2}$-measure for all studies

Figure 5.9: Inital $f_{2}$-measure vs. Final $f_{2}$-measure for the (a) RETRO, (b) manual, (c) RETRO.net, and (d) all studies combined.

\subsubsection{Outliers}

In our experiments we had some participants who we view as outliers. The performance of these participants is shown in Figure 5.10(a). Of these outliers, two are marked as outliers as they greatly decreased their TM accuracy and turned in candidate TMs with a very low number of links. Judging by the analysts surrounding them we believe that this behavior is not representative of the region and is caused by analysts who did not understand the task at all.

The remaining two outliers are participants who did not vet the candidate TM, instead they turned in an exact copy of the TM presented to them. While 


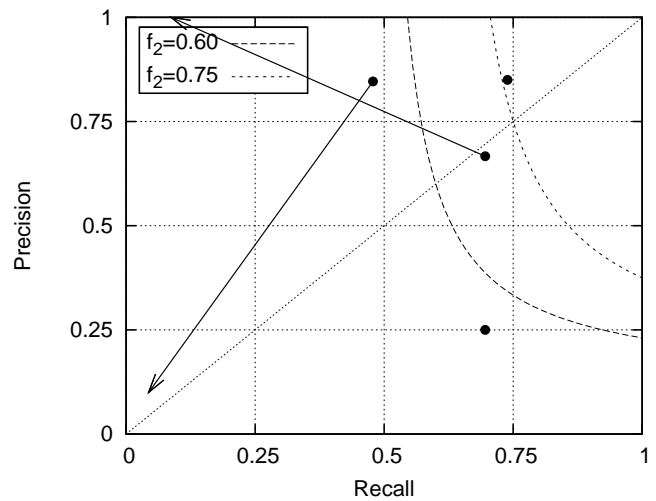

(a) Outliers from all three studies

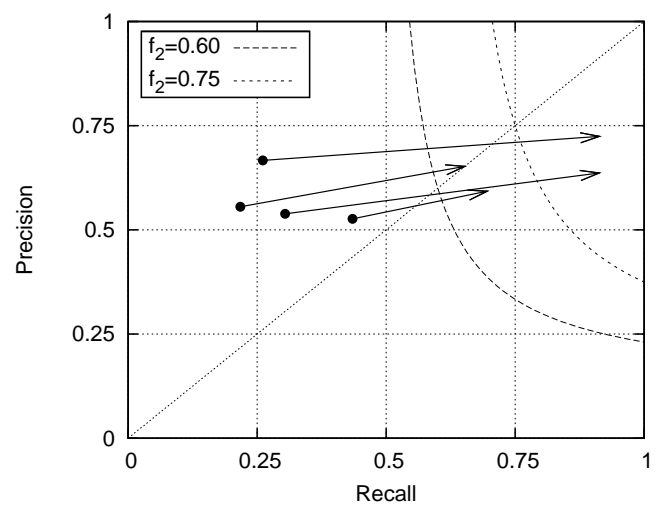

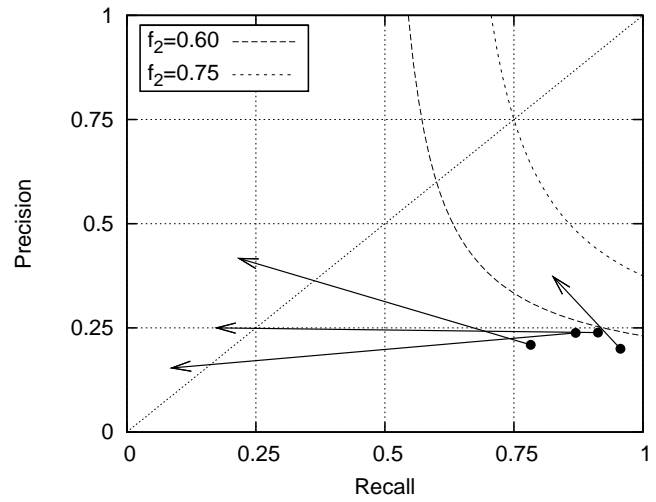

(b) Performance when analysts vet largest TMs

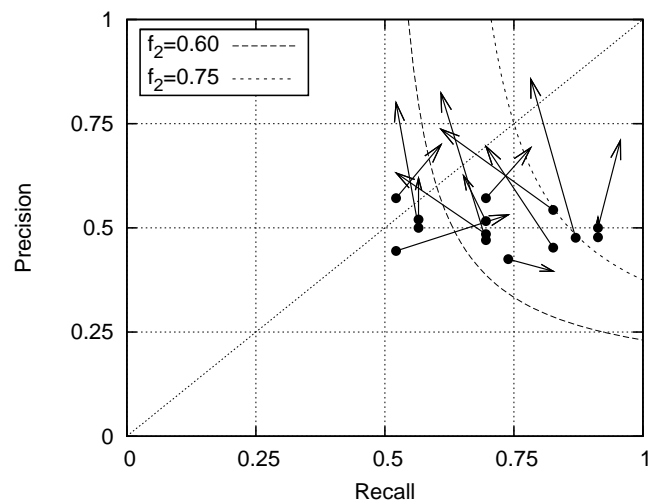

(c) Good performance from analysts in high (d) Analyst performance from the boarder reprecision, low recall cluster

gion between low and high precision and high recall

Figure 5.10: Analyst performance for (a) outliers, (b) analysts vetting the largest TMs, (c) Positive region in high precision, low recall area, and (d) border region between low and high precision with high recall.

one of the analysts did start with a decent TM, $85 \%$ precision and $74 \%$ recall, we feel it unlikely that an analyst performing validation would not change a single link in the TM.

There was a third group of potential outliers from analysts receiving the largest TMs. This group was discussed in the paragraph talking about low precision high recall trends. As mentioned there, we do not currently classify these analysts as outliers since several followed similar patterns, however these analysts are still suspect due to their overall performance. Additional data points need to 
Table 5.1: Influence of initial precision and initial recall on response variables (degrees of freedom: 2,81 )

\begin{tabular}{|l|l|l|l|}
\hline Response Variable & $R_{a d j}^{2}$ & F-value & Sig. (pval) \\
\hline FinPrec & 0.117 & 6.484 & $\mathbf{0 . 0 0 2}$ \\
\hline FinRec & 0.010 & 0.416 & 0.661 \\
\hline FinF2 & 0.014 & 0.592 & 0.556 \\
\hline$\Delta$ Prec & 0.481 & 37.482 & $\mathbf{0 . 0 0 0 1}$ \\
\hline$\Delta$ Rec & 0.485 & 38.140 & $\mathbf{0 . 0 0 0 1}$ \\
\hline$\Delta$ F2 & 0.464 & 35.054 & $\mathbf{0 . 0 0 0 1}$ \\
\hline
\end{tabular}

be collected to formally determine if this is indeed representative behavior from this region or outliers.

\subsection{Statistical Analysis}

The informal observations are useful in providing insight into semi-automated tracing. However, we feel it is important to expand by conducting formal analysis to determine which factors influence accuracy of the final TM. In particular we examined the variables, baseline and observed, to see which ones, by themselves or in combination provided a statistically significant impact on the final TM accuracy.

\subsubsection{Baseline independent variables}

Table 5.1 shows the influence of the pair of independent variables, Initial Precision and Initial Recall, on the dependant variables. To preform this analysis we conducted a multi-variable regression analysis. We report the adjusted Rsquare value $\left(R_{a d j}^{2}\right)$, the F-value, and the significance level. For our analysis we use a significance level of 0.05 . 
Table 5.2: Influence of initial $f_{2}$-measure on response variables (degrees of freedom: 1, 82)

\begin{tabular}{|l|l|l|l|}
\hline Response Variable & $R_{a d j}^{2}$ & F-value & Sig. (pval) \\
\hline FinPrec & 0.044 & 4.836 & $\mathbf{0 . 0 3 1}$ \\
\hline FinRec & 0.005 & 1.403 & 0.240 \\
\hline FinF2 & 0.021 & 2.816 & 0.097 \\
\hline$\Delta$ Prec & 0.033 & 3.823 & 0.054 \\
\hline$\Delta$ Rec & 0.347 & 45.130 & $\mathbf{0 . 0 0 0 1}$ \\
\hline$\Delta$ F2 & 0.422 & 61.693 & $\mathbf{0 . 0 0 0 1}$ \\
\hline
\end{tabular}

As can be seen from the table, the initial accuracy of the TM has statistical significance on the final TM precision, as well as on delta precision, delta recall, and delta $f_{2}$-measure. This statistical analysis shows that given the initial precision-recall pair we should be able to determine with statistical significance what the final precision will be as well as the change in all three accuracy variables.

Using initial $f_{2}$-measure as a one-dimensional surrogate for the initial TM accuracy, we conducted a linear regression analysis on its impact to the dependant variables. The results are summarized in Table 5.2. As can be seen from the table initial $f_{2}$-measure statistically significantly influences final precision, delta recall, and delta $f_{2}$-measure. As shown here, the $f_{2}$-measure can act as a surrogate, however as it is a more coarse measure, it does not influence delta precision.

Finally, we went with an even coarser measure and examined the statistical impact on the initial TM quadrant on the accuracy of the final TM. The quadrants were described in Section 5.4.2. We conducted a one-way analysis of variance (ANOVA) to determine the impact on the dependent variables. Table 5.3 shows the results of this analysis. In this analysis QI is the low-precision, low-recall quadrant, QII is the low-precision, high-recall quadrant, QIII is the 
Table 5.3: Influence of Starting Quadrant on Response Variables (degrees of freedom: 3,80 )

\begin{tabular}{|l|l|l|l|}
\hline Response Variable & $R_{a d j}^{2}$ & F-value & Sig. (pval) \\
\hline FinPrec & 0.132 & 5.206 & $\mathbf{0 . 0 0 2}$ \\
\hline FinRec & 0.010 & 0.726 & 0.539 \\
\hline Fin $f_{2}$ & 0.011 & 1.320 & 0.274 \\
\hline$\Delta$ Prec & 0.403 & 19.666 & $\mathbf{0 . 0 0 0 1}$ \\
\hline$\Delta \operatorname{Rec}$ & 0.361 & 16.645 & $\mathbf{0 . 0 0 0 1}$ \\
\hline$\Delta f_{2}$ & 0.354 & 16.184 & $\mathbf{0 . 0 0 0 1}$ \\
\hline
\end{tabular}

high-precision, low-recall quadrant, and QIV is the high-precision, high-recall quadrant.

As can be seen by the Table 5.3 the starting quadrant has statistically significant impact on final precision and all three deltas. This once again confirms our findings that are informally presented in Section 5.4.2, that the quadrant the participant begins in has statistically significant impact on what the analyst will produce as a final TM.

\subsubsection{Observed Independent Variables}

To examine the influence of the Observed Independent Variables we conducted statistical analysis on each variable, controlling for the baseline independent variables to prevent influence of precision-recall distribution to be a factor. This analysis is shown in Table 5.4.

Of the eleven observed independent variables, only time to complete the tracing task(Time) is continuous, the remaining variables were categorical. For Time 
Table 5.4: Analysis for observed independent variables controlling for initial TM precision and recall

\begin{tabular}{|c|c|c|c|c|c|c|c|c|c|c|c|c|}
\hline \multicolumn{2}{|l|}{ Response } & Location & Procedure & $\overline{\text { SEExp }}$ & 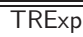 & Time & Grade & TrConf & Opinion & MissingEff & ValidEff & Prepared \\
\hline \multirow{3}{*}{$\begin{array}{l}\text { FinPrec } \\
R_{a d j}^{2}=0.122\end{array}$} & 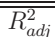 & 0.124 & 0.105 & 0.144 & 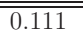 & 0.122 & 0.144 & $\overline{c 0.079}$ & 0.127 & 0.052 & 0.117 & 0.096 \\
\hline & $F$ & 1.661 & 0.476 & 0.723 & 1.477 & 1.028 & 1.649 & 0.067 & 1.265 & 0.308 & 1.142 & 0.989 \\
\hline & $p$ & 0.314 & 0.623 & 0.489 & 0.228 & 0.363 & 0.171 & 0.796 & 0.288 & 0.907 & 0.348 & 0.431 \\
\hline \multirow{3}{*}{$\begin{array}{l}\text { FinRec } \\
R_{a d j}^{2}=0.02\end{array}$} & $R_{a d j}^{2}$ & 0.014 & 0.035 & 0.025 & 0.022 & 0.025 & 0.015 & 0.028 & 0.040 & 0.037 & 0.146 & 0.047 \\
\hline & $F$ & 1.057 & 3.065 & 0.482 & 0.143 & 0.114 & 1.605 & 0.0001 & 0.025 & 0.678 & 3.562 & 0.548 \\
\hline & $p$ & 0.307 & 0.052 & 0.619 & 0.707 & 0.736 & 0.182 & 0.992 & 0.975 & 0.641 & 0.007 & 0.739 \\
\hline \multirow{3}{*}{$\begin{array}{l}\text { FinF2 } \\
R_{a d j}^{2}=0.014\end{array}$} & $R_{a d j}^{2}$ & 0.018 & 0.027 & 0.016 & 0.020 & 0.020 & 0.004 & 0.030 & 0.031 & 0.038 & 0.116 & 0.044 \\
\hline & $F$ & 0.396 & 2.546 & 0.781 & 0.227 & 0.021 & 1.289 & 0.117 & 0.120 & 0.684 & 2.974 & 0.593 \\
\hline & $p$ & 0.531 & 0.085 & 0.462 & 0.635 & 0.885 & 0.282 & 0.773 & 0.887 & 0.637 & 0.018 & 0.705 \\
\hline \multirow{3}{*}{$\begin{array}{l}\Delta \text { Prec } \\
R_{a d j}^{2}=0.456\end{array}$} & $R_{a d j}^{2}$ & 0.472 & 0.461 & 0.478 & 0.484 & 0.475 & 0.484 & 0.475 & 0.478 & 0.447 & 0.483 & 0.473 \\
\hline & $F$ & 1.661 & 0.476 & 0.723 & 1.477 & 1.028 & 1.649 & 0.067 & 1.265 & 0.308 & 1.142 & 0.989 \\
\hline & $p$ & 0.201 & 0.623 & 0.489 & 0.228 & 0.314 & 0.171 & 0.796 & 0.288 & 0.907 & 0.348 & 0.431 \\
\hline \multirow{3}{*}{$\begin{array}{l}\Delta \operatorname{Rec} \\
R_{a d j}^{2}=0.446\end{array}$} & $R_{a d j}^{2}$ & 0.473 & 0.498 & 0.465 & 0.467 & 0.444 & 0.488 & 0.476 & 0.436 & 0.468 & 0.541 & 0.463 \\
\hline & $F$ & 1.057 & 3.065 & 0.482 & 0.143 & 0.114 & 1.605 & 0.0001 & 0.025 & 0.678 & 3.562 & 0.548 \\
\hline & $p$ & 0.307 & 0.052 & 0.619 & 0.707 & 0.736 & 0.182 & 0.992 & 0.975 & 0.641 & 0.007 & 0.739 \\
\hline \multirow{3}{*}{$\begin{array}{l}\Delta \mathrm{F} 2 \\
R_{a d j}^{2}=0.417\end{array}$} & $R_{a d j}^{2}$ & 0.450 & 0.480 & 0.461 & 0.450 & 0.412 & 0.457 & 0.459 & 0.404 & 0.438 & 0.511 & 0.442 \\
\hline & $F$ & 0.918 & 3.286 & 1.314 & 0.116 & 0.065 & 1.226 & 0.298 & 0.006 & 0.544 & 3.490 & 0.666 \\
\hline & $p$ & 0.341 & 0.043 & 0.275 & 0.734 & 0.799 & 0.307 & 0.587 & 0.994 & 0.742 & 0.007 & 0.650 \\
\hline
\end{tabular}

we used multiple linear regression analysis while for the others we utilized analysis of covariance (ANCOVA) analysis.

When observing the variables and their impact on the dependant variables, only one, ValidEff, has statistically significant impact. This variable measured the participant's self-reported amount of time they spent vetting the candidate links provided to them (as apposed to searching for links missed by the candidate TM). The participants were given a six point scale from 0-5 where 0 meant "never preformed this activity" and 5 meant "performed this activity for every single link". When we examined why this had a statistical impact we found that the majority of participants reported a 0-3 (74\%) while 17\% of participants answered a 4 or $5^{1}$.

When examining the performance of analysts who reported a large validation effort ( 4 or 5 on the survey) we notice that they produced final TMs with $20.5 \%$ lower recall than the average of those who reported 0-3. In addition this group was the only whose mean recall was negative, and by a substantial amount. The

\footnotetext{
${ }^{1}$ the remaining participants did not answer this question
} 
Table 5.5: Influence of ValidEff on response variables

\begin{tabular}{|ll||l|l|}
\hline Response & & $0-3$ & $4-5$ \\
\hline & $N$ & 62 & 14 \\
\hline FinRec & $\bar{x}$ & 65.25 & $\mathbf{4 4 . 7 2}$ \\
& $s$ & 19.98 & 23.28 \\
\hline Fin $f_{2}$ & $\bar{x}$ & 62.36 & $\mathbf{4 5 . 6 6}$ \\
& $s$ & 17.98 & 22.25 \\
\hline$\Delta \operatorname{Rec}$ & $\bar{x}$ & 5.18 & $\mathbf{- 2 4 . 2 2}$ \\
& $s$ & 26.71 & 30.73 \\
\hline$\Delta f_{2}$ & $\bar{x}$ & 7.06 & $\mathbf{- 1 4 . 5 5}$ \\
& $s$ & 22.33 & 23.59 \\
\hline
\end{tabular}

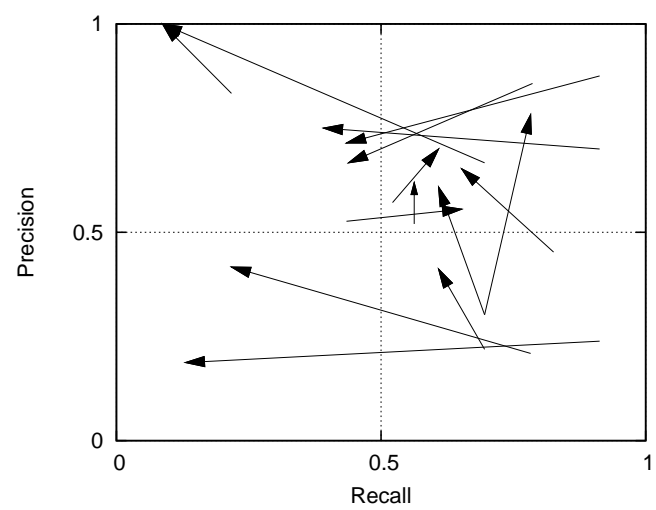

Figure 5.11: Participants with ValidEff values of 4 or 5

average recall for this group decreased by $24.2 \%$. This influence is shown in Table 5.5. In addition, Figure 5.5.2 shows the performance of the participants who answered a 4 or 5 . As can be seen in this figure, most participants had initial TMs in high recall and all but one lead to a decrease (often substantial decrease) in recall. This self-reported behavior is consistent with the behavior reported by Cuddeback and informally confirmed above in Section 5.4.2. Participants given high recall spend the majority of their time vetting links. Due to errors of analyst judgement during link validation, valid links were removed resulting in negative delta recall. 


\subsection{Summary of Key Findings}

Our informal and formal analysis highlighted several key takeaways from this study. First, we found that the best indicator for analyst performance was the initial TM accuracy. In fact, only one other independent variable we collected, ValidEff, showed any statistically significant impact on the accuracy of the final TM. However, as shown in our analysis this was actually a negative correlation. Analysts who reported spending more time validating links decreased their overall TM accuracy.

The second key observation is that analysts have a very difficult time determining what the true TM is. None of the 84 participants recovered the true TM. In fact, only $9.5 \%$ on analysts ( 8 out of 84 ) returned TMs with precision and recall greater than $75 \%$. This is true despite 8 analysts having initial TMs in this region ( $50 \%$ stayed in this region).

A third key finding was that analysts seem to have an idea of the size of the true TM. When analysts receive large TMs they do predictable actions, they focus mainly on link removal, improving precision often at the detriment of recall. Analysts receiving small TMs tend to do the opposite, focus solely on searching for missing links, improving recall at the cost of precision. When analysts receive high accuracy TMs they normally decrease the overall accuracy of the TM. Finally when analysts receive low accuracy TMs the analysts seem to recognize a high occurrence of false positives and removing them. After removal of the false positives the analysts recognize the TM is to small and search for missing links.

The final key finding was the accuracy of the initial automatically-generated TM appears to be a bad method in measuring the quality of a semi-automated tracing process. When analyzing the variables of our studies we find that this 
accuracy is the best predictor we have observed, however in the opposite direction. In fact, we see the analysts who produce some of the highest quality TMs were analysts receiving the lowest quality TMs. 


\section{Chapter 6}

\section{Naturally Generated TM Experiment Design}

Our second experimental framework allows us to test how analysts interact with real IR and filtering methods while vetting candidate TMs. Specifically in our experimental design we shift from utilizing artificially generated TMs, as used in Section 4, to naturally generated TMs. Here by "naturally generated TMs" we mean TMs generated automatically utilizing real IR and filtering methods. By utilizing these naturally generated TMs we shift from directly controlling precision and recall of the candidate TM. Instead we control the methods which generate TMs.

Additionally we add control over the user interface used to display the candidate links as well as user feedback processing. While our pilot study does not utilize these additional pieces they are included into our full experimental design. This study is detailed in Section 6.2. 


\subsection{Software tools used}

We created a framework to control the IR method, filtering technique, user feedback, and user interface. We modified RETRO.net to utilize this framework by connecting the user log-in to control each of the framework parameters and utilize these parameters when generating and displaying the candidate TM. These modifications established a plugin architecture where researchers can design new IR methods, filtering techniques, user feedback, or UI and easily install them into the tool to be utilized during experiments. In addition, we implemented several filtering techniques outlined in Section 6.1.1.

\subsubsection{Filtering techniques}

Running IR methods typically generates a list of candidate links with relevance scores. These candidate links can be viewed as-is without filtering any links out. However, it may be advantageous to filter out candidate links in various ways in order to reduce the number of links a human must analyze. A few of these options are described below.

Value Filtering. One of the simplest ways to filter links is by setting a threshold value and removing all links with relevance scores below this threshold. Since relevance scores range in value from zero to one, the allowable threshold values are in this range. This filtering is applied on all potential links equally.

Global Filtering. Candidate links can be filtered on a global scale. To accomplish this all candidate links are sorted by relevance score and than the top number of candidate links are kept and the rest are removed (filtered out). An 
additional, but similar method, can filter out candidate links that are not in the top X percent of the links. Both of these methods are referred to as global filtering. As an example, if the IR method returns 200 candidate links, a global filter can be applied to return the top 20 links or the top 15\% (top 35 links).

Local Filtering. Candidate links can be filtered on a per high-level element basis. To accomplish this, all candidate links are separated by their high-level element into different lists sorted by relevance score. In this manner if we are tracing from requirement to test cases we can show the analyst the top ' $\mathrm{X}$ ' test cases per requirement or top 'X' percent of test cases per requirement.

These filters can also be combined together. One example of this is applying a global filter, than a local filter on those results.

\subsubsection{User Interfaces}

Three different user interfaces have been designed for the full experiment. Due to time limitations currently only one has been developed. However, to fully explain our experimental design we give an overview of all three user interactions.

Free Form Browsing. This interface was the interface described in Section 4.1.2 and shown in Figures 4.3(a), 4.3(b), and 4.4. This interface provides three unique interaction types. The first, recommended tab (Figure 4.3(a)), shows the recommended links and allows the user to confirm or reject these links. The second, keyword tab (Figure 4.4), allows the user to search for links by specific keywords. This tab may be utilized to search for additional links or confirm recommended links. Finally the third tab, "all" tab (Figure 4.3(b)), displays the 
user all potential links allowing the user to search for any missing links.

All three interaction types are free-form, allowing the user to switch between tasks, i.e. confirming links or searching for additional links, as well as freely switch between any two high and low level document elements at any time. The free-form browsing UI does not control the activities of the analyst.

Local Browsing. This interface is designed to force the user to consider links one-ny-one in a predefined order. The order is based on splitting the links by the high-level element and showing all candidate links for one high-level element prior to advancing onto the next. This interface would display each recommended link for a particular high-level element one at a time and have the analyst render a decision on the link. After the user vets the candidate links, or when they choose to complete the recommended link process they would be allowed to search for missing links for this high level element similarly to the "all" tab from the free browsing UI. However, this interface will not allow jumping from one high-level element to another as was possible in the free form browsing interface.

Global Browsing. This interface will sort all candidate links by relevance score and show the user one candidate link at a time in descending order without regard for the high-level element of this link. After the user vets all candidate links or when the user chooses to complete the recommended link process they would be allowed to search for any missing links. This search process would be very similar to the "all" tab from the free browsing UI. 
Table 6.1: ChangeStyle Pilot Study Experimental Design

\begin{tabular}{|l|c|c|c|c|r|}
\hline Method Used & Filter Used & Filter Value & Number Participants & Initial Precision & Initial Recall \\
\hline TF-IDF & None & NA & 2 & $4.9 \%$ & $100 \%$ \\
\hline OKAPI & local & 1 & 2 & $46.9 \%$ & $62.5 \%$ \\
\hline TF-IDF & local & 1 & 3 & $50.0 \%$ & $66.7 \%$ \\
\hline TF-IDF & local & 3 & 3 & $18.8 \%$ & $75.0 \%$ \\
\hline TF-IDF & value & 0.25 & 4 & $73.3 \%$ & $45.8 \%$ \\
\hline OKAPI & value & 0.25 & 3 & $70.6 \%$ & $50.0 \%$ \\
\hline TF-IDF & global & 96 & 3 & $18.8 \%$ & $75.0 \%$ \\
\hline OKAPI & global & 96 & 3 & $19.8 \%$ & $79.2 \%$ \\
\hline OKAPI & global & 23 & 2 & $52.2 \%$ & $50.0 \%$ \\
\hline
\end{tabular}

\subsection{Pilot Study Experimental design}

A pilot study was conducted in order to examine how the participants interact with candidate TMs generated using real IR methods and filtering techniques. Our framework allowed us many possible combinations to examine ${ }^{1}$. We decided to concentrate on just a few due to the sample size of our experiment. We decided to focus on two IR methods (TF-IDF and Okapi) and four filter techniques (none, local top 'X', global top 'X', and value). In addition to these combinations we also explored using different values for these filter types. When assigning a combination to a participant we used the same round-robin process as the RETRO.net experiment. To obtain some overlap and attempt to isolate outliers each of these combinations were assigned to multiple individuals. In the experiment described in Section 4 this replication was done by assigning TMs with similar (but not the same) accuracy. In our experiment this was not possible as the control variables were not continuous. Our pilot study experimental design is shown in Table 6.2 for ChangeStyle and Table 6.2 for HIPAA. Additionally we visually display the initial TM accuracy in Figure 6.1(a) for ChangeStyle, Figure 6.1(b) for HIPAA, and $6.1(\mathrm{c})$ for both datasets.

\footnotetext{
${ }^{1} 108$ total combinations: 3 IR methods, 6 filter types, 3 different user interfaces, and use of user-feedback
} 
Table 6.2: HIPAA Pilot Study Experimental Design

\begin{tabular}{|l|c|c|c|c|r|}
\hline Method Used & Filter Used & Filter Value & Number Participants & Initial Precision & Initial Recall \\
\hline TF-IDF & None & NA & 2 & $6.8 \%$ & $81.3 \%$ \\
\hline OKAPI & local & 1 & 2 & $40.0 \%$ & $25.0 \%$ \\
\hline TF-IDF & local & 3 & 2 & $26.7 \%$ & $50.0 \%$ \\
\hline OKAPI & value & 0.25 & 2 & $57.1 \%$ & $25.0 \%$ \\
\hline
\end{tabular}

\subsection{Dataset}

We used two different datasets in this experiment. The first one was ChangeStyle described in Section 4.3.2. We also wanted to address the "single dataset" threat to validity of the first experiment. For this we utilized a second dataset, HIPAAClearHealth.

\subsubsection{HIPAA-ClearHealth Dataset}

The Health Insurance Portability and Accountability Act (HIPAA)[1] is enacted by the United States of America Congress to protect health insurance coverage. In addition, it focuses on addressing the privacy and security of health care-related electronic data. We focus only on the privacy and security regulations. These regulations are shown in Appendix I.

This dataset focuses on tracing from the HIPAA electronic regulations to a software requirements specification (SRS) of healthcare products. In the full dataset HIPAA electronic protection regulations are traced to requirements for 10 different health care software systems[4]. We chose one of these systems, ClearHeath, to trace.

The HIPAA-ClearHealth dataset includes 44 requirements, 11 HIPAA regulations, and 16 true-validated links. It focuses on an important goal of traceability, software satisfaction. In other words, the goal of the tracing task is to establish 

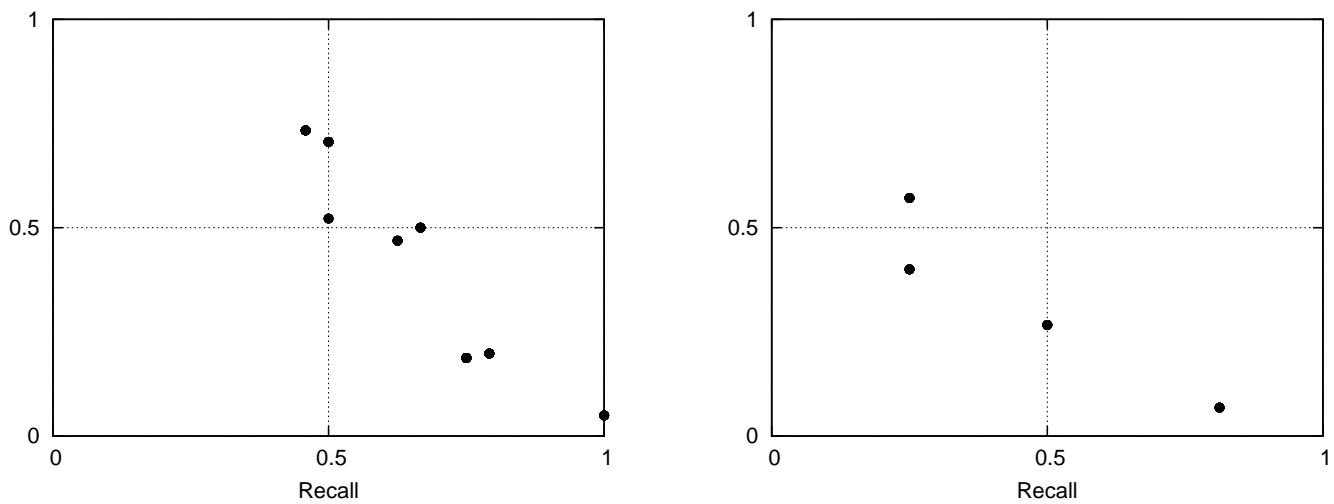

(a) Starting TM accuracy for ChangeStyle (b) Starting TM accuracy for HIPAA dataset dataset

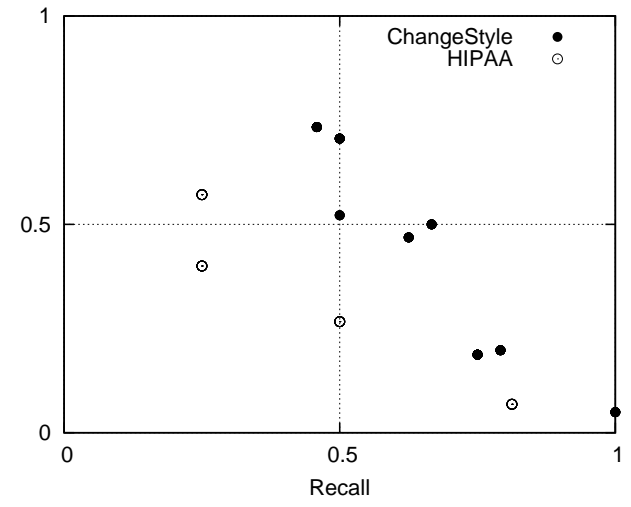

(c) Starting TM accuracy comparing ChangeStyle and HIPAA datasets

Figure 6.1: Initial TM accuracy of (a) ChangeStyle, (b) HIPAA, and (c) both datasets

whether the software system meets the regulatory requirements of the HIPAA federal regulations.

\subsection{Information collected}

The pilot experiment expands upon the previous experiment. We continued to collect the meta-information from surveys described in Section 4.4. However, we modified the Baseline independent variables. Rather than controlling 
Table 6.3: Baseline Independent Variables

\begin{tabular}{|l|c|c|r|}
\hline Variable & Abbreviation & Scale & Used in Pilot \\
\hline IR method & IRMethod & Okapi,TF-IDF,LTU & Yes $^{2}$ \\
\hline Filter Technique & FilterType & None,Global,Local,Value & Yes \\
\hline Filter Value & FilterVal & Local \& Global: [0,maxLinks], Value: $[0,1]$ & Yes \\
\hline User Interface & UI & FreeBrowse, Local, Global & No \\
\hline Feedback Processing & Feedback & Yes,No & No \\
Initial Precision & SPrec & {$[0,1]$} & Indirect \\
\hline Initial Recall & SRec & {$[0,1]$} & Indirect \\
\hline Initial F2 & SF2 & {$[0,1]$} & Indirect \\
\hline Initial Quadrant & SQuadrant & $\{Q 1, Q 2, Q 3, Q 4\}$ & Indirect \\
\hline
\end{tabular}

the precision and recall values we now control what IR method to use, filtering technique, and filtering value. In addition, our experimental framework provides the capability to control additional baseline independent variables, however these were not used in our pilot study due to our user base. These variables include user feedback processing and user interface(UI).

It should also be noted that while we no longer are directly controlling precision and recall we still are able to track these variables as well as indirectly control them through the other variables chosen. Due to this we keep these as baseline independent variables. The new set of baseline independent variables is shown in Table 6.3.

This change is a step towards our ultimate goal of being able to create a semi-automated requirements tracing tool that academia and industry can use. By changing from artificially generated TMs to naturally generated, we were able to investigate how humans interact with these naturally generated TMs and, more importantly, begin to understand what parameters to use to lead to the best human $\rightarrow$ computer interaction such that the final TMs are consistently of high accuracy. 


\subsection{Research questions}

We look to examine how human analysts transform traceability information from candidate TMs in final TMs. In particular we break this question down to target semi-automated requirements tracing using real IR and filtering methods. Specifically we ask the following questions:

- How does the IR method used in creating a candidate TM impact the final TM?

- How does the filtering method used on the candidate TM impact the final TM? In particular:

- Are analysts more successful when global, local, or value filters have been applied?

- Is analyst performance consistent cross multi datasets?

- Is there a difference between analyst performance when using naturallygenerated TMs vs. artificially generated TMs? 


\section{Chapter 7}

\section{Naturally Generated TM Experiment Results}

We conducted our pilot study in three classes at two locations, Cal Poly and University of Kentucky, using two different datasets, ChangeStyle and HIPAA. In total, we collected data from 31 participants, 23 using ChangeStyle and 8 using HIPAA. Table 7.1 gives an overview of our experiments.

\subsection{ChangeStyle}

For our ChangeStyle experiment using naturally-generated TMs we used 9 different combinations of IR method, filtering, and filter value. Table 6.2 shows

\begin{tabular}{|l|l|l|}
\hline Location & Dataset used & number of participants \\
\hline University 1 & ChangeStyle & 5 \\
\hline University 2 & ChangeStyle & 18 \\
\hline University 2 & HIPAA & 8 \\
\hline
\end{tabular}

Table 7.1: Overview of our natural TM generation experiments 
Table 7.2: Performance for analysts using naturally generated TMs vetting the ChangeStyle dataset

\begin{tabular}{|c|c|c|c|c|c|c|c|c|c|}
\hline $\begin{array}{l}\text { UserID } \\
\text { UserA }\end{array}$ & $\begin{array}{l}\text { IR Method } \\
\text { TF-IDF }\end{array}$ & $\begin{array}{l}\text { Filter Type } \\
\text { None }\end{array}$ & $\begin{array}{l}\text { Filter Value } \\
\text { NA }\end{array}$ & $\begin{array}{l}\text { Initial Precision } \\
4.9 \%\end{array}$ & $\begin{array}{l}\text { Initial Recall } \\
100 \%\end{array}$ & $\begin{array}{l}\text { Initial } f_{2} \\
20.6 \%\end{array}$ & $\begin{array}{l}\text { Final Precision } \\
37.5 \%\end{array}$ & $\begin{array}{l}\text { Final Recall } \\
75.0 \%\end{array}$ & $\begin{array}{l}\text { Final } f_{2} \\
62.5 \%\end{array}$ \\
\hline UserB & TF-IDF & None & $\mathrm{NA}$ & $4.9 \%$ & $100 \%$ & $20.6 \%$ & $73.1 \%$ & $79.2 \%$ & $77.9 \%$ \\
\hline UserC & Okapi & Value & 0.25 & $73.3 \%$ & $45.8 \%$ & $49.6 \%$ & $28.4 \%$ & $95.8 \%$ & $65.0 \%$ \\
\hline UserD & Okapi & $\begin{array}{l}\text { Value } \\
\end{array}$ & 0.25 & $73.3 \%$ & $45.8 \%$ & $49.6 \%$ & $78.6 \%$ & $91.7 \%$ & $88.7 \%$ \\
\hline UserE & Okapi & Value & 0.25 & $73.3 \%$ & $45.8 \%$ & $49.6 \%$ & $41.0 \%$ & $66.7 \%$ & $59.3 \%$ \\
\hline$\overline{\text { UserF }}$ & Okapi & Value & 0.25 & $73.3 \%$ & $45.8 \%$ & $49.6 \%$ & $63.2 \%$ & $50.0 \%$ & $52.2 \%$ \\
\hline UserG & TF-IDF & Value & 0.25 & $70.6 \%$ & $50.0 \%$ & $53.1 \%$ & $55.2 \%$ & $66.7 \%$ & $64.0 \%$ \\
\hline UserH & TF-IDF & Value & 0.25 & $70.6 \%$ & $50.0 \%$ & $53.1 \%$ & $52.9 \%$ & $37.5 \%$ & $39.8 \%$ \\
\hline UserI & TF-IDF & Value & 0.25 & $70.6 \%$ & $50.0 \%$ & $53.1 \%$ & $81.0 \%$ & $70.8 \%$ & $72.7 \%$ \\
\hline UserJ & Okapi & Local & 1 & $46.9 \%$ & $62.5 \%$ & $58.6 \%$ & $78.3 \%$ & $75.0 \%$ & $75.6 \%$ \\
\hline UserK & Okapi & Local & 1 & $46.9 \%$ & $62.5 \%$ & $58.6 \%$ & $75.0 \%$ & $75.0 \%$ & $75.0 \%$ \\
\hline UserL & TF-IDF & Local & 1 & $50.0 \%$ & $66.7 \%$ & $62.5 \%$ & $73.1 \%$ & $79.2 \%$ & $77.9 \%$ \\
\hline UserM & TF-IDF & Local & 1 & $50.0 \%$ & $66.7 \%$ & $62.5 \%$ & $92.9 \%$ & $54.2 \%$ & $59.1 \%$ \\
\hline UserN & TF-IDF & Local & 3 & $18.8 \%$ & $75.0 \%$ & $46.9 \%$ & $71.4 \%$ & $83.3 \%$ & $80.7 \%$ \\
\hline UserO & TF-IDF & Local & 3 & $18.8 \%$ & $75.0 \%$ & $46.9 \%$ & $65.5 \%$ & $79.2 \%$ & $76.0 \%$ \\
\hline UserP & TF-IDF & Local & 3 & $18.8 \%$ & $75.0 \%$ & $46.9 \%$ & $38.0 \%$ & $79.2 \%$ & $65.1 \%$ \\
\hline UserQ & TF-IDF & Global & 96 & $18.8 \%$ & $75.0 \%$ & $46.9 \%$ & $64.0 \%$ & $66.7 \%$ & $66.1 \%$ \\
\hline UserR & TF-IDF & Global & 96 & $18.8 \%$ & $75.0 \%$ & $46.9 \%$ & $57.6 \%$ & $79.2 \%$ & $73.6 \%$ \\
\hline UserS & TF-IDF & Global & 96 & $18.8 \%$ & $75.0 \%$ & $46.9 \%$ & $56.7 \%$ & $70.8 \%$ & $67.5 \%$ \\
\hline UserT & Okapi & Global & 96 & $19.8 \%$ & $79.2 \%$ & $49.5 \%$ & $58.1 \%$ & $75.0 \%$ & $70.9 \%$ \\
\hline UserU & Okapi & Global & 96 & $19.8 \%$ & $79.2 \%$ & $49.5 \%$ & $51.4 \%$ & $75.0 \%$ & $68.7 \%$ \\
\hline UserV & Okapi & Global & 96 & $19.8 \%$ & $79.2 \%$ & $49.5 \%$ & $79.2 \%$ & $79.2 \%$ & $79.2 \%$ \\
\hline UserW & Okapi & Global & 23 & $52.5 \%$ & $50.0 \%$ & $50.4 \%$ & $58.6 \%$ & $70.8 \%$ & $68.0 \%$ \\
\hline
\end{tabular}

a summary of the experimental design we utilized for ChangeStyle.

We show analyst performance in two ways: graphical and tabular. The graphical representation is the same as the initial experiment: we plot the accuracy of the initial and final submitted TM in the precision-recall space. However, as initial accuracy is no longer the main control in the experiment, we also tabulate the results. Figure 7.1 shows the performance of all analysts. Table 7.2 shows the results in tabular form.

\subsection{HIPAA}

For the HIPAA dataset we used a subset of combinations that were used with ChangeStyle to allow direct result comparison. In total, we used four different combinations of IR methods, filtering, and filter value. Table 6.2 shows a summary of the experimental design we utilized for the HIPAA dataset.

Figure 7.2 shows the performance of all analysts using this dataset. Table 7.3 


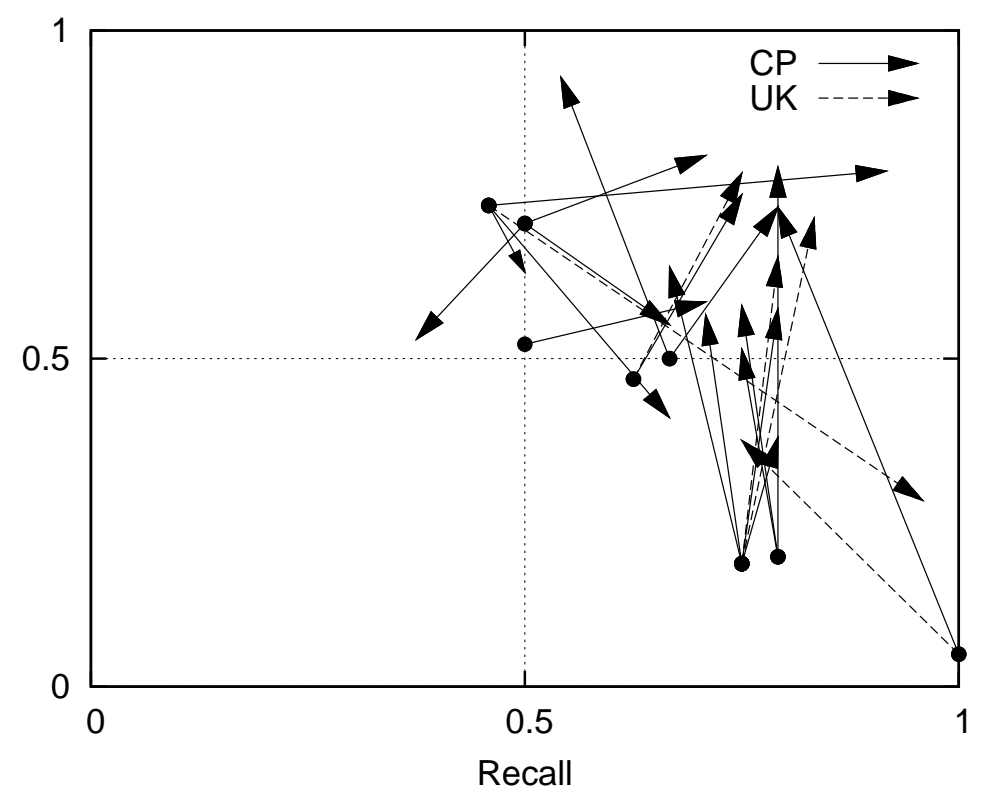

Figure 7.1: Analyst performance when using naturally generated TMs and the ChangeStyle dataset

Table 7.3: Performance for analysts using naturally generated TMs vetting the HIPAA dataset

\begin{tabular}{|c|c|c|c|c|c|c|c|c|c|}
\hline $\begin{array}{l}\text { UserID } \\
\text { UserHA }\end{array}$ & $\begin{array}{l}\text { IR Method } \\
\text { TF-IDF }\end{array}$ & $\begin{array}{l}\text { Filter Type } \\
\text { None }\end{array}$ & $\begin{array}{l}\text { Filter Value } \\
\text { NA }\end{array}$ & $\begin{array}{l}\text { Initial Precision } \\
6.8 \%\end{array}$ & $\begin{array}{l}\text { Initial Recall } \\
81.3 \%\end{array}$ & $\begin{array}{l}\text { Initial } f_{2} \\
25.5 \%\end{array}$ & $\begin{array}{l}\text { Final Precision } \\
60.0 \%\end{array}$ & $\begin{array}{l}\text { Final Recall } \\
56.3 \%\end{array}$ & $\begin{array}{l}\text { Final } f_{2} \\
57.0 \%\end{array}$ \\
\hline UserHB & TF-IDF & None & NA & $6.8 \%$ & $81.3 \%$ & $25.5 \%$ & $21.6 \%$ & $68.8 \%$ & $47.8 \%$ \\
\hline UserHC & Okapi & Value & 0.25 & $57.1 \%$ & $25.0 \%$ & $28.2 \%$ & $52.4 \%$ & $68.8 \%$ & $74.7 \%$ \\
\hline UserHD & Okapi & Value & 0.25 & $57.1 \%$ & $25.0 \%$ & $28.2 \%$ & $41.9 \%$ & $81.3 \%$ & $68.4 \%$ \\
\hline UserHE & Okapi & Local & 1 & $40.0 \%$ & $25.0 \%$ & $27.0 \%$ & $33.3 \%$ & $31.3 \%$ & $31.7 \%$ \\
\hline UserHF & Okapi & Local & 1 & $40.0 \%$ & $25.0 \%$ & $27.0 \%$ & $80.0 \%$ & $25.0 \%$ & $29.0 \%$ \\
\hline UserHG & TF-IDF & Local & 3 & $26.7 \%$ & $50.0 \%$ & $42.6 \%$ & $62.5 \%$ & $62.5 \%$ & $62.5 \%$ \\
\hline UserHH & TF-IDF & Local & 3 & $26.7 \%$ & $50.0 \%$ & $42.6 \%$ & $57.1 \%$ & $50.0 \%$ & $51.3 \%$ \\
\hline
\end{tabular}

shows the analyst performance in tabular form.

\subsection{IR method Used}

The first research question posed was about the impact of the IR method on the final TMs accuracy? We wanted to examine and determine if analysts would be more successful using TF-IDF or Okapi. We show a direct comparison using the same filtering technique and values but different IR method. There are 8 TFIDF and 9 Okapi datapoints which meet this criteria. Figure 7.3(a) shows the 


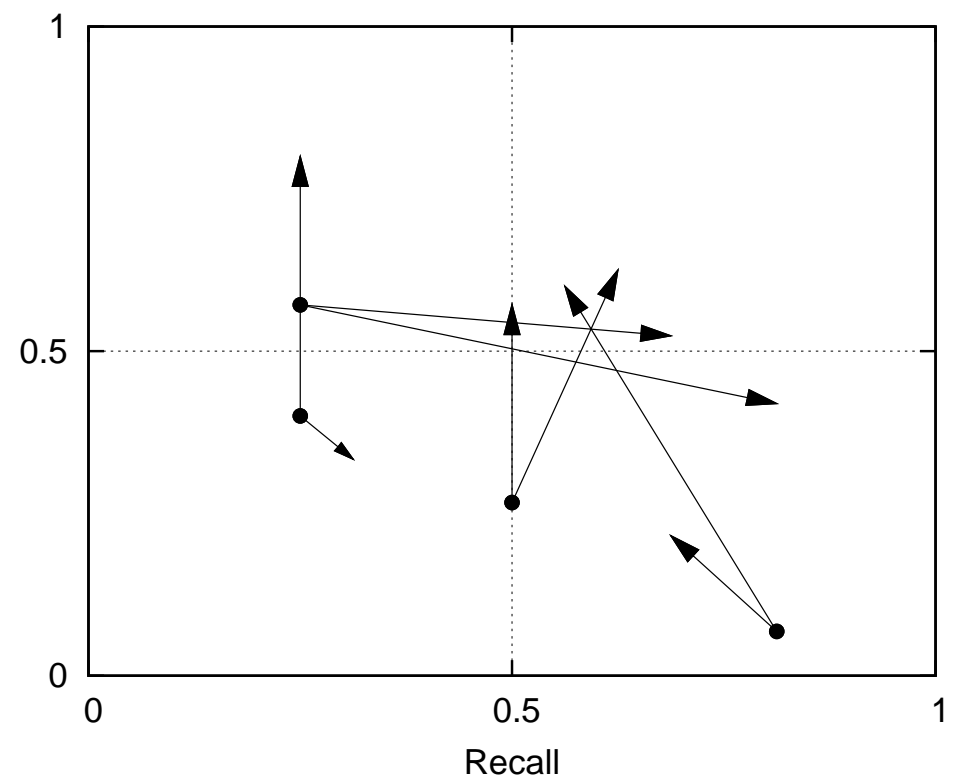

Figure 7.2: Analyst performance when using naturally generated TMs and the HIPAA dataset

performance of analysts using TF-IDF and Figure 7.3(b) shows the performance of analysts using Okapi. Table 7.4 shows the performance in tabular form that meet this criteria.

Analyzing these figures and table shows similar analyst behavior in each filter option. Examining the analyst behavior it appears those utilizing Okapi made

Table 7.4: Comparing analyst performance when using TF-IDF and Okapi generated TMs while vetting the ChangeStyle dataset

\begin{tabular}{|c|c|c|c|c|c|c|c|c|c|}
\hline UserID & IR Method & Filter Type & Filter Value & Initial Precision & Initial Recall & Initial $f_{2}$ & Final Precision & Final Recall & Final $f_{2}$ \\
\hline UserC & Okapi & Value & 0.25 & $73.3 \%$ & $45.8 \%$ & $49.6 \%$ & $28.4 \%$ & $95.8 \%$ & $65.0 \%$ \\
\hline UserD & Okapi & Value & 0.25 & $73.3 \%$ & $45.8 \%$ & $49.6 \%$ & $78.6 \%$ & $91.7 \%$ & $88.7 \%$ \\
\hline$\overline{\text { UserE }}$ & Okapi & Value & 0.25 & $73.3 \%$ & $45.8 \%$ & $49.6 \%$ & $41.0 \%$ & $66.7 \%$ & $59.3 \%$ \\
\hline$\overline{\text { UserF }}$ & Okapi & Value & 0.25 & $73.3 \%$ & $45.8 \%$ & $49.6 \%$ & $63.2 \%$ & $50.0 \%$ & $52.2 \%$ \\
\hline UserG & TF-IDF & Value & 0.25 & $70.6 \%$ & $50.0 \%$ & $53.1 \%$ & $55.2 \%$ & $66.7 \%$ & $64.0 \%$ \\
\hline UserH & TF-IDF & Value & 0.25 & $70.6 \%$ & $50.0 \%$ & $53.1 \%$ & $52.9 \%$ & $37.5 \%$ & $39.8 \%$ \\
\hline UserI & TF-IDF & Value & 0.25 & $70.6 \%$ & $50.0 \%$ & $53.1 \%$ & $81.0 \%$ & $70.8 \%$ & $72.7 \%$ \\
\hline UserJ & Okapi & Local & 1 & $46.9 \%$ & $62.5 \%$ & $58.6 \%$ & $78.3 \%$ & $75.0 \%$ & $75.6 \%$ \\
\hline UserK & Okapi & Local & 1 & $46.9 \%$ & $62.5 \%$ & $58.6 \%$ & $75.0 \%$ & $75.0 \%$ & $75.0 \%$ \\
\hline UserL & TF-IDF & Local & 1 & $50.0 \%$ & $66.7 \%$ & $62.5 \%$ & $73.1 \%$ & $79.2 \%$ & $77.9 \%$ \\
\hline UserM & TF-IDF & Local & 1 & $50.0 \%$ & $66.7 \%$ & $62.5 \%$ & $92.9 \%$ & $54.2 \%$ & $59.1 \%$ \\
\hline UserQ & TF-IDF & Global & 96 & $18.8 \%$ & $75.0 \%$ & $46.9 \%$ & $64.0 \%$ & $66.7 \%$ & $66.1 \%$ \\
\hline UserR & TF-IDF & Global & 96 & $18.8 \%$ & $75.0 \%$ & $46.9 \%$ & $57.6 \%$ & $79.2 \%$ & $73.6 \%$ \\
\hline UserS & TF-IDF & Global & 96 & $18.8 \%$ & $75.0 \%$ & $46.9 \%$ & $56.7 \%$ & $70.8 \%$ & $67.5 \%$ \\
\hline UserT & Okapi & Global & 96 & $19.8 \%$ & $79.2 \%$ & $49.5 \%$ & $58.1 \%$ & $75.0 \%$ & $70.9 \%$ \\
\hline UserU & Okapi & Global & 96 & $19.8 \%$ & $79.2 \%$ & $49.5 \%$ & $51.4 \%$ & $75.0 \%$ & $68.7 \%$ \\
\hline UserV & Okapi & Global & 96 & $19.8 \%$ & $79.2 \%$ & $49.5 \%$ & $79.2 \%$ & $79.2 \%$ & $79.2 \%$ \\
\hline
\end{tabular}


larger changes than those using TF-IDF, both when they improved and when they degraded the accuracy of the TM. An example of this can be seen from the analysts performing value filtering (70\% precision and 50\% recall for TFIDF, $73 \%$ precision and $46 \%$ recall for OKAPI). TF-IDF tended to have larger changes in precision and recall compared to Okapi. For two of the four analysts, this change resulted in larger decreases in accuracy.

This finding is also visualized in Figures 7.3(c) and 7.3(d). As seen here, while Okapi has a few larger deltas, the majority of both IR methods tend to fall in the same $f_{2}$-measure range, between .6 and .75 range.

We conclude that from our pilot study the choice of IR method does not seem to have a significant impact on the analyst ability to perform tracing. At present, we do not have enough datapoints to conduct statistical significance tests. We plan on conducting this in the future once we obtain results from additional participants.

\subsection{Filtering Method Used}

The second research question posed was how does the filtering method used impact the analysts performance while producing a final TM. We show the analyst performance using each of the six filters used. Figures 7.4(a) shows no filtering, 7.4(b) shows value filtering, 7.5(a) shows local top 1 filtering, 7.5(b) shows local top 3 filtering, 7.6(a) shows global top 23 filtering, and 7.6(b) shows global top 96 filtering. Table 7.2 shows the performance for all the analysts vetting using the different filtering techniques.

Each filter type showed a distinctive behavior. 

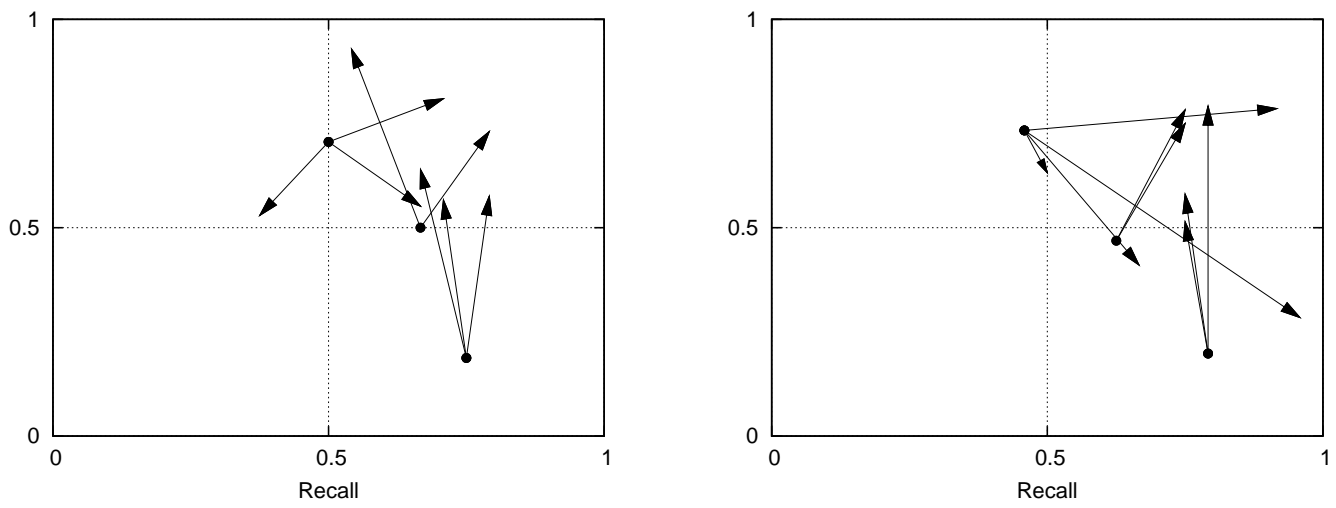

(a) Analyst performance for analysts using TF- (b) Analyst performance for analysts using IDF Okapi

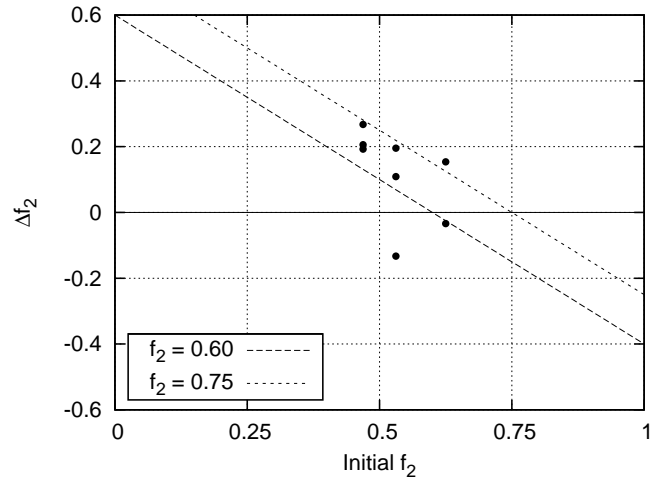

(c) Change in $f_{2}$-measure for TF-IDF

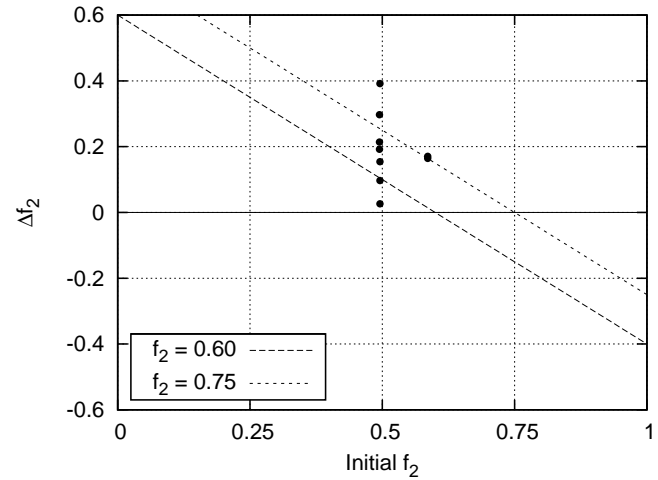

(d) Change in $f_{2}$-measure for Okapi

Figure 7.3: Comparing analyst performance based on IR method differences. (a) shows analysts using TF-IDF and (b) shows analysts using Okapi.

No Filter. Two participants were assigned TMs without a filter applied. The performance for these participants is shown in Figure 7.4(a). Both participants greatly increased precision but at the cost of recall. The TMs generated without being filtered where very large, 487 links, with many false positives, 463 false links. Because of this number of false positives, the users focused solely on correcting errors of commission.

As a consequence, the analysts successfully removed the majority of false links, 433 in one case and 456 in the other. However, during this process these 


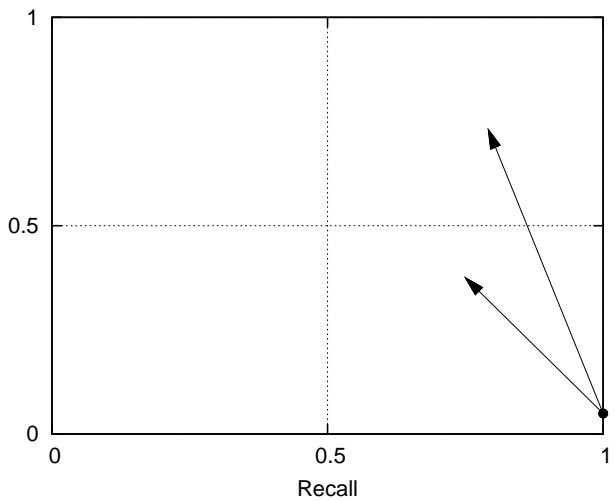

(a) Analyst performance for analysts using no filtering

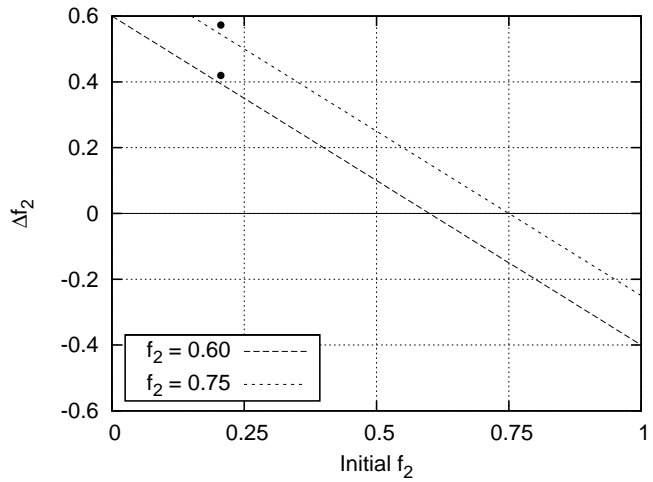

(c) Change in $f_{2}$-measure for no filtering

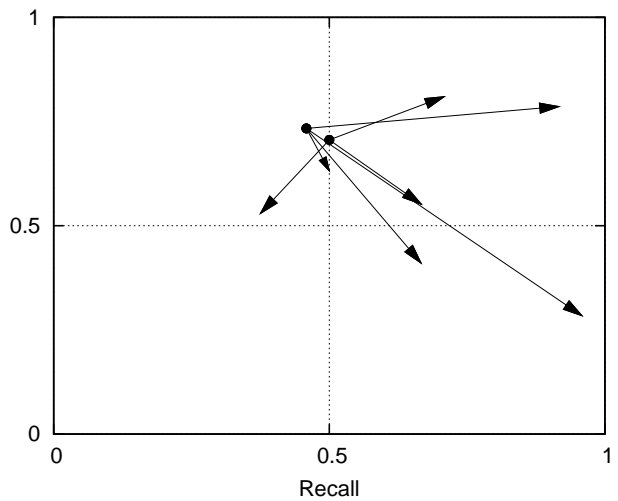

(b) Analyst performance for analysts using value filtering

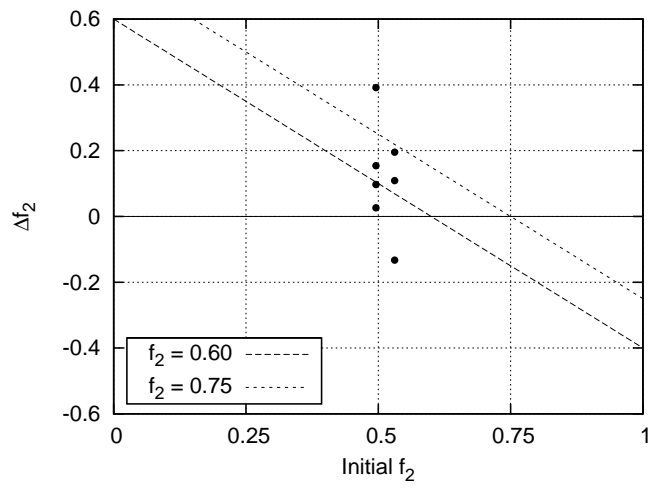

(d) Change in $f_{2}$-measure for value filtering

Figure 7.4: Comparing analyst performance based on filtering technique. (a) and (c) show analyst performance using no filtering, and (b) and 7.4(d) show analyst performance using value filtering.

analysts also removed a few correct links, 5 and 6 respectively. Examining the analyst performance based on decision correctness, these analysts achieved the highest proportion of correct decisions, at worst case 36 incorrect decisions out of 487 candidate decisions. This calculates to be $93 \%$ of all decisions where correct.

The problem we see with this case is that these analysts were presented with 90\% of all link possibilities as recommended. This task then turns into a validation task very similar to manual validation without computer assistance. Because the analyst validates so many links they inherently make some incorrect judge- 


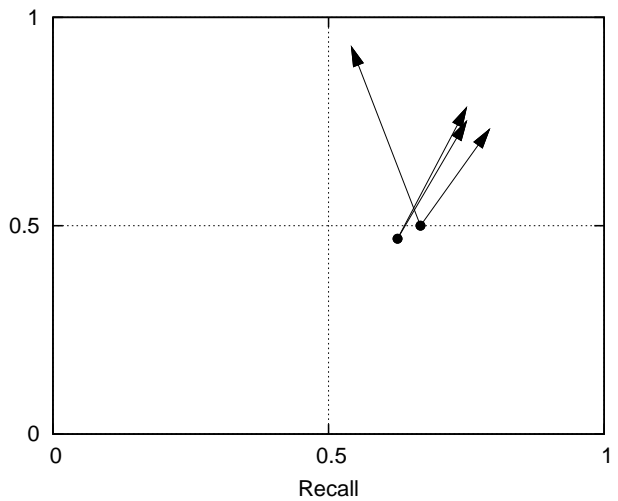

(a) Analyst performance for analysts using local top 1 filtering

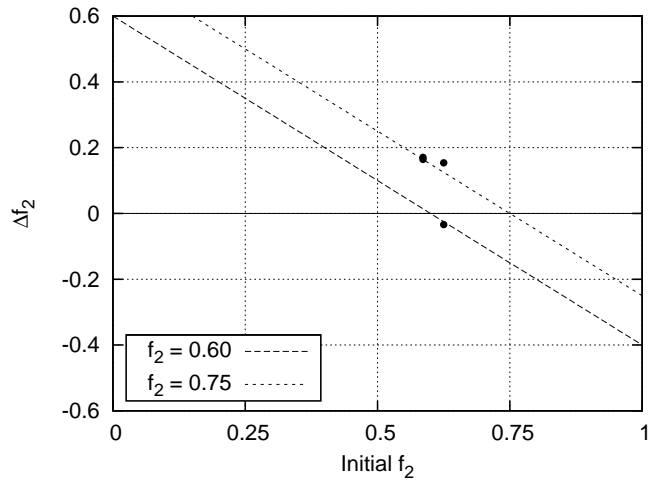

(c) Change in $f_{2}$-measure for local top 1 filter- (d) Change in $f_{2}$-measure for local top 3 filtering

Figure 7.5: Comparing analyst performance based on filtering technique. (a) and (c) show analyst performance using local top one filtering, and (b) and 7.5(d) show analyst performance using local top three filtering.

ments and remove valid links that should be left. Because of these problems, presenting TMs without filtering should not be done.

Value Filter. We had seven participants assigned TMs with value filtering using a 0.25 threshold value. The performance for these participants is shown in Figure 7.4(b). Participants with TMs using this filter resulted in the least consistent results. However, similar patterns still emerge. 
All but one analyst increased their recall, although to varying degrees. In the majority of the cases (4 out of 7) precision decreased. We look at analyst performance utilizing $f_{2}$-measure. Figure $7.4(\mathrm{~d})$ shows the change in $f_{2}$-measure compared to the initial $f_{2}$-measure. From this figure we see that only one analyst submitted a final TM with the $f_{2}$-measure above 0.75 . Additionally three out of seven analysts were not able to reach the $0.6 f_{2}$-measure mentioned by Cuddeback et al.[6].

When we observe the average trend from these analysts region, they tend to improve recall, decrease precision, and end with an $f_{2}$-measure around or just below 0.60. The TMs generated using these filters have half the true links and very few false positives. The analysts from these TMs focus on searching for errors of omission. They tend to locate several of the omitted links, however in the process introduce several false positives. In fact, when examining the two individuals who increased precision, this increase was an artifact of introducing several correct links leading to a higher precision.

Due to the overall behavior trend we feel that the use of value filtering may be difficult for analysts to consistently improve overall accuracy. An additional problem with value filtering is that it is highly sensitive to the threshold value. We plan to experiment with different threasholds in our future work.

Local Filter. We had seven participants assigned TMs generated with the help of local filtering. These filters were broken down to four using local top one filtering while the other three participants used local top three filtering. The performance for these participants is shown in Figure 7.5(a) for local top one and Figure 7.5(b) for local top three filtering.

While we notice small differences between local top one and top three filtering, 
in general similar patterns appear. In all but one case the analysts improved precision and recall. Analysts receiving top one filters improved both precision and recall by approximately the same amount. The participants who received top three filtered results improved the precision by a significant amount more than recall. Even with these changes both local filters ended up around the same final precision and recall values on average.

All but one participants receiving local filtered TMs improved their final accuracy when measured by $f_{2}$-measure. All participants submitted final TMs with the $f_{2}$-measure of at least 0.60 . In fact, five of the seven participants submitted final TMs with a $f_{2}$-measure greater than 0.75 , including the second highest accuracy TM submitted in our study.

These participants performed the best of any in our study. We feel this positive increase in both precision and recall is the ideal behavior we look for. Additionally, we feel local filtering is the least tied to the dataset of any filtering options. While some datasets may have multiple links between high and low elements, and other have few, the fact that local filtering pulls out the best candidate links for validation for each high-level element. Even though the ideal filter value may vary from one dataset to another, the fact that analysts were able to preform well with local top one filter indicates even with a low filter value candidates can still preform vetting successfully.

Global Filter. We had seven participants assigned TMs with global filtering. These filters were broken down to six using global top 96 filtering and one participant using global top 23 filtering. The performance for these participants is shown in Figure 7.6(a) for top 23 filtering and Figure 7.6(b) for top 96 filtering.

We notice different behavior between global top 23 and 96 filtering. In global 

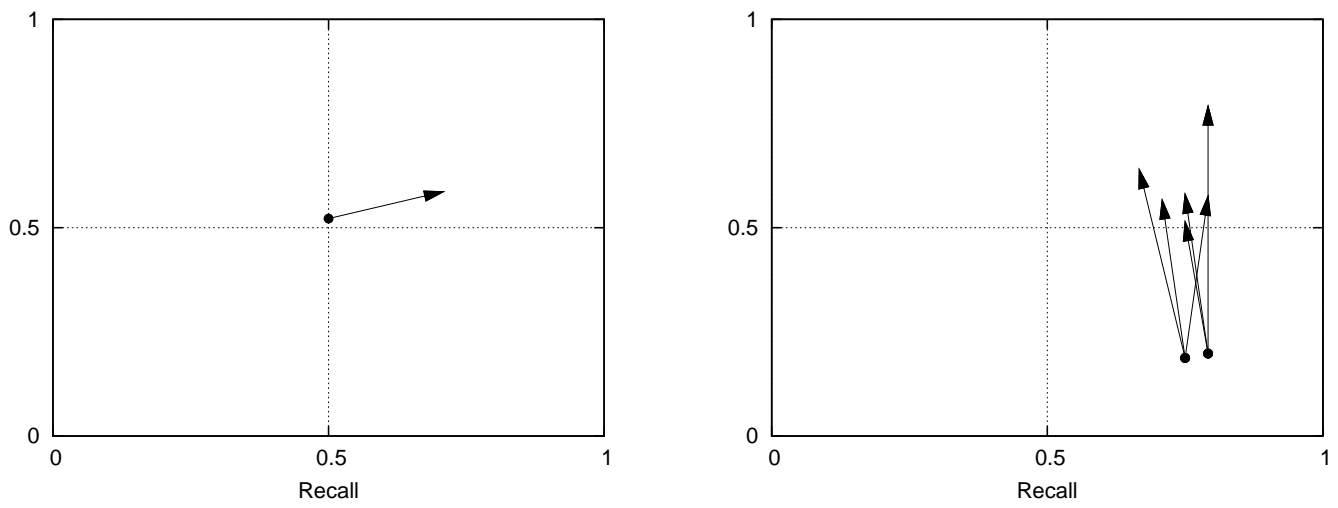

(a) Analyst performance for analysts using (b) Analyst performance for analysts using global top 23 filtering

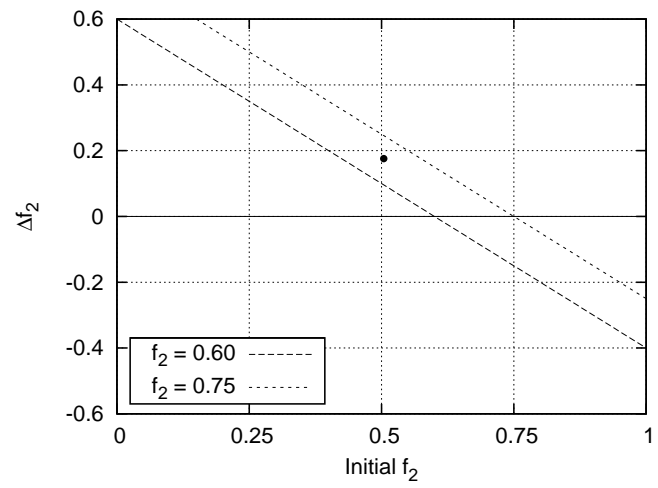
global top 96 filtering

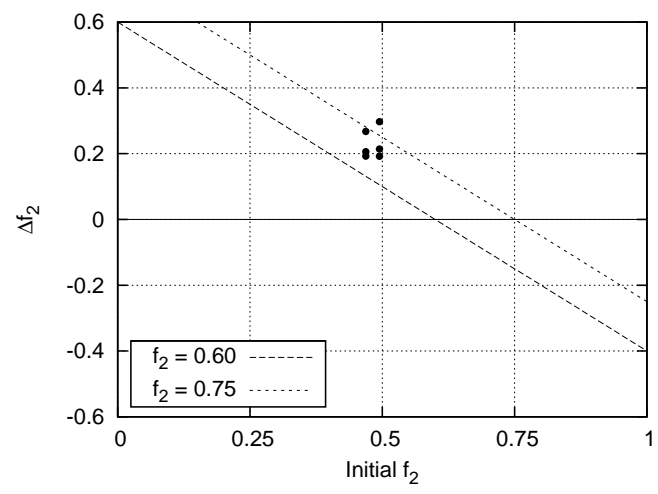

(c) Change in $f_{2}$-measure for global top 23 fil- (d) Change in $f_{2}$-measure for global top 96 filtering tering

Figure 7.6: Comparing analyst performance based on filtering technique. (a) and (c) show analyst performance using global top 23 filtering, and (b) and 7.6(d) show analyst performance using global top 96 filtering.

top 23 filtering the participant focused solely on locating errors of omission, successfully adding a few links without introducing any false positives, leading to an increase in recall with a smaller, indirect increase in precision.

In global top 96 filtering on the other hand participants focused mainly on correcting errors of commission. These analysts removed several false positives with many also removing one or two correct links. This behavior led to analysts increasing their precision with a minor change in precision. 
Even though these differences are apparent, the final TMs from global filtering participants were fairly similar. All but one participant submitted final TMs with a $f_{2}$-measure between 0.60 and 0.75 with the main cluster of analysts submitting with $f_{2}$-measure around 0.70. This behavior is seen in Figures 7.6(c) and 7.6(d) of change in $f_{2}$-measure for global top 23 and 96 filters respectively. The one participant submitting a TM with $f_{2}$-measure greater than 0.75 showed a similar behavior as the other analysts however, they where successful in identifying true links when removing links to correct errors of commission.

Global filtering appears to present TMs which analysts can successfully improve in accuracy. One main problem with global filtering is what filter value to use. As displayed by the two global filter options, how many top links to display to the user makes a large impact on the results. While not shown as significantly as possible, a global filter set too low could lead to very small TMs forcing analysts to focus solely on errors of omission. However, if the filter value is set too high, the TM may end up having large amounts of false positives. Due to these potential problems, we feel applying global filters may be problematic without the careful choice of filter value.

\subsection{ChangeStyle vs HIPAA Analyst Behavior}

We wanted to examine the analyst behavior using multiple datasets and determine if our observations are general or specific to ChangeStyle. To accomplish this we ran a smaller version of our pilot study using eight students at Cal Poly. An overview of the experimental setup is shown in Table 6.2.

We utilized the same IR and filtering techniques for both datasets. It should be noted that due to the differences in the datasets the initial TMs accuracies 


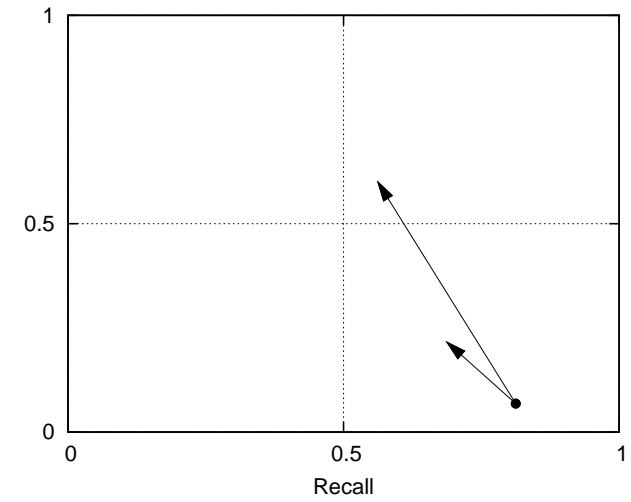

(a) HIPAA Analyst performance for analysts using no filtering

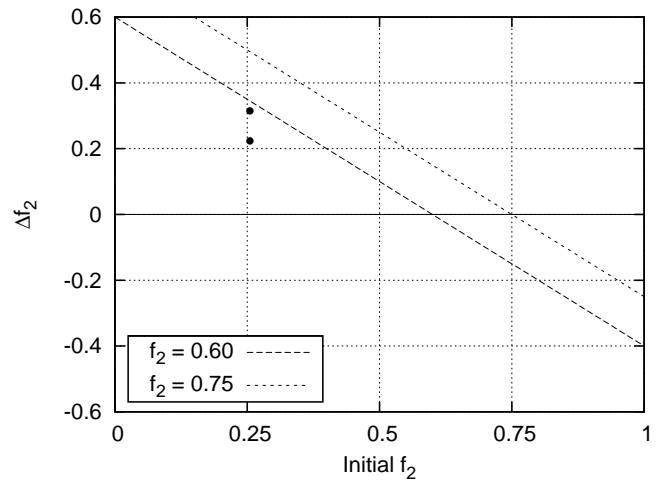

(c) Change in $f_{2}$-measure for HIPAA no filtering

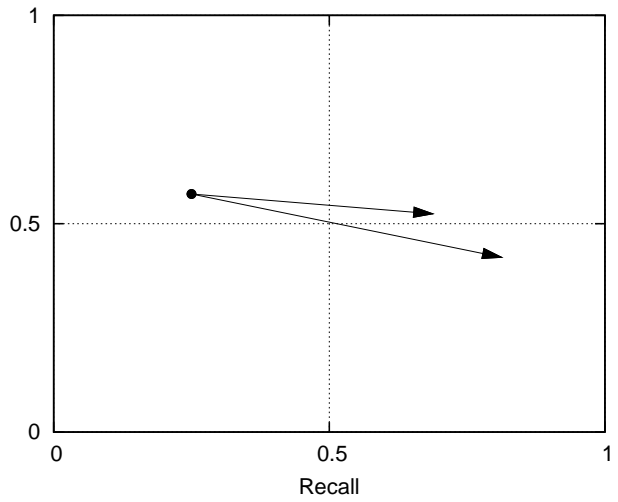

(b) HIPAA Analyst performance for analysts using value filtering

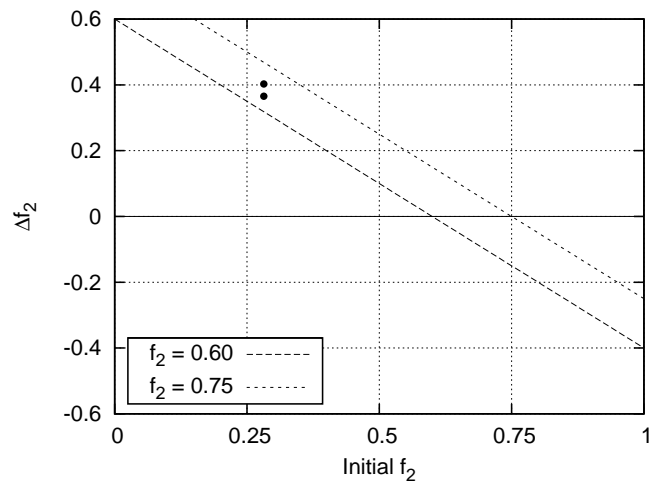

(d) Change in $f_{2}$-measure for HIPAA value filtering

Figure 7.7: Comparing analyst performance when vetting HIPAA based on filtering technique. (a) and (c) show analyst performance using no filtering, and (b) and $7.7(\mathrm{~d})$ show analyst performance using value filtering.

are different for TMs generated using the same process. Due to these differences when examining the behavior we examine the changes not the raw accuracy end points.

No Filter. We had two HIPAA participants assigned TMs with out a filter applied. The performance for these participants is shown in Figure 7.7(a). Both of these participants increased precision at the cost of decreasing recall. This behav- 
ior is very similar as the behavior shown by the analysts vetting the ChangeStyle dataset when the TMs where not filtered. The main difference between analyst behavior can be seen when comparing the $f_{2}$-measures. In the ChangeStyle experiment, $f_{2}$-measure increased by at least 0.4 , while the analysts vetting the HIPAA dataset only increased $f_{2}$-measure by 0.2 and 0.3 .

This change in final TM accuracy can be explained by taking the initial TM accuracy in to account. Since with the HIPAA datasets the IR methods where not able to obtain all true links, the initial candidate TMs had about the same precision but much lower recall. In both datasets analysts increased precision and decreased recall by about the same amount. Despite this difference, overall analyst behavior appears to be consistent when not utilizing filtering.

Value Filter. We had two HIPAA participants assigned TMs using value filtering. The performance for these participants is shown in Figure 7.7(b). Both participants increased recall fairly significantly but at the cost of precision. These analysts focused on correcting errors of omission and while they added several correct links, they also added a few false positives.

This behavior is consistent with the analyst behavior for vetting value filtering on the ChangeStyle dataset. These analysts produced candidate TMs with $f_{2^{-}}$ measure of around 0.7. This is also consistent with the behavior seen in the ChangeStyle study which had the largest grouping of analysts around $f_{2}$-measure of 0.7 .

Local Filter We had four HIPAA participants assigned TMs using local filtering. This was split into two analysts using top one filtering and two analysts using top three filtering. The performance for these participants is shown in 
Figure 7.8(a) for top one filtering and Figure 7.8(b) for top three filtering.

Three of the four analysts increased precision. Two of these analysts solely focused on eliminating false positives, maintaining their initial recall. These analysts did not utilize the "all" tab at all to search for any missing links. The remaining two analysts increased their recall, adding one and two additional links.

This behavior showed some similarities to the analysts vetting the ChangeStyle dataset using local filtering. Like ChangeStyle, the HIPAA analysts where able to eliminate several false positives. However, unlike the ChangeStyle analysts, the HIPAA analysts did not search out missing links and instead seemed content with the candidate links presented. We are unsure why this difference appeared, however we believe it may be a lack of understanding about the use of the "all" tab to locate missing links. Were the analysts to have utilized the "all" tab, it is likely that they would have located missing links and displayed similar behavior to the ChangeStyle analysts. 

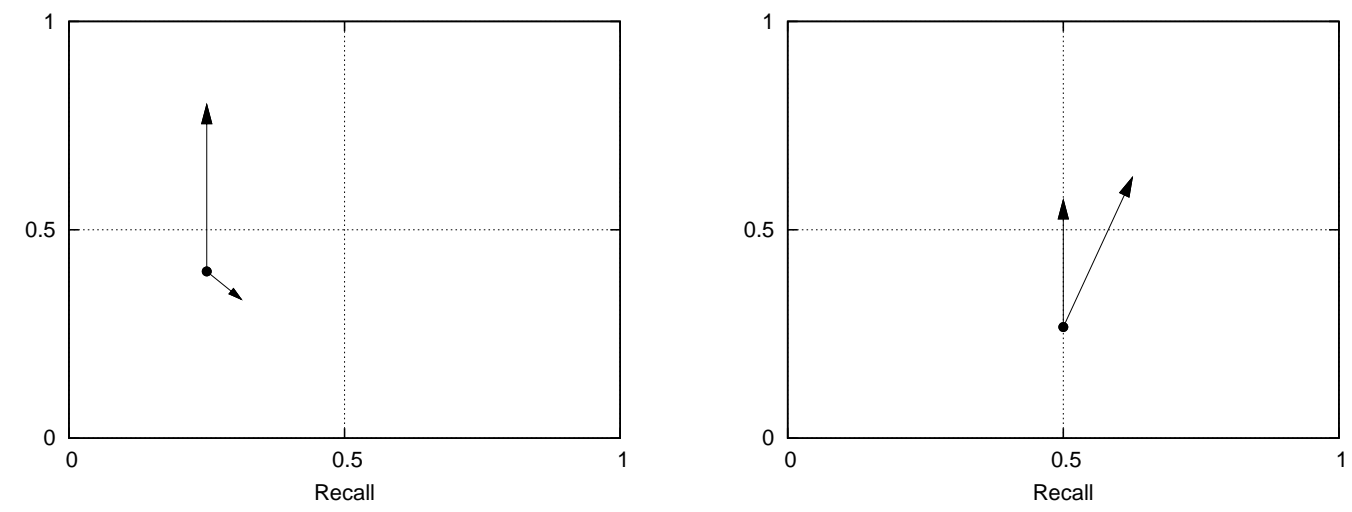

(a) HIPAA Analyst performance for analysts (b) HIPAA Analyst performance for analysts using local top 1 filtering using local top 3 filtering
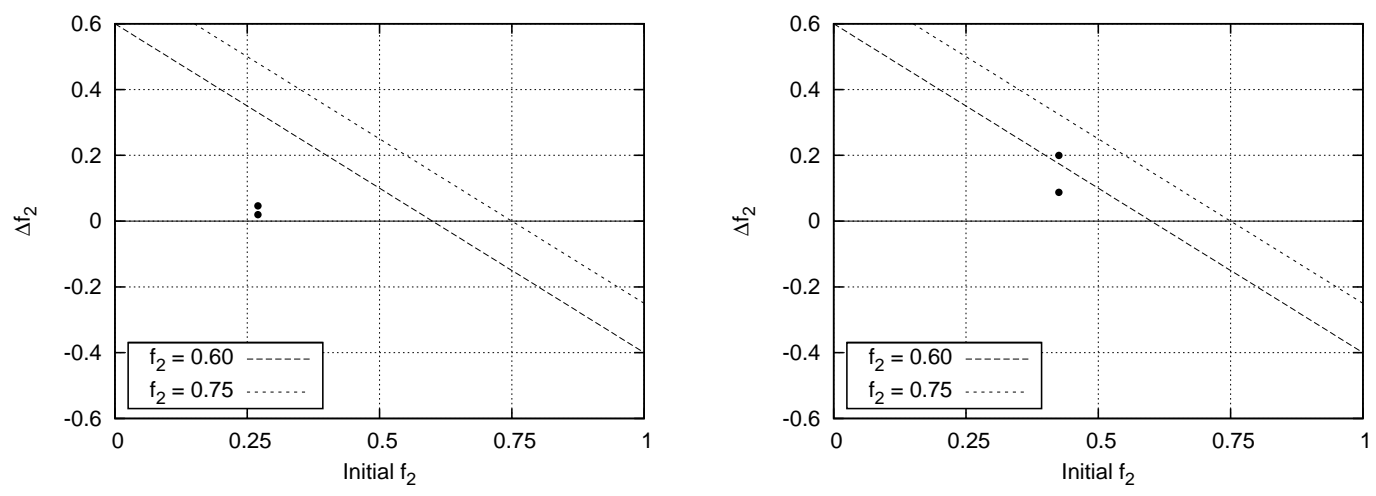

(c) Change in $f_{2}$-measure for HIPAA local top (d) Change in $f_{2}$-measure for HIPAA local top 1 filtering

3 filtering

Figure 7.8: Comparing analyst performance when vetting HIPAA based on filtering technique. (a) and (c) show analyst performance using local top one filtering, and (b) and 7.8(d) show analyst performance using local top three filtering. 


\section{Chapter 8}

\section{Future Work}

While we addressed many of the limitations of Cuddeback et al.[6], there are still many directions in which we plan to expand our research. One of the primary areas is to continue to conduct experiments using naturally generated TMs. We plan on expanding our pilot study with more participants. In addition, we plan on incorporating the other baseline variables from our research design, user feedback and different user interfaces.

With these additional data points this will allow us to conduct a second statistical analysis to determine the impact of the new baseline independent variables and attempt to see which variables have the largest impact on the dependent variables. This will allow us to apply this knowledge to assist in creating a morewidely usable traceability tool.

Second, while we were able to expand our study using a second dataset, we would like to gather more data from this dataset. Additionally, we would like to expand our study to utilize additional datasets. While good traceability datasets are scarce, one of the goals of TraceLab is to generate community- 
accepted datasets and benchmarks. We plan on utilizing these datasets in addition to our datasets. This expansion will allow us to conduct further analysis to assist in our goal of creating a universal semi-automated traceability tool.

Finally, we would like to automate the data collection and analysis. Currently, much of our analysis requires extensive manual processing such that as new TMs are submitted scripts must be manually run. One solution to this is to create modules to interact in TraceLab[3]. Fortunately, our experimental design and the current implementation of RETRO.net are modularized, this still will require these modules to be ported to the TraceLab framework as well as convert our current analysis tools to TraceLab.

An additional benefit to converting to a TraceLab-based experiment is to allow cross-university experiments. Using TraceLab, we can easily store a player version of our experiment such that any universities who wish to participate could load the player and contribute the results back for automatic analysis. We feel this is the ideal scenario to allow a wide participant base which will allow the most productive statistical analysis. 


\section{Chapter 9}

\section{Conclusions}

This thesis looks to continue where the study of Cuddeback et al.[6] left off. We initially undertook a study which expanded upon [6] by utilizing a second software traceability tool and additionally by asking analysts to vet candidate TMs manually. We added 51 additional datapoints which we combined with the results presented by Cuddeback et al. This dataset allowed us to conduct a more thorough analysis including running statistical analysis to examine which variables have impact on analysts decisions.

Using this larger data sample we were able to confirm key findings of Cuddeback et al., both informally and formally. In particular we found that the largest impact on the analysts' final TM accuracy was the initial TM accuracy. Additionally, we found only one other variable, ValidEff, which showed any statistically significant influence on the accuracy of the final TM. However, this influence was counter-productive, it showed the analysts' who put in more effort into validating candidate links resulted in lower final TM accuracy.

Upon confirming the findings of Cuddeback et al., we expanded upon their 
findings by creating a new, simple and repeatable experimental framework. This framework utilizes naturally generated TMs and examines how analysts interact with these TMs. We conduct a pilot study utilizing this framework at two locations and using two different datasets.

Based on the results from this pilot study we observe that the IR method used to generate TMs does not appear to have significant impact on the analysts' vetting accuracy. In addition we present and examine several filtering techniques. We show that not filtering IR results leads to poor analyst behavior. In addition, we show both global and local filtering are promising with analysts consistently improving their TMs accuracy.

We show that local filtering leads to the best analyst behavior on average. In addition we conjecture that local filtering can move from one dataset to another successfully without suffering significant impact from different filter values. Additionally, our pilot shows that utilizing the same IR and filtering techniques on different datasets results in similar analyst behaviors. This was a key finding as previously Cuddeback et al. had only applied the experiment to one dataset. Expanding to multiple datasets and showing similar trends provides additional support that our findings are applicable to multiple datasets rather than specific to a single one.

Finally, we provide evidence that analysts show similar behavior trends while vetting artificially and naturally generated TMs. While there are smaller differences between the overall analyst behavior, the trends on a whole appear to hold true. This provides additional support to our key claim, with semi-automated tracing we can no longer rely solely on the accuracy of the automated methods as the criteria for the quality of the tracing process. In contrast, we must now acknowledge the human analyst as a vital component 
in the tracing process! We must utilize this new knowledge and search for new IR, filtering, and delivery methods in order to create semi-automated tracing systems which can account for and mitigate human fallible behavior. 


\section{Bibliography}

[1] Health insurance portability and accountability act of 1996, 1996.

[2] G. Antoniol, G. Canfora, G. Casazza, A. De Lucia, and E. Merlo. Recovering traceability links between code and documentation. IEEE Transactions on Software Engineering, 28(10):970-983, Oct 2002.

[3] J. Cleland-Huang, A. Czauderna, A. Dekhtyar, O. Gotel, J. Hayes, E. Keenan, G. Leach, J. Maletic, D. Poshyvanyk, Y. Shin, et al. Grand Challenges, Benchmarks, and TraceLab: Developing Infrastructure for the Software Traceability Research Community. In TEFSE '11: Proceedings of the 5th International Workshop on Traceability in Emerging Forms of Software Engineering., 2011.

[4] J. Cleland-Huang, A. Czauderna, M. Gibiec, and J. Emenecker. A machine learning approach for tracing regulatory codes to product specific requirements. In Proceedings of the 32nd ACM/IEEE International Conference on Software Engineering-Volume 1, pages 155-164. ACM, 2010.

[5] J. Cleland-Huang, R. Settimi, O. BenKhadra, E. Berezhanskaya, and S. Christina. Goal-centric traceability for managing non-functional requirements. In ICSE '05: Proceedings of the 27th international conference on Software engineering, pages 362-371, New York, NY, USA, 2005. ACM. 
[6] D. Cuddeback. Automated requirements traceability: the study of human analysts. Master's Theses and Project Reports, May 2010.

[7] D. Cuddeback, A. Dekhtyar, and J. H. Hayes. Automated requirements traceability: the study of human analysts. In Proceedings of the 18th International Conference on Requirements Engineering, 2010., pages 94-101. IEEE, Sept. 2010.

[8] A. De Lucia, R. Oliveto, and G. Tortora. Adams re-trace: traceability link recovery via latent semantic indexing. In Proceedings of the 30th international conference on Software engineering, pages 839-842. ACM, 2008.

[9] S. Deerwester, S. Dumais, G. Furnas, T. Landauer, and R. Harshman. Indexing by latent semantic analysis. Journal of the American society for information science, 41(6):391-407, 1990.

[10] A. Dekhtyar, O. Dekhtyar, J. Holden, J. Hayes, D. Cuddeback, and W. Kong. On human analyst performance in assisted requirements tracing: Statistical analysis. In Proceedings of the 19th International Conference on Requirements Engineering, 2011., to appear. IEEE, Sept. 2011.

[11] A. Dekhtyar, J. H. Hayes, and J. Larsen. Make the most of your time: How should the analyst work with automated traceability tools? In PROMISE '0\%: Proceedings of the Third International Workshop on Predictor Models in Software Engineering, page 4, Washington, DC, USA, 2007. IEEE Computer Society.

[12] M. Eaddy, A. Aho, G. Antoniol, et al. Cerberus: Tracing requirements to source code using information retrieval, dynamic analysis, and program 
analysis. In The 16th IEEE International Conference on Program Comprehension, pages 53-62. IEEE, 2008.

[13] A. Egyed, F. Graf, and P. Grunbacher. Effort and Quality of Recovering Requirements-to-Code Traces: Two Exploratory Experiments. In 2010 18th IEEE International Requirements Engineering Conference, pages 221-230. IEEE, 2010.

[14] O. Gotel and C. Finkelstein. An analysis of the requirements traceability problem. In Proceedings of the First International Conference on Requirements Engineering, 1994., pages 94-101. IEEE, Apr. 1994.

[15] J. Hayes, A. Dekhtyar, and J. Osborne. Improving requirements tracing via information retrieval. In Proceedings of the 11th IEEE International Requirements Engineering Conference, 2003., pages 138-147. IEEE, Sept. 2003.

[16] J. Hayes, A. Dekhtyar, and S. Sundaram. Advancing candidate link generation for requirements tracing: the study of methods. IEEE Transactions on Software Engineering., 32(1):4-19, Jan. 2006.

[17] J. Hayes, A. Dekhtyar, S. Sundaram, and S. Howard. Helping analysts trace requirements: An objective look. In Proceedings of the 12th IEEE International Requirements Engineering Conference, 2004., pages 249-259. IEEE, Sept. 2004.

[18] J. H. Hayes and A. Dekhtyar. Humans in the traceability loop: Can't live with 'em, can't live without 'em. In TEFSE '05: Proceedings of the 3rd International Workshop on Traceability in Emerging Forms of Software Engineering., pages 20-23, New York, NY, USA, 2005. ACM. 
[19] J. H. Hayes, A. Dekhtyar, and S. Sundaram. Text mining for software engineering: How analyst feedback impacts final results. In MSR '05: Proceedings of the 2005 International Workshop on Mining Software Repositories., pages 1-5, New York, NY, USA, 2005. ACM.

[20] J. H. Hayes, A. Dekhtyar, S. Sundaram, A. Holbrook, S. Vadlamudi, and A. April. Requirements tracing on target (retro): Improving software maintenance through traceability recovery. Innovations in Systems and Software Engineering: A NASA Journal, 3(3):193-202, Sep 2007.

[21] T. Hofmann. Probabilistic latent semantic indexing. In Proceedings of the 22nd annual international ACM SIGIR conference on Research and development in information retrieval, pages 50-57. ACM, 1999.

[22] W. Kong, J. Hayes, A. Dekhtyar, and J. Holden. How do we trace requirements? an initial study of analyst behavior in trace validation tasks. Proceedings of Cooperative and Human Aspects of Software Engineering, 2011.

[23] Level 1A (L1A) and Geolocation Processing Software Requirements Specification, SDST-059A, GSFC SBRS, September 11, 1997.

[24] J. Lin, C. C. Lin, J. Cleland-Huang, R. Settimi, J. Amaya, G. Bedford, B. Berenbach, O. B. Khadra, C. Duan, and X. Zou. Poirot: A distributed tool supporting enterprise-wide automated traceability. In Proceedings of the 14th International Conference on Requirements Engineering, 2006, pages 363-364, Los Alamitos, CA, USA, 2006. IEEE.

[25] A. Marcus and J. Maletic. Recovering documentation-to-source-code traceability links using latent semantic indexing. In Proceedings of the 25th Inter- 
national Conference on Software Engineering, 2003., pages 125-135. IEEE, May 2003.

[26] MDP Website, CM-1 Project, http://mdp.ivv.nasa.gov/mdp_glossary.html\#CM1.

[27] MODIS Science Data Processing Software Requirements Specification Version 2, SDST-089, GSFC SBRS, November 10, 1997.

[28] R. Oliveto, M. Gethers, D. Poshyvanyk, and A. De Lucia. On the equivalence of information retrieval methods for automated traceability link recovery. In Program Comprehension (ICPC), 2010 IEEE 18th International Conference on, pages 68-71. IEEE, 2010.

[29] G. Salton and M. McGill. Introduction to modern information retrieval, volume 1. McGraw-Hill New York, 1983.

[30] G. Salton, A. Wong, and C. S. Yang. A vector space model for automatic indexing. Commun. ACM, 18:613-620, November 1975.

[31] H. Zaragoza, N. Craswell, M. Taylor, S. Saria, and S. Robertson. Microsoft Cambridge at TREC-13: Web and HARD tracks. In Proceedings of TREC 2004. Citeseer, 2004.

[32] X. Zou, R. Settimi, and J. Cleland-Huang. Improving automated requirements trace retrieval: a study of term-based enhancement methods. Empirical Software Engineering, 15(2):119-146, 2010. 
Appendix A

\section{Informed Consent Form}


The study of semi-automated requirements traceability

\section{WHY ARE YOU BEING INVITED TO TAKE PART IN THIS RESEARCH?}

You are being invited to take part in a research study about trace sets. You are being invited to participate in this research study because you learned about requirements development in software engineering courses at Cal Poly. It is your choice whether or not you volunteer for this study. If you choose not to participate, there will not be any ramifications and you will have the opportunity to perform a different extra credit project of equal value and equal difficulty. If you take part in this study, you will be one of about 12 people to do so.

\section{WHO IS DOING THE STUDY?}

The people in charge of this study are Dr. Alex Dekhtyar, Professor of Computer Science at California Polytechnic State University in San Luis Obispo, California, and Jeff Holden, graduate student of Computer Science at California Polytechnic State University in San Luis Obispo, California. There may be other people on the research team assisting at different times during the study.

\section{WHAT IS THE PURPOSE OF THIS STUDY?}

By doing this study, we hope to obtain information about analyst decision making when working with computer-generated trace sets.

\section{WHERE IS THE STUDY GOING TO TAKE PLACE AND HOW LONG WILL IT LAST?}

The research procedures will be conducted during a class lab period. The total amount of time you will be asked to volunteer for this study is approximately 2 hours over the 1 week.

\section{WHAT WILL YOU BE ASKED TO DO?}

We are asking you to perform a tracing task. Specific instructions are attached to this form.

\section{ARE THERE REASONS WHY YOU SHOULD NOT TAKE PART IN THIS STUDY?}

We will protect your identity. Only Dr. Dekhtyar will know what analyst worked on a specific trace set. We are not aware of any dangers or other risks of which we need to make you aware. 


\section{WILL YOU BENEFIT FROM TAKING PART IN THIS STUDY?}

As a participant in this study you will receive extra credit as specified by your instructor.. You will not receive any payment for taking part in this study.

\section{WHAT WILL IT COST YOU TO PARTICIPATE?}

There are no costs associated with taking part in this study, except your investments of time and intellect.

\section{DO YOU HAVE TO TAKE PART IN THE STUDY?}

If you decide to take part in the study, it should be because you really want to volunteer. You will not lose any benefits or rights you would normally have if you choose not to volunteer. If you choose not to volunteer, nobody will be notified or made aware of your choice. Only the researchers will be aware of your choice, and they will keep this information confidential. You can stop at any time during the study and still keep the benefits and rights you had before volunteering.

\section{IF YOU DON'T WANT TO TAKE PART IN THE STUDY, ARE THERE OTHER CHOICES?}

If you do not want to be in the study, you may choose to undertake a different extra credit opportunity of equal value and equal difficulty as specified by your instructor, or you may choose not to undertake any extra credit projects.

\section{WHO WILL SEE THE INFORMATION YOU GIVE?}

When we write up the study to share with other researchers, you will not be identified in these written materials. However, there are some circumstances in which we may have to show your information to other people. For example, we may be required to show information which identifies you to people who need to be sure we have done the research correctly; these would be people from such organizations as Cal Poly State University.

We will make every effort to prevent anyone who is not on the research team from knowing that you gave us information, or what that information is. For example, your name will be kept separate from the information you give, and these two things will be stored in different places under lock and key.

\section{CAN YOUR TAKING PART IN THE STUDY END EARLY?}

If you decide to take part in the study, you still have the right to decide at any time that you no longer want to continue. You will not be treated differently if you decide to stop taking part in the study. 


\section{WHAT IF YOU HAVE QUESTIONS?}

Before you decide whether to accept this invitation to take part in the study, please ask any questions that might come to mind now. Later, if you have questions about the study, you can contact the investigator, Dr. Alex Dekhtyar, Professor in the Computer Science

Department of Cal Poly State University at 805-756-2387. If you have any questions about your rights as a research volunteer, contact Steve Davis of the Human Subjects Committee at Cal Poly University at sdavis@calpoly.edu, 756-2754, or Dr. Susan Opava, Dean of Research and Graduate Programs at 756-2754, sopava@calpoly.ed. The Human Subjects Committee home page is http://www.calpoly.edu/ sdavis/human.htm. We will give you a copy of this consent form.

$\overline{\text { Signature of person agreeing to take part in the study }}$ Date

Printed name of person agreeing to take part in the study

Name of person providing information to subject

Date

\section{Participant Copy}




\section{WHAT IF YOU HAVE QUESTIONS?}

Before you decide whether to accept this invitation to take part in the study, please ask any questions that might come to mind now. Later, if you have questions about the study, you can contact the investigator, Dr. Alex Dekhtyar, Professor in the Computer Science Department of Cal Poly State University at 805-756-2387. If you have any questions about your rights as a research volunteer, contact Steve Davis of the Human Subjects Committee at Cal Poly University at sdavis@calpoly.edu, 756-2754, or Dr. Susan Opava, Dean of Research and Graduate Programs at 756-2754, sopava@calpoly.ed. The Human Subjects Committee home page is http://www.calpoly.edu/ sdavis/human.htm. We will give you a copy of this consent form.

Signature of person agreeing to take part in the study Date

$\overline{\text { Printed name of person agreeing to take part in the study }}$

Name of person providing information to subject

Date

\section{Researcher Copy}


Appendix B 


\section{Manual Trace Instructions}

\begin{tabular}{|lll|}
\hline Fall $2010 \quad$ Manual Traceability Experiment \\
\hline
\end{tabular}

Traceability Study Instructions

Thank you for participating in the traceability study. The goal of this study is to assess how human analysts interact with requirement traceability matrices. This document outlines your task for the experiment.

Setup

You will be given a study packet including the requirements for a checkstyle plug-in, test cases to test functionality, as well as a generated candidate matrix.

Individual Work

The task should be completed during the lab period.

The experiment assesses the work of individual analysts decisions when evaluating a candidate traceability matrix. Therefore, it is crucial to the experimental design that while performing the task outlined below you do not interact with other people participating in the experiment.

If you have specific questions about the nature of the task, please feel free to approach any member of the research team administering the experiment. We will try to address all your questions.

\section{The Task}

You are given three artifacts of a software development process:

1. a requirements document for a Java Style Checker ${ }^{1}$

2. a list of test cases for this Style Checker

3. a candidate traceability matrix that links the two.

Your task is to validate the presented traceability matrix. In particular, we ask you to validate the links present in the traceability matrix, and, if necessary, introduce new links to it.

As you are evaluating links please record all the test cases that you consider as well as the test cases you feel meet this requirement on the attached final traceability matrix form. Your final traceability matrix will encompass all requirement/test case matches as recorded on your final form under the validated column.

\section{Post-Task Survey}

The experiment is considered completed after you have submitted the final RTM form and answered the post-experimental survey.

A copy of the survey will be provided to you upon declared completion of the tracing task. When you have completed the tracing task:

- Hand the forms to one of the researchers. They will then give you a post-experiment survey

- Please complete this survey and turn it in to one of the researchers.

If you have insuficiant time to complete the survey during the lab period please bring the completed survey and handin at the next class.

Thank you very much for your participation!

${ }^{1} \mathrm{~A}$ project from a previous CSC 308 course

1 
Appendix C

\section{RETRO.net Tool Instructions}




\begin{tabular}{|lll|}
\hline Spring $2011 \quad$ Automated Traceability Experiment \\
\hline
\end{tabular}

Traceability Study Instructions

Thank you for participating in the traceability study. The goal of this study is to assess how human analysts interact with special-purpose tracing software. This document outlines your task for the experiment.

\section{Setup}

You will be working with a requirements tracing tool called RETRO (Requirements Tracing On-Target). This tool is built on .NET, therefore to run the tool it will be required to have .NET installed on the machine. This should be on all lab machines when loaded in Windows.

To install download the tool from

http://users.csc.calpoly.edu/ jholden/RETROV1.zip

Once the zip is downloaded extract the RETROV1 folder. You can then run the application by running Retro.exe.

To perform the assigned task after launching RETRO log in using the user ID issued to you. In the password field re-type the user ID. Your account is: 


\section{Individual Work}

Use the lab period to work on the task. If you need time beyond the lab period to complete the task, please complete the task later. If you need to switch computers during the task refer to the file-transfer section of this document.

The experiment assesses the work of individual analysts with the software. Therefore, it is crucial to the experimental design that while performing the task outlined below you do not interact with other people participating in the experiment.

If you have specific questions about the nature of the task or the software, please feel free to approach any member of the research team administering the experiment. We will try to address all your questions.

\section{The Task}

You will be using RETRO to trace a set of functional and non-functional requirements for a Java style-checker plug-in for BlueJ to a set of system tests. In this assignment, the functional requirements play the role of the high-level artifact while the system tests play the role of the low-level artifact.

Once you log into RETRO a new project will automatically open with the candidate RTM loaded with the low and high level artifacts.

\section{Tracing}

Using the functionality provided to you by RETRO, complete the tracing task. You can use any functionality available to you through RETRO to determine the final (in your opinion) RTM. Please refer to the RETRO instructions sheet for more information.

Your goal is to validate the candidate links and mark the links you determine as valid as "Link". In addition you may search for links that the Candidate RTM did not include.

\section{Completing the Tracing Task}

Once you believe that you have correctly identified the Requirements Traceability Matrix for your task, click the "Submit" button. This will open up a window with detailed directions to submit your RTM and log files. These directions will contain the full path location of these files and where to send them.

Note: When submitting your final RTM ONLY links with "LINK" status will be included in the RTM. The final RTM will ignore default links.

\section{Post-Task Survey}

The experiment is considered completed after you have submitted the final RTM, log file and answered the post-experiment survey.

Once you submit the final RTM and the software use log to RETRO.researchers@gmail.com you will recieve a response from that email address with instructions and the URL for the survey. Once the post-survey has been completed your professor will be notified that you successfully completed the task.

If you have any questions during or after the experiment you may contact Alex Dekhtyar: dekhtyar@calpoly.edu, Jeff Holden: jholden@calpoly.edu, or Jane Hayes: hayes@cs.uky.edu

Thank you very much for your participation! 
Appendix D

\section{Experiment Instructions}


Instructions for working with RETRO

\section{RETRO}

RETRO, a.k.a., Requirements Tracing On Target is a software tool developed at the University of Kentucky and Cal Poly San Luis Obispo for the purpose of supporting the requirements tracing process. RETRO automates candidate link generation on tracing tasks.

\section{RETRO Instalation}

RETRO is a .NET program. At present RETRO has been tested only on the Windows operating system. Additionally, RETRO will only work on Windows machines that have the .NET framework installed. To our knowledge, the workstations at Cal Poly CS Labs and at the University of Kentucky CS Labs have the required .NET framework installed on them.

To download RETRO type the following URL in your web browser and download the zip file:

http://users.csc.calpoly.edu/ jholden/RETROV1.zip

After downloading the zip file extract the RETROV1 folder. Enter the RETROV1 folder and launch the application by running the Retro.exe file.

\section{Working With RETRO}

Login. The version of RETRO you will be working with requires user login. Each of you will be given the loginID and the password together with the experiment instructions.

Projects. Upon logging into the system a project containing your assignment will auto-load.

The project has two artifacts associated with it it: a low-level artifact (a collection of system tests) shown on the right side of the RETRO main window, and a high-level artifact (a collection of functional requirements), shown on the left side. Tracing takes place from the high-level artifact to the low-level artifact.

Once the artifacts are loaded the system will display several tabs for your use while tracing. The high-level artifact will be displayed on the All tab on the left, while the low-level artifact will be displayed on the By Recommendation tab on the right.

Each tab is separated into two areas. The top area is used to display the list of elements while the bottom tab is used to display the text of individual elements.

The high level document area has two tabs, All and By keyword. The low level document area has three tabs, All, By Keyword, and By recommendation. We briefly describe the functionality of each tab below.

All Tab. This tab displays all elements of the given (high/low level) artifact. For the high level document this is the main view. For the low level document it allows you to browse the entire low level elements whether they have been recommended.

By Keyword Tab. The Keyword tab is used to filter the elements of high and low level documents using simple keyword search. Any search string may be used and search strings may contain boolean expressions.

By Recommendation Tab. The Recommendation tab is the main tab for the low-level artifact. It is used to show the candidate RTM produced by the automated process and offered to you for validation. This tab will only show low-level elements which the system recommends for the selected high-level element, sorted by weight (higher weight indicates the system predicts the link is more likely to be true). 
Validating a link. All links provided to you in the assignment are candidate links and require you to validate them. To preform this task first select the high-level element you want to look at. After this the candidate low-level links are shown in the Recommendation tab of the low level artifact. Each candidate link has a status associated with it. The status is shown next to the low-level element ID. Three status values are available: Link, Not a Link and Default.

- Link. This status signifies the validated link. Assign this status to any link you believe to be correct. Only links with this status will be included in the final RTM you submit.

- Not a Link. This status signifies the candidate link is incorrect. Assign this status to any link you believe to be wrong.

- Default. This status is initially assigned by RETRO to ALL candidate links. Links marked as "Default" when submitting the final RTM will not be included in it. You may use the "Default" status as the indication that the candidate link has not been explicitly validated/rejected yet.

To change a link status select the desired status value from the list box and click off this row. The change will only be propagated after the click-off. Note, there is a status associated with high level elements as well. There is no need to modify this status for your task, although it is not forbidden.

Locating missing links. The candidate RTM provided to you for validation may include incorrect candidate links. It may also omit valid links. If you believe that your candidate RTM omits valid links it is your task to seek them out and include them in the final RTM you submit. To preform this task first locate the high-level element you want to look for missing low-level links for. After this select the All or keyword tabs from the low-level artifact window. You may now locate a low-level link that you determine should be a part of the final-RTM. To include this link in the RTM change the low-level status to Link and click off this row. The Link row will now be displayed in green.

Saving a project. At any time you may save your current trace progress by either clicking the save icon in the toolbar or going to File $\rightarrow$ Save option on the main menu, or using the Ctrl+S shortcut. After saving the RTM you may close the application.

When you restart RETRO (on the same computer) the next time, and log in using your login ID and password, the saved RTM will automatically reload.

Submitting final RTM. After you complete the tracing task, you need to submit the final RTM. To commence the submission process, click the Submit button on the toolbar. This will bring up the detailed submission instructions.

To submit the results of your work you need to locate two files in the RETRO folder. One file represents your final RTM in XML format (you can view the XML document matching the current state of your RTM at any time by using the Actions $\rightarrow$ Show XML option from the main menu of RETRO). The other file contains a succinct log of your activities with RETRO, recording the sequence of your validation decisions. The pop-up window will contain the exact names and paths to the files.

Once you see the instructions, open the File Explorer and locate the files in the RETROV1 folder. The actual submission is performed by sending these two files as attachment in an email to

retro. researchers@gmail.com

Put the following text in the subject line:

RETRO Experiment <University $><$ Course $>$

where <University> is the university in which you participated in the study (you may use abbreviations like "UK" or "Cal Poly") and <Course> is the course number through which you participated in the study.

NOTE: You may copy these instructions into a separate document if they are needed for future reference. 
Appendix E

\section{Pre-Study Survey}




\section{Pre-Study Questionnaire}

Automated Requirements Traceability: the Study of Human Analysts

0. What is your name?

1. What is your major? (circle one)
(A) Computer Science
(C) Computer Engineering
(B) Software Engineering
(D) Other:

2. What is your grade level? (circle one)
Freshman
Junior
Graduate
Sophomore
Senior

3. Which Software Engineering courses have you taken or are currently enrolled in? (circle all that apply or "none")

$\begin{array}{lll}\text { CSC-305 } & \text { CSC }-402 & \text { CSC-509 } \\ \text { CSC-307 } & \text { CSC }-405 & \text { NONE } \\ \text { CSC-308 } & \text { CSC-406 } & \\ \text { CSC-309 } & \text { CSC-508 }\end{array}$

4. In which Software Engineering courses did you build a requirements traceability matrix (RTM)? (circle all that apply or "none")

$\begin{array}{lll}\text { CSC-305 } & \text { CSC-402 } & \text { CSC-509 } \\ \text { CSC-307 } & \text { CSC-405 } & \text { NONE } \\ \text { CSC-308 } & \text { CSC-406 } & \\ \text { CSC-309 } & \text { CSC-508 }\end{array}$

5. What kind of industry experience do you have? (circle all that apply)

(A)Worked professionally before enrolling at Cal Poly.

(B) Work professionally while enrolled at Cal Poly

(C) Internship/Co-op

(D) None

6. Did you encounter traceability in your industry experience?

Yes

No

7. How comfortable do you feel about your ability to perform requirements tracing? (circle one)

$\begin{array}{lll}\text { Very uncomfortable } & \begin{array}{l}\text { Neither comfortable or } \\ \text { uncomfortable }\end{array} & \text { Very comfortable }\end{array}$

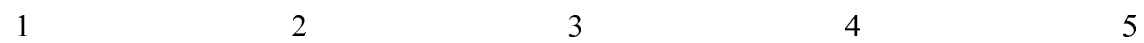

8. Where did you encounter requirements traceability for the first time?

(A) Have not encountered before

(B) Encountered in a CSC/SE college course (Cal Poly or elsewhere)

(C) Encountered while on an industry internship or co-op

(D) Encountered while employed in the industry (full-time or part-time) 
9. When did you encounter requirements traceability for the first time?
(A) Have not encountered before
(B) Between Fall 2009 and Spring 2010
(C) Some time between Fall 2006 and Summer 2009
(D) Some time earlier than Fall 2006

10. How many times have you performed requirements tracing since then?
(A) Never
(B) Once
(C) Two to four times
(D) Five or more times

11. What did you use when performing requirements tracing? (circle all that apply)
(A) Did not perform tracing tasks
(B) Used hard copies of artifacts to perform tracing
(C) Used soft copies of artifacts to perform tracing
(D) Used word processor to perform tracing
(E) Used a spreadsheet or a database to maintain RTM
(F) Used a CASE tool to assist in tracing
(G) Used some other tool/technique/method to perform tracing:

Specify:

\section{Have you ever encountered the CheckStyle Data Set?}
(A) No
(B) I have heard of it but never seen it
(C) I attended a previous thesis defense where the data set was discussed.
(D) I participated in a previous study that used it

13. Is your native language English?

YES

NO

14. What else should we know about your experience with traceability? 
Appendix F

\section{Post-Study Survey}




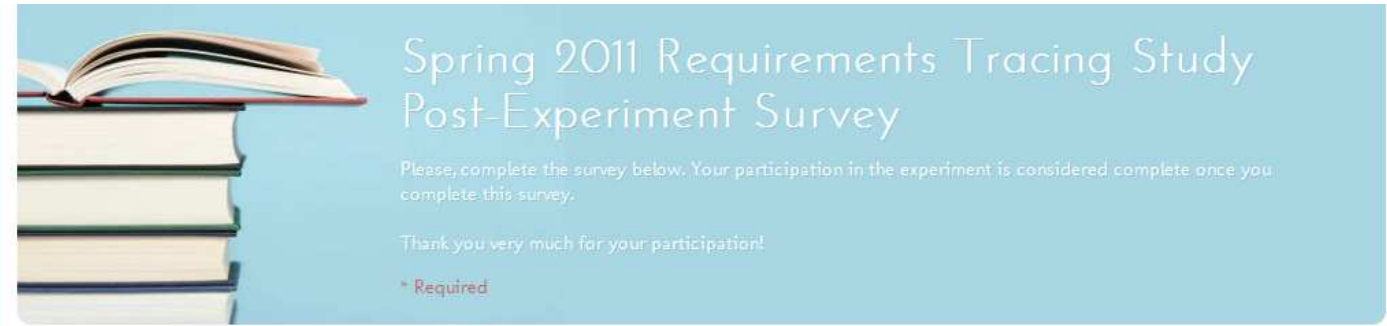

User Id *

Please, enter your RETRO ("userNN" or "userNNN")

I

Which school did you perform the tracing task at? *

Cal Poly

(1) University of Kentucky

- DePaul University

Between the completion of the in-lab training session and the beginning of the experiment, did you spend any time using RETRO? *

select one

(1) Yes

№

How much time did you spend using RETRO?

If you answered "Yes" to the previous question, please provide the answer.

(1) Less than 30 mins

$30-60$ mins

(1- 2 hours

$2+$ hours 
In the task you performed for this study. how often did you perform the following tasks :

Select N/A if the UI you worked with did not provide the appropriate functionality.

\begin{tabular}{|c|c|c|c|c|c|}
\hline & Never & Rarely & So metimes & Frequently & $\mathrm{N} / \mathrm{A}$ \\
\hline Se arched for o m itted links & $\bigcirc$ & 0 & 0 & $\bigcirc$ & $\bigcirc$ \\
\hline Rejected offered candidate links & O & 0 & 0 & O & ○ \\
\hline $\begin{array}{r}\text { Seare hed requirem ents by } \\
\text { keywo rd }\end{array}$ & 0 & 0 & ○ & 0 & O \\
\hline Searched test cases by keyword & 0 & 0 & 0 & $\bigcirc$ & 0 \\
\hline $\begin{array}{r}\text { used 'Actions } \rightarrow \text { Trace All"action } \\
\text { from the main menu }\end{array}$ & ○ & ○ & 0 & $\bigcirc$ & 0 \\
\hline $\begin{array}{l}\text { used "Actions }->\text { Trace Selection" } \\
\text { action from the main menu }\end{array}$ & 0 & $\bigcirc$ & 0 & $\bigcirc$ & 0 \\
\hline $\begin{array}{r}\text { Used View Keywo rds" button for } \\
\text { requirem ents }\end{array}$ & 0 & 0 & 0 & $\bigcirc$ & $\bigcirc$ \\
\hline $\begin{array}{r}\text { Used View Keywords" button for } \\
\text { test cases }\end{array}$ & $\bigcirc$ & 0 & $\bigcirc$ & $\bigcirc$ & ○ \\
\hline
\end{tabular}

Rate the following statements on a scale from 1 to 5 indicating how strongly you agree of disagree with the statement.

\begin{tabular}{|c|c|c|c|c|c|}
\hline & $\begin{array}{l}\text { E: Strongly } \\
\text { Disagree }\end{array}$ & 2: Disagree & 3: Neutral & 4: Agree & 5: Stro ngly Agree \\
\hline $\begin{array}{l}\text { (A) The training session provided } \\
\text { me with sufficient information to } \\
\text { complete my task }\end{array}$ & 0 & $\bigcirc$ & $\bigcirc$ & $\bigcirc$ & 0 \\
\hline $\begin{array}{l}\text { (B) RETRO reduces the effort } \\
\text { required to trace require m ents }\end{array}$ & 0 & 0 & $\bigcirc$ & 0 & 0 \\
\hline $\begin{array}{l}\text { (C) RETRO reduces the time } \\
\text { required to trace require ments }\end{array}$ & 0 & 0 & $\bigcirc$ & $\bigcirc$ & $\bigcirc$ \\
\hline $\begin{array}{r}\text { (D) Tracing requirem ents with } \\
\text { RETRO is easy }\end{array}$ & ○ & $\bigcirc$ & ○ & $\bigcirc$ & $\bigcirc$ \\
\hline $\begin{array}{l}\text { (E) My classes prepared me to } \\
\text { perfo rm the task that I was asked } \\
\text { to perfo rm }\end{array}$ & 0 & 0 & 0 & 0 & 0 \\
\hline $\begin{array}{l}\text { (F) My professio nal experience } \\
\text { prepared me to perfo rm the task } \\
\text { that I was asked to perform }\end{array}$ & $\bigcirc$ & 0 & ○ & 0 & ○ \\
\hline $\begin{array}{l}\text { (G) I was prepared to perfo rm the } \\
\text { task that I was asked to perfo rm }\end{array}$ & 0 & 0 & 0 & 0 & 0 \\
\hline
\end{tabular}

How com fortable do you feel about your ability to perform requirem ents tracing?

$\begin{array}{llllllllllllllll}1 & 2 & 3 & 4 & 5\end{array}$

very uncomfortable $\bigcirc \bigcirc \bigcirc \bigcirc$ Very comfortable

Would you be more com fortable tracing by hand or using a so ftware tool?

By hand

Using so ftware

In what ways do you think RETRO $\mathrm{c}$ an be im proved!

please, provide any suggestions

please, provide any suggestions

Is there anything else you would like to tell us regarding your experience using RETRO to perform requirements tracing? please, expand on any of your answers above

,

Have you participated in tracing experiments prior to this one?

please, anwer "participated, but did not com plete" if you received a RETRO userID in a prior experiment, but elected to not complete the task.

Participated, and completed the task

(1) Participated, but did not complete the task

Did not participate 


\section{Appendix G}

\section{Training Dataset}

\section{G.1 Functional Requirements}

\section{FM-1}

Upon its start, the program shall print information about its purpose

(moon landing game) and author:

********* Moon Landing Program v. $1.0 * * * * * * * * * * * * * * * * * *$

Written by: Alexander Dekhtyar

\section{FM-2}

After that the main loop of the program starts. At the beginning of each

loop, the program shall display a text menu with three choices and prompt

the user to make a selection. The menu choices are 1. Play Game,

2. Instructions, 3. Quit:

Please select an action:

1. Play Game

2. Instructions

3. Quit

Your choice : \>

(Note, there is an empty line between the Written by: line of the
output and the Please select an action: line. Note also that : >

is the prompt used in the program.) 


\section{FM-3}

Your program shall accept user input, indicating the desired action. Acceptable user inputs are integers $1,2,3$. If any other integer number is enered, the program shall simply output the main menu and the prompt

again:

********** Moon Landing Program v. $1.0 * * * * * * * * * * * * * * * * * *$

Written by: Alexander Dekhtyar

Please select an action:

1. Play Game

2. Instructions

3. Quit

Your choice : $>8$

Please select an action:

1. Play Game

2. Instructions

3. Quit

Your choice : >>

Your program is not responsible for handling any other type of input (e.g.,

if a character or a string has been entered instead of an integer).

\section{FM-4}

If the user chooses Quit, i.e., enters 3 the program shall print a good-

bye message and exit.

Please select an action:

1. Play Game

2. Instructions

3. Quit

Your choice : >>3

Bye ! Thanks for playing Moon Landing

\section{FM-5}

If the user chooses Instructions, i.e., enters 2 the instructions on

how to play the game shall be printed After that the program shall loop

back to the menu. The text of the instructions is as follows:

Please select an action:

1. Play Game

2. Instructions

3. Quit

Your choice : $>2$

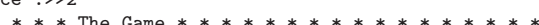

You are to land a spaceship on the moon.

You control the speed of your spaceship by telling it

how much fuel has to be burned every second.

5 units of fuel cancel the Moon gravity, more - will deccelerate your ship

Do not run out of fuel.

To land successfully your velocity should not exceed 10 feet/sec

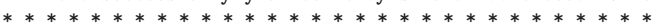

Please select an action:

1. Play Game

2. Instructions

3. Qui

Your choice : > 


\section{FM-6}

If the user chooses Play Game, i.e., enters 1, the game shall com-

mence. See requirements below for the in-game specifications. After the

game is over, the program shall loop back to the menu.

\section{FM-7-1}

During the game initialization stage, the program shall set the initial values

for the distance to the Moon, amount of fuel left and ship velocity. These

parameters were specified above.

\section{FM-7-2-1}

At the beginning of each game loop iteration, your program shall

display current status of the game. Current status includes

i. time elapsed since the beginning fo the landing (in seconds)

ii. distance left to the Moon (in feet);

iii. amount of fuel left (units)

iv. current velocity (feet/sec)

\section{FM-7-2-2}

After the status is displayed, the program shall prompt the user to enter amount of fuel to be burned (injected) over the next second.

The program shall read the amount of fuel (an int value).

The amount of fuel entered by the user shall be tested: it shall not

be negative and it cannot exceed amount of fuel left. The prompt for

the amount of fuel to burn shall be repeated and a new value read

until a valid value had been entered.

\section{FM-7-2-3}

Once a valid amount of fuel is read the program compute the new

velocity of the ship and the new distance to the moon. Use the

formulas above to compute this. 


\section{G.2 System Tests}

\section{TC-1}

\section{Steps}

1. Start Moon Lander.

Output

Please select an action:

1. Play Game

2. Instructions

3. Quit

Your choice :>>

\section{TC-2}

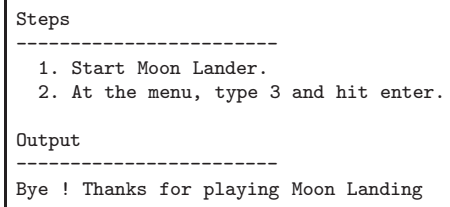

\section{TC-3}

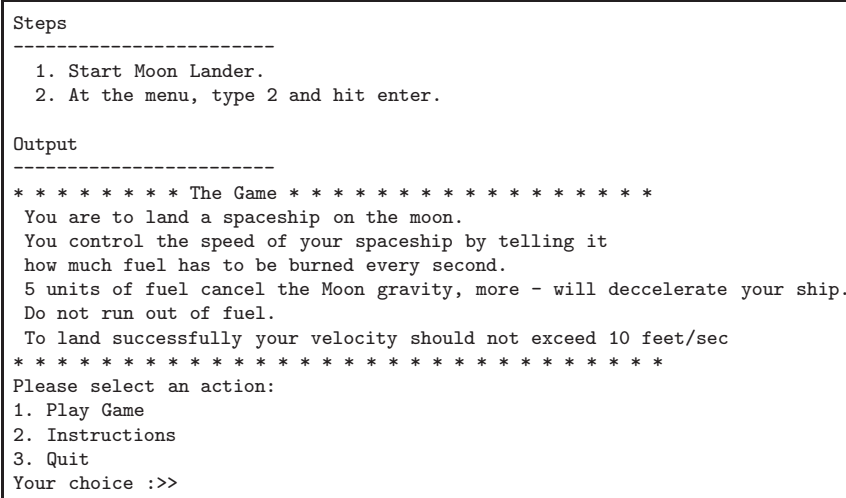

\section{TC-4}

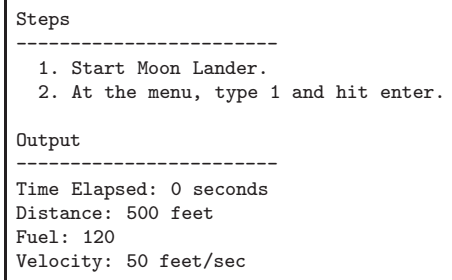




\section{TC-5}

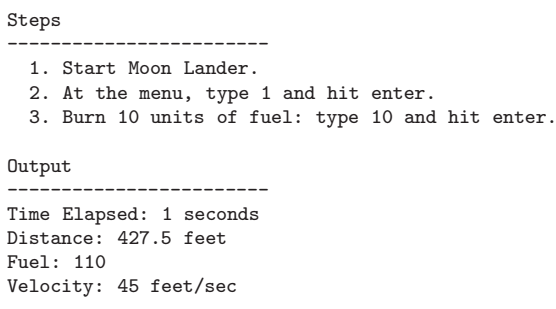

Time Elapsed: 1 seconds

Distance: 427.5 feet

Fuel: 110

Velocity: $45 \mathrm{feet} / \mathrm{sec}$ 


\title{
Appendix $\mathrm{H}$
}

\section{Experimental Dataset}

\author{
H.1 Requirements
}

2.1.1

Formats source code in the BlueJ editor window on demand (does not format on the fly).

2.1.2

The user can edit the Style Preferences through the Style Preference GUI interface.

2.1.3

The user may specify or use default Style Preferences. 


\section{1 .4}

Presents the user the option to choose from preset Style Preferences (such as the GNU or JAVA styles) as well as user's Style Preferences.

\section{1 .5}

Immediately after ChangeStyle formats the code, the user may restore the file to its state right before the format occurred.

\subsection{6}

Support all standard BlueJ class types \{Class, Abstract Class, Interface, Applet, Unit Test, Enum\}.

\subsection{7}

Available External Documentation (user manual)

\subsubsection{0}

Has the ability to format multiple files at the same time (i.e. all source code in a package.) Also have the ability to format just one file at a time.

\section{1 .12}

ChangeStyle will only format compliable code

\section{1 .13}

ChangeStyle only formats files ending in '.java'. 


\section{1 .14}

Every panel in the settings dialog will have a personal help link linking to the corresponding section in the Jalopy User Manual, if the section is available.

\section{1 .15}

Customized Conventions ChangeStyle will allow the user to modify predefined conventions and save them as customized conventions that will appear in the conventions drop down box along with the original predefined conventions.

\section{1 .16}

Batch Undo ChangeStyle implement Batch Undo, which presents the user with a dialog with a selectable list of files which can be undone, while displaying files which cannot be undone in grey.

\subsubsection{7}

Editor Update Changes made by ChangeStyle's formatting are visible immediately in the editor window, and when the Undo button

is selected in the editor window, the editor window shows the undone code.

\section{1 .18}

Import/Export The import and export convention functionality for ChangeStyle is located in the Preferences Panel and is designed based on the approved UI prototype.

\section{1 .19}

Headers/Footers ChangeStyle allows the user to replace Headers and Footers containing some user-specified text in the corresponding panels and offers a help link to a new section in the User Manual describing Headers and Footers in more detail.

\section{1 .20}

Status Panel Every time a class or project is formatted by ChangeStyle, the 'status panel' at the bottom of BlueJ's window displays "Formatting... Done." When the undo feature is selected, the window will display "Undo Formatting... Done" 


\section{1 .21}

Preference Panel When the users clicks, the "Edit..." button, no matter how many times, the Preferences panel only appears once.

\subsection{1}

Minimum: 64MB main memory, Pentium II processor or equivalent Recommended: 128MB main memory, 400MHz Pentium III processor or above

\subsection{2}

ChangeStyle does not take more than 10 seconds to format 1000 lines of code.

\subsection{3}

J2SE 1.6.0 (Java 2 SDK version 1.6.0) or newer must be installed.

\section{0 .4}

ChangeStyle runs on BlueJ 2.2 or newer

\section{0 .5}

ChangeStyle runs on Windows XP (SP2) and Mac OSX and Linux

\subsection{6}

ChangeStyle formats compiled code according to Jalopy's formatting convention standards 
3.0 .9

The user can add and modify Style Preferences through the UI Portability

\section{0 .10}

ChangeStyle is an extension to BlueJ which only runs on the Java Runtime Environment Reliability

\section{0 .11}

ChangeStyle's Mean Time Between Defects (MTBD) is greater than 30 formats.

\section{0 .12}

ChangeStyle does not format on uncompilable .java files.

\section{0 .14}

ChangeStyle does not add any new expressions to the formatted code.

\section{0 .16}

A Computer Science student can download and install ChangeStyle in less than 30 minutes on a computer that has BlueJ already installed.

\section{0 .17}

A Computer Science student can format a .java file in BlueJ that has ChangeStyle already installed in less than 5 minutes. 


\section{0 .18}

Formatting a file using the currently set Style Convention takes less than 4 clicks. 


\section{H.2 System Tests}

\section{TC-1}

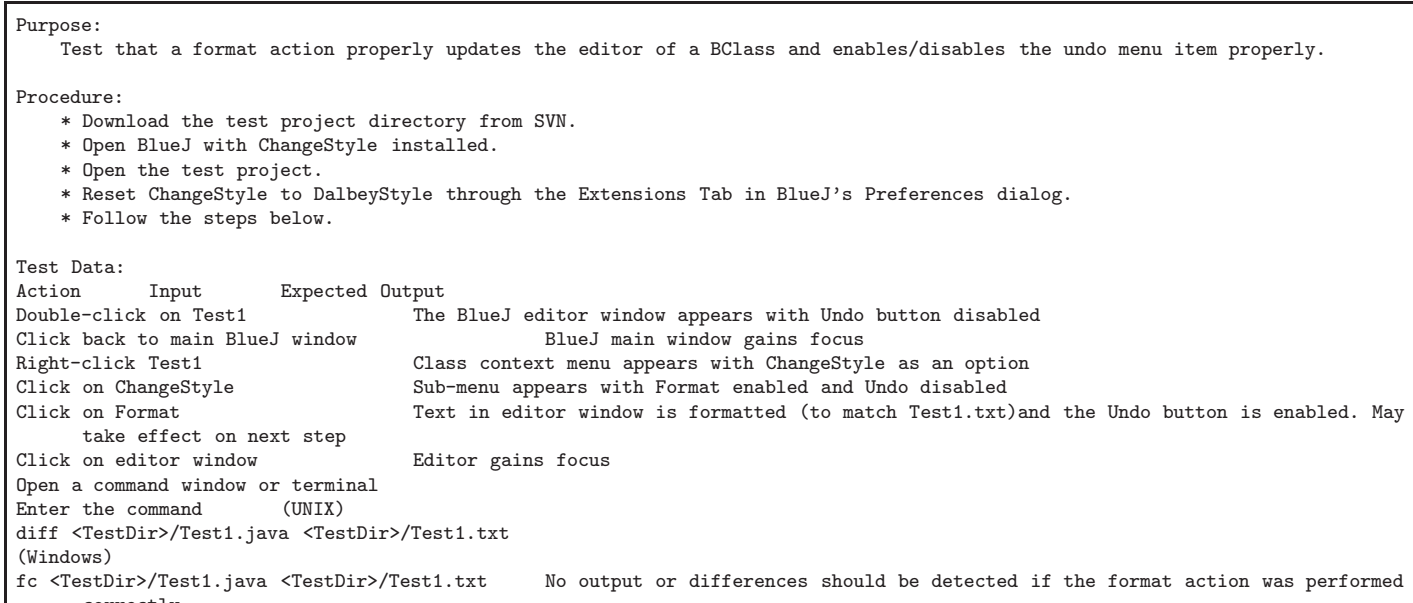

\section{TC-2}

Purpose:

To show how the user can change between different Style Preferences as well as user's Style Preferences.

Procedure:

* Open BlueJ with ChangeStyle installed.

* Create a new project.

* Create a new Class

* Follow the steps below.

Test Data:

Action Input Expected Output

In main BlueJ window, click the 'Tools' located in the menu across the top of the screen A pull-down menu appears

Select and click 'Preferences...' A pop up window appears giving information about preferences
Find and click on the 'Extensions' tab
The tab should change and you should see different detail sections for

The tab should change and you should see different detail sections

Under the ChangeStyle detatil section, click the button labeled 'Edit...' 'Preview' pop-up window should appear

Looking over the 'Custom Settings' pane, you can find under the 'Braces' default option, 'Styles' option, different preset styles to choose from or you can go through each option and create your own style preferences selecting ' $\mathrm{Ok}$ ' when a format is invoked, the code should be formatted according to the chosen style 


\section{TC-3}

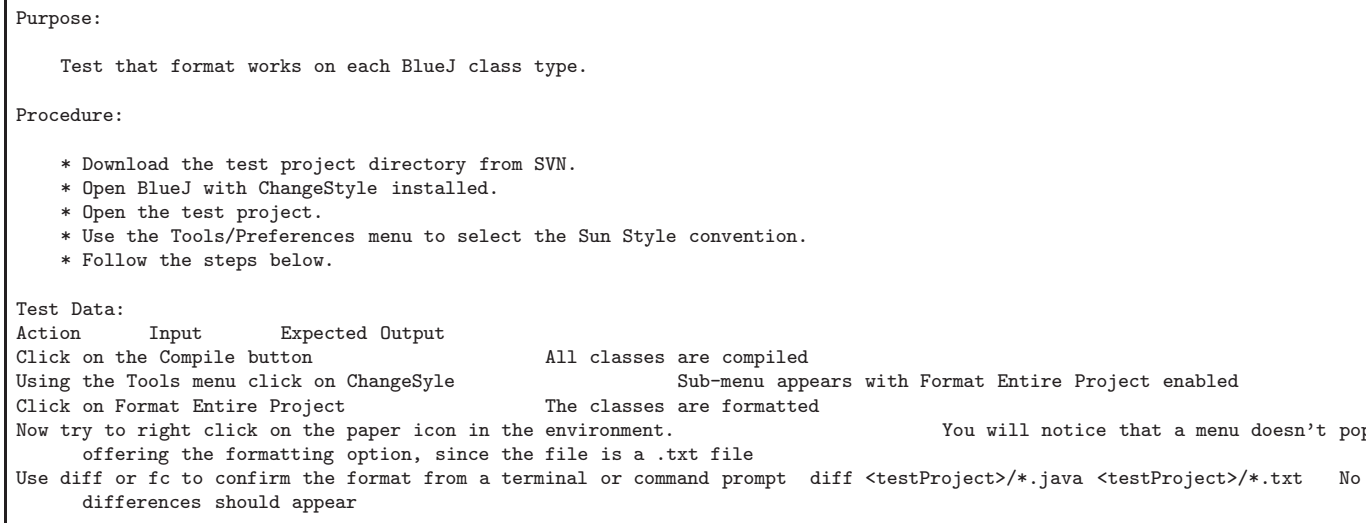

\section{TC-4}

Purpose:

Show where to locate available external documentation.

Procedure:

* Open BlueJ with ChangeStyle installed.

* Create a new project.

* Reset Jalopy to its default settings through the Extensions Tab in BlueJ's Preferences dialog.

* Follow the steps below.

Test Data:

Action Input Expected Output

In main BlueJ window, click the help tab at the top of the screen A pull-down menu appears

Select and click 'Installed Extensions' a the top of the screen A pop up window appears giving information about all the installed extensions

Click on the bubble with the question mark inside of it (next to the status column of the ChangeStyle installed extension) A pop up window should occur giving Extension Details

Click on the link provided next to the 'More Information At:' label A web browser should pop up, open to Luna's Home Page

Find and click on the 'User Manual' link pertaining to your version of the ChangeStyle extension the User Manual 


\section{TC-5}

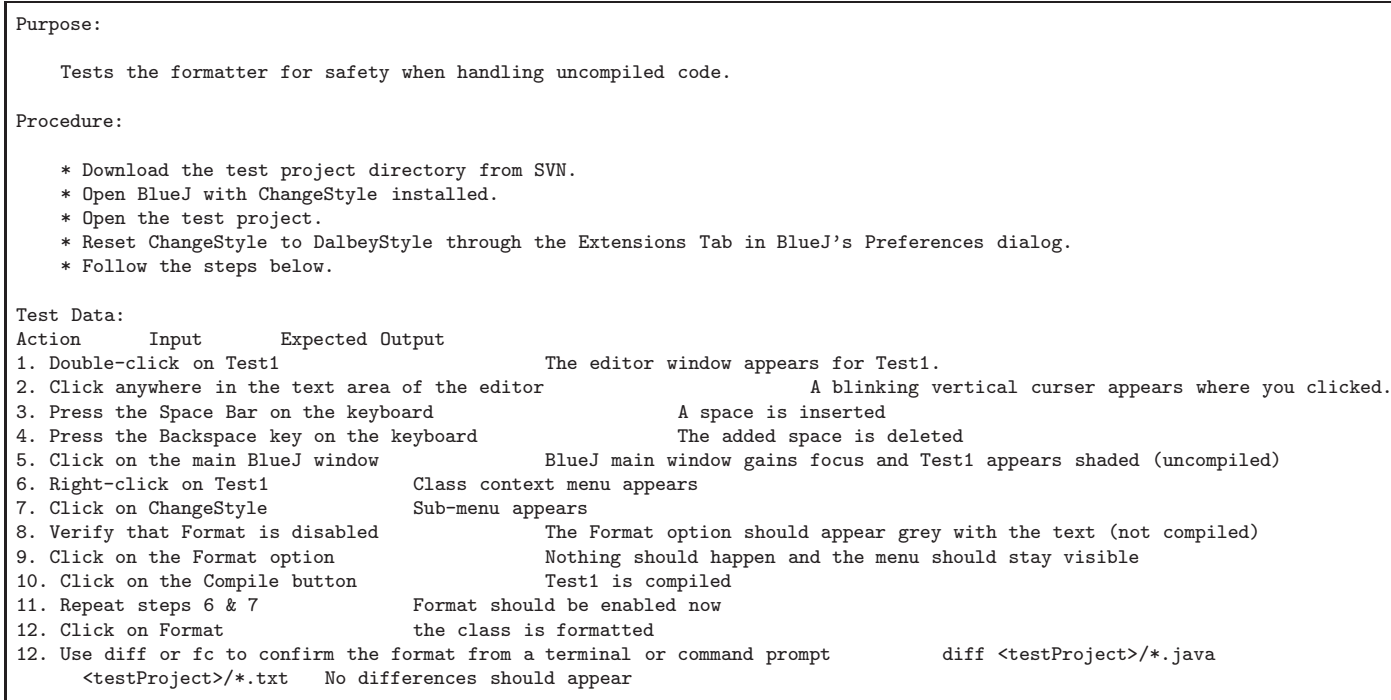

\section{TC-6}

Purpose:

Verify that ChangeStyle will operate on a computer meeting the system specifications.

Procedure:

* Look up the computers memory and the processor information.

* Follow the steps below:

Test Data:

Action Input Expected Output

Look up main memory Minium 64MB of memory

Look up processor Pentium II or equivalent

Verify both processor and main memory meet or exceed minimum requirements Pentium II or equivalent, 64MB of memory
BlueJ

Verify ChangeStyle is installed: Help->Installed Extensions ChangeStyle should be displayed under Installed Extensions

\section{TC-7}

Purpose:

Verify that ChangeStyle takes 1 seconds or less to format 100 lines of code.

Procedure:

* Format a class that has about than 100 lines of code. Since comments can be formatted as well they are included.

* Follow the steps below:

Test Data:

Action Input Expected Output

Start BlueJ BlueJ window open

Open testcase07.java Class should appear in BlueJ window

Open Timer Timer should be at 00:00:00

Start Timer and Format testCase07.java Confirm button should appear

Stop Timer when comfirm button apears $\quad$ Timer should disply less than 00:00:01.00 or 1 second. 


\section{TC-8}

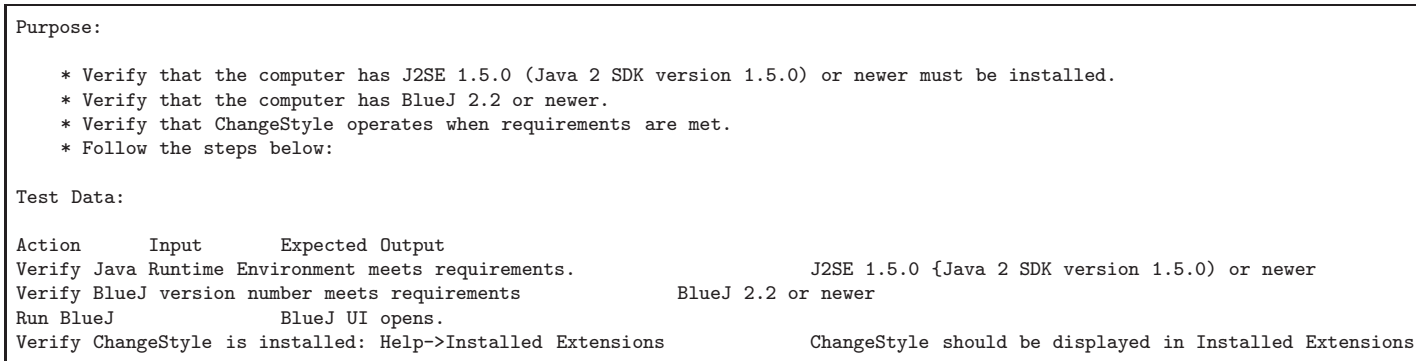

\section{TC-9}

Purpose:

* Checks to see if ChangeStyle was downloaded and works for Windows XP (SP2), Mac OSX and Red Hat Linux.

Procedure:

Follow the steps below:

Test Data:

Action Input Expected Output

Download ChangeStyle as a BlueJ extension

Open BlueJ Class should appear in BlueJ window

Open Help Help window will open

Click on Installed Extensions BlueJ:Installed Extensions windo will open

Window will say loaded ChangeStyle

Format testCase09.java Confirm window will open, saying class has been formatted.

Do this for all System, Windows XP (SP2), Mac OSX and Red Hat Linux

\section{TC-10}

Purpose:

To verify that ChangeStyle requires that a Java Runtime Environment be installed to run.

Procedure:

* Verify that the computer you are working on does not have a Java Runtime Environment installed. If there is one installed, then uninstall it.

* Follow the steps below.

Test Data:

Action Input Expected Output

Attempt to download and install BlueJ using the instructions here:

http://www.bluej.org/download/download.html BlueJ should not be able to install. 


\section{TC-11}

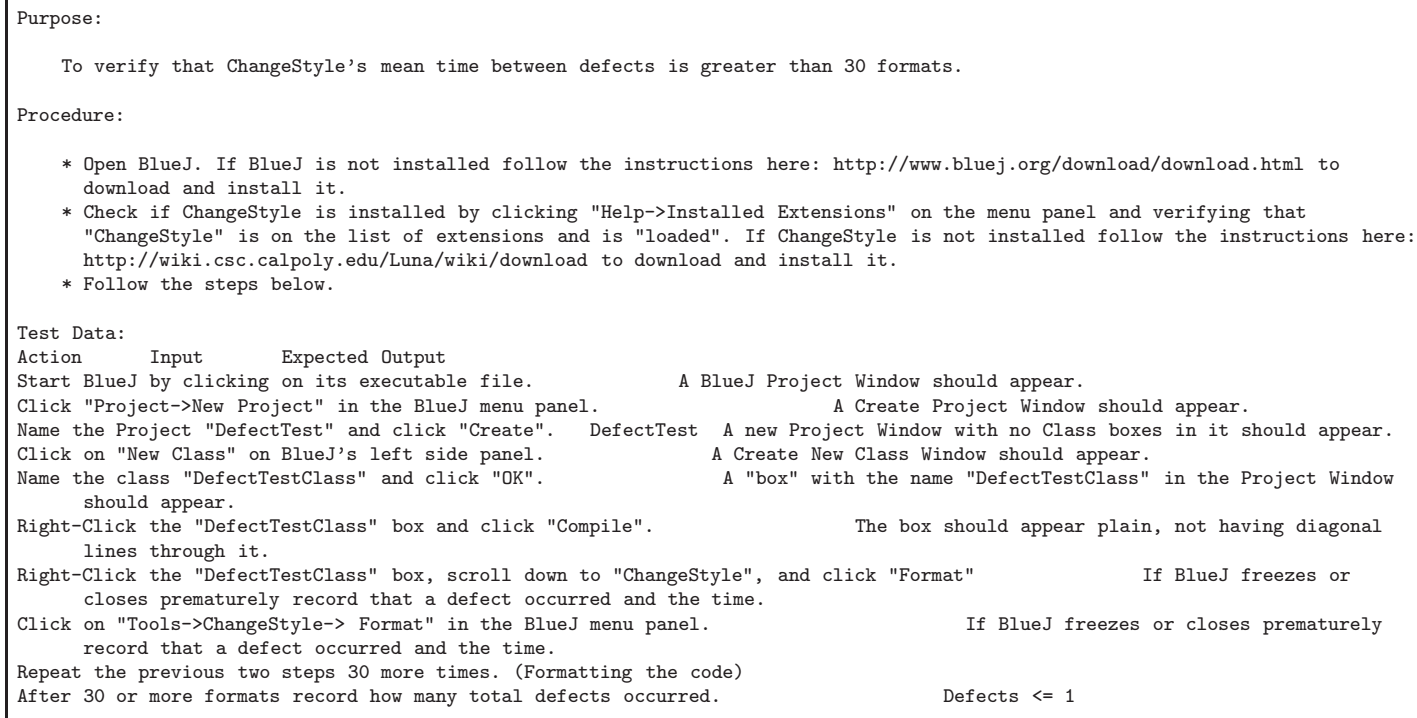

* Open BlueJ. If BlueJ is not installed follow the instructions here: http://www.bluej.org/download/download.html to download and install it.

* Check if ChangeStyle is installed by clicking "Help->Installed Extensions" on the menu panel and verifying that "ChangeStyle" is on the list of extensions and is "loaded". If ChangeStyle is not installed follow the instructions here: http://wiki.csc.calpoly.edu/Luna/wiki/download to download and install it.

* Follow the steps below.

Test Data:

Action Input Expected Output

Start BlueJ by clicking on its executable file. A BlueJ Project Window should appear.

Click "Project->New Project" in the BlueJ menu panel. A Create Project Window should appear.

Name the Project "DefectTest" and click "Create". DefectTest A new Project Window with no Class boxes in it should appear. Click on "New Class" on BlueJ's left side panel. A Create New Class Window should appear.

Name the class "DefectTestClass" and click "OK". A "box" with the name "DefectTestClass" in the Project Window should appear.

Right-Click the "DefectTestClass" box and click "Compile". The box should appear plain, not having diagonal lines through it.

Right-Click the "DefectTestClass" box, scroll down to "ChangeStyle", and click "Format" If BlueJ freezes or closes prematurely record that a defect occurred and the time.

Click on "Tools->ChangeStyle-> Format" in the BlueJ menu panel. If BlueJ freezes or closes prematurely record that a defect occurred and the time.

Repeat the previous two steps 30 more times. (Formatting the code)

After 30 or more formats record how many total defects occurred.

Defects $<=1$

\section{TC-12}

Purpose:

To verify that ChangeStyle does not format on uncompilable .java files.

Procedure:

* Open BlueJ. If BlueJ is not installed follow the instructions here: http://www.bluej.org/download/download.html to download and install it.

* Check if ChangeStyle is installed by clicking "Help->Installed Extensions" on the menu panel and verifying that

"ChangeStyle" is on the list of extensions and is "loaded". If ChangeStyle is not installed follow the instructions here:

http://wiki.csc.calpoly.edu/Luna/wiki/download to download and install it.

* Follow the steps below.

Test Data:

$\begin{array}{lll}\text { Action } & \text { Input } & \text { Expected Output } \\ \text { Start BlueJ by clicking on its executable file. A BlueJ Project Window should appear. }\end{array}$

Click "Project->New Project" in the BlueJ menu panel. A Create Project Window should appear.

Name the Project "FormatTest" and click "Create". FormatTest A new Project Window with no Class boxes in it should appear.

Click on "New Class" on BlueJ's left side panel. A Create New Class Window should appear.

Name the class "FormatTestClass" and click "OK". A "box" with the name "FormatTestClass" in the Project Window should appear.

Double click on the "FormatTestClass" box. A Class Editor Window should appear.

Type "not valid code" as the first line of the Class and close the Editor Window. not valid code The Class Editor Window should disappear.

Right-Click the "FormatTestClass" box and scroll down to "ChangeStyle". (not compiled)" should appear.

Click on "Tools->ChangeStyle" in the BlueJ menu panel. A grayed-out button that says "Format (not compiled)" should appear. 


\section{TC-13}

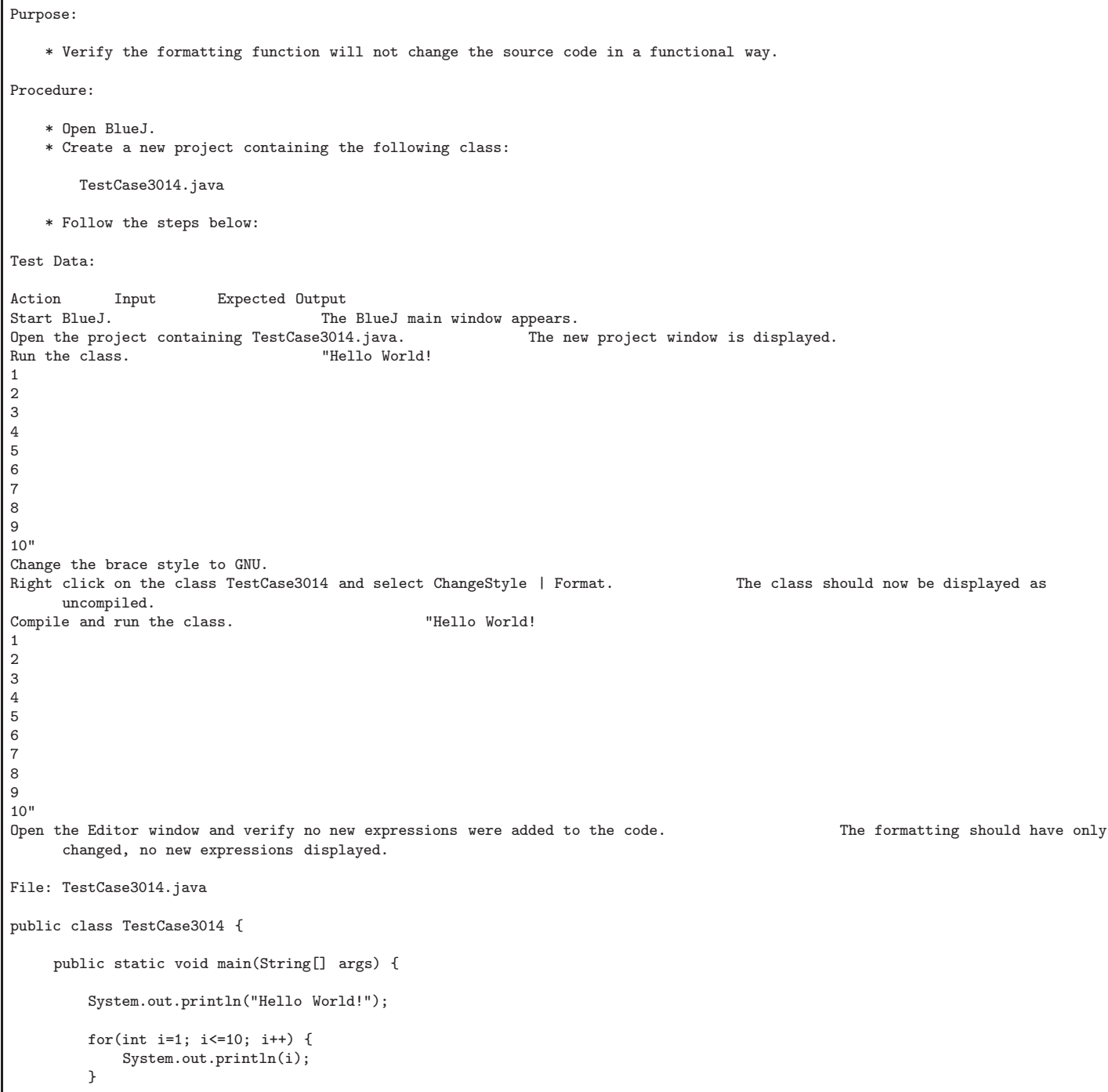




\section{TC-14}

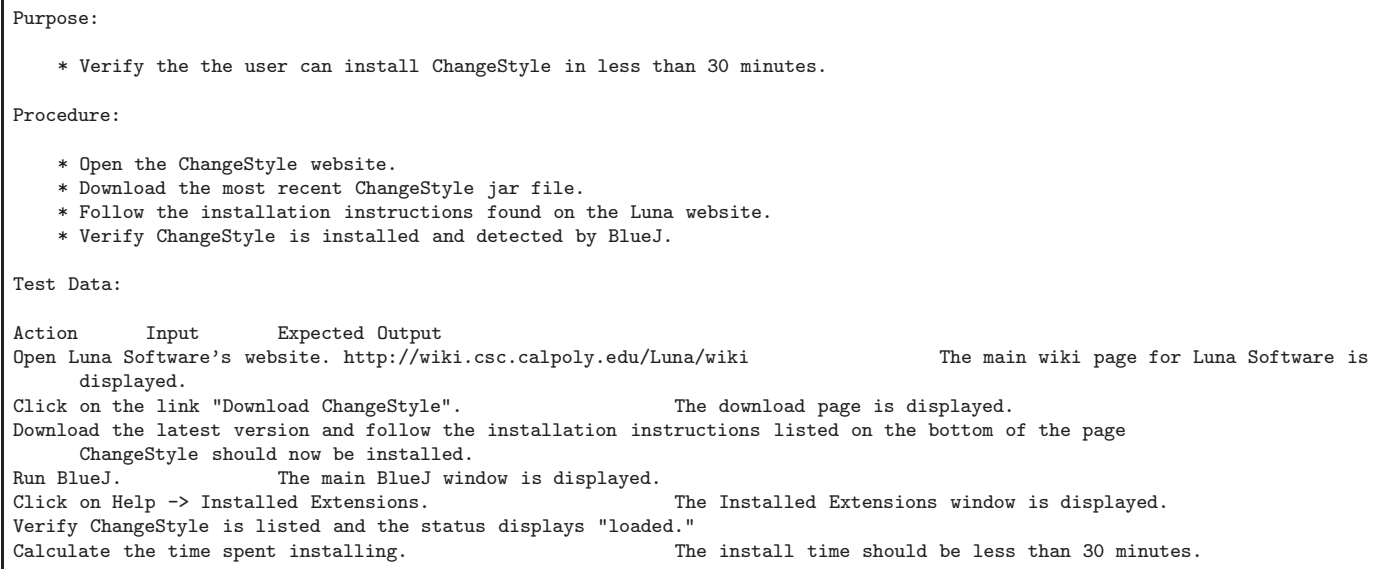

\section{TC-15}

Purpose:

* Verify the user can format the code in under 5 minutes.

* Verify the user can format in as little as two clicks.

Procedure:

* Open BlueJ.

* Create a new project containing the following class:

TestCase3017.java

* Follow the steps below:

Test Data:

Action Input Expected Output

Start BlueJ. The BlueJ main window appears.

Open the project containing TestCase3017.java. The new project window is displayed.

Right click on the class TestCase 3017 and select ChangeStyle I Format. The class is now formatted and should be displayed as uncompiled.

Verify the procedure took less than 5 minutes.

File: TestCase3017.java

public class ! TestCase3014

public static void main(String[] args)

System.out.println("Hello World!");

for (int $i=0 ; i<=10 ; i++$ ) \{

\}

System. out.println(i); 


\section{TC-17}

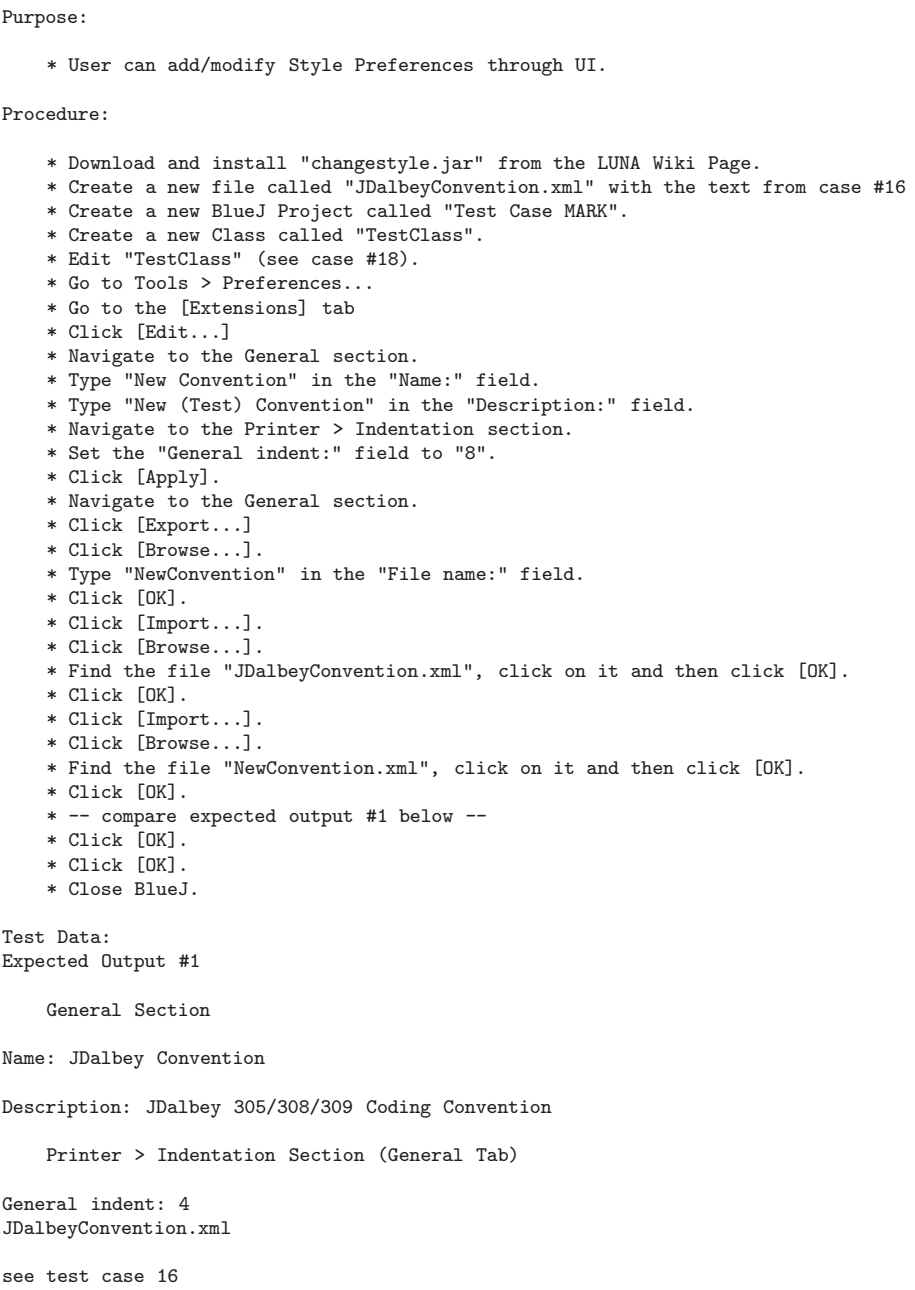

Name: JDalbey Convention

Description: JDalbey 305/308/309 Coding Convention

Printer > Indentation Section (General Tab)

General indent: 4

JDalbeyConvention.xml

see test case 16 


\section{TC-18}

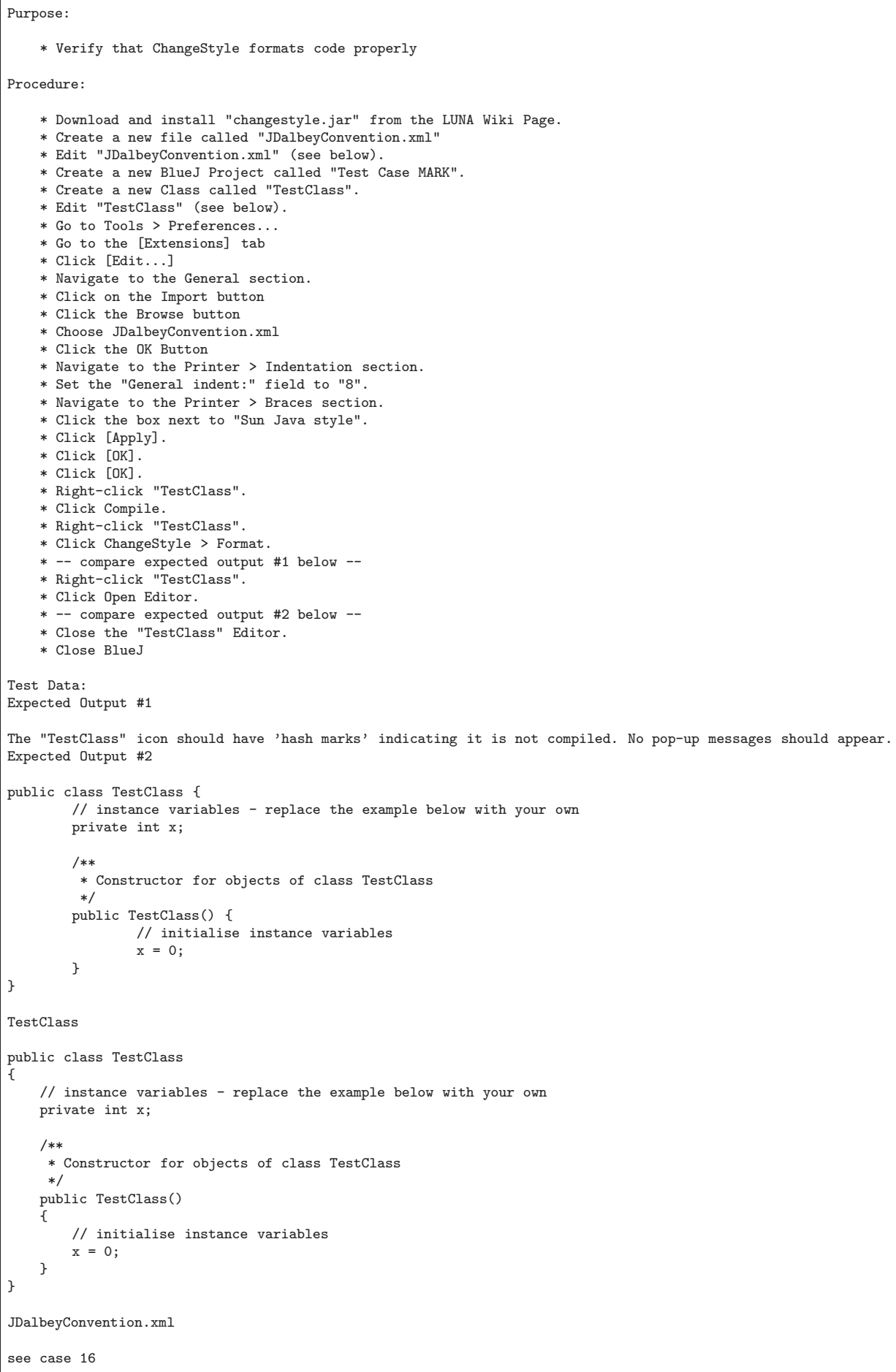




\section{Appendix I}

\section{HIPAA Requirements}

\section{I.1 Requirements}

\section{$\mathrm{AC}$}

Access Control. Implement technical policies and procedures for electronic information

systems that maintain electronic protected health information to allow access only to

those persons or software programs that have been granted access rights as specified

in ? $164.308(a)(4)$

$\mathrm{AL}$

Automatic logoff. Implement electronic procedures that terminate an electronic session

after a predetermined time of inactivity.

\section{AUD}

Audit Controls. Implement hardware, software, and/or procedural mechanisms that record

and examine activity in information systems that contain or use electronic protected

health information. 


\section{EAP}

Emergency access procedure. Establish (and implement as needed) procedures for obtaining

necessary electronic protected health information during an emergency

\section{IC}

Integrity controls. Implement security measures to ensure that electronically transmitted

electronic protected health information is not improperly modified without detection

until disposed of.

\section{PA}

Person or entity authentication. Implement procedures to verify that a person or entity

seeking access to electronic protected health information is the one claimed.

\section{SED}

Encryption. Implement a mechanism to encrypt electronic protected health information

whenever deemed appropriate.

\section{TED}

Encryption and decryption. Implement a mechanism to encrypt and decrypt

electronic protected health information

\section{TS}

Transmission security. Implement technical security measures to guard against

unauthorized access to electronic protected health information that is being

transmitted over an electronic communications network.

\section{UUI}

Unique user identification. Assign a unique name and/or number for

identifying and tracking user identity. 